\title{
On-Line Analysis of Exhaled Breath
}

\section{Review Article}

Author(s):

Bruderer, Tobias (D); Gaisl, Thomas; Gaugg, Martin T.; Nowak, Nora; Streckenbach, Bettina; Müller, Simona Olivia (D); Moeller, Alexander; Kohler, Malcolm; Zenobi, Renato (iD

Publication date:

2019-10-09

Permanent link:

https://doi.org/10.3929/ethz-b-000372767

Rights / license:

In Copyright - Non-Commercial Use Permitted

Originally published in:

Chemical Reviews 119(19), https://doi.org/10.1021/acs.chemrev.9b00005 


\title{
On-line analysis of exhaled breath
}

Authors: Tobias Bruderer ${ }^{1,2+}$, Thomas Gaisl ${ }^{3,4,5+}$, Martin T. Gaugg ${ }^{1}$, Nora Nowak ${ }^{1}$, Bettina Streckenbach ${ }^{1}$, Simona Müller ${ }^{1}$, Alexander Moeller ${ }^{2}$, Malcolm Kohler ${ }^{3,4,5}$, Renato Zenobi ${ }^{1 *}$

\author{
Affiliations: \\ ${ }^{1}$ Department of Chemistry and Applied Biosciences, Federal Institute of Technology, 8093 Zurich, \\ Switzerland \\ ${ }^{2}$ Division of Respiratory Medicine, University Children's Hospital Zurich and Children's Research Center \\ Zurich, 8032 Zurich, Switzerland \\ ${ }^{3}$ Department of Pulmonology, University Hospital Zurich, 8091 Zurich, Switzerland \\ ${ }^{4}$ Center for Integrative Human Physiology, University of Zurich, 8091 Zurich, Switzerland \\ ${ }^{5}$ Zurich Center for Interdisciplinary Sleep Research, University of 8091 Zurich, Zurich, Switzerland \\ ${ }^{+}$Shared first authorship, *Corresponding author, zenobi@org.chem.ethz.ch
}

\begin{abstract}
On-line analysis of exhaled breath offers insight into a person's metabolism without the need for sample preparation or sample collection. Due to its non-invasive nature and the possibility to sample continuously, the analysis of breath has great clinical potential. The unique features of this technology make it an attractive candidate for applications in medicine, beyond the task of diagnosis. We review the current methodologies for on-line breath analysis, discuss current and future applications, and critically evaluate challenges and pitfalls such as the need for standardization. Special emphasis is given to use of the technology in diagnosing respiratory diseases, potential niche applications, and the promise of breath analysis for personalized medicine. The analytical methodologies used range from very small and low-cost chemical sensors, which are ideal for continuous monitoring of disease status, to optical spectroscopy and state-of-the-art, high-resolution mass spectrometry. The latter can be utilized for untargeted analysis of exhaled breath, with the capability to identify hitherto unknown molecules. The interpretation of the resulting big data sets is complex and often constrained due to a limited number of participants. Even larger data sets will be needed for assessing reproducibility and for validation of biomarker candidates. In addition, molecular structures and quantification of compounds are generally not easily available from on-line measurements and require complementary measurements, for example, a separation method coupled to mass spectrometry. Furthermore, lack of standardization still hampers using the technique for screening larger cohorts of patients. The present review summarizes the present status and continuous improvements of the main on-line breath analysis methods, and evaluates obstacles for its wider application.
\end{abstract}

\section{INTRODUCTION \\ 1.1 A BRIEF HISTORICAL PERSPECTIVE .................................... 2 \\ 1.2 VOLATILE ORGANIC COMPOUNDS AND THE RESPIRATORY

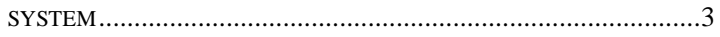 \\ 1.3 REPORTED VOLATILE ORGANIC COMPOUNDS BY ON-LINE \\ BREATH ANALYSIS ........................................................ 3 \\ 1.4 WORKFLOW FROM BREATH SAMPLING TO MOLECULAR \\ MARKERS.}

2. ANALYTICAL METHODS

2.1 SIFT-MS

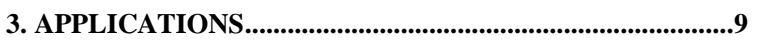

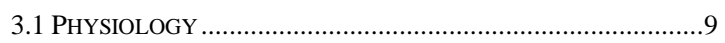

3.2 RESPIRATORY DISEASES .............................................. 9

3.2.1 Asthma ............................................................. 9

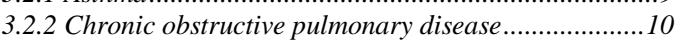

3.2.3 Cystic fibrosis ...................................................... 10

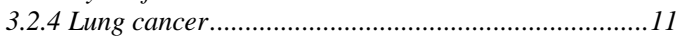

3.2.5 Pneumonia..............................................................11

3.2.6 Sleep apnea and related diseases..............................12

3.3 DRUG PHARMACOKINETICS ........................................ 12

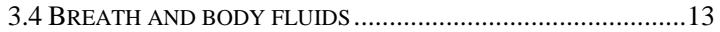

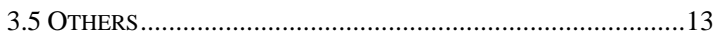

4 PERSPECTIVES
4.1 PROMISE AND IMPACT

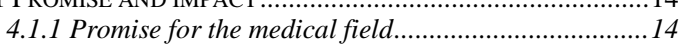

4.1.2 Promise for infants and children ............................. 14

4.1.3 Promise for sports and doping control .................... 15

4.1.4 Promise for recreational drugs............................... 15

4.2 CHALLENGES AND PITFALLS ............................................ 15

4.2.1 Deploying on-line breath analysis in a clinical

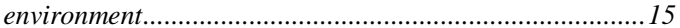

4.2.2 Contaminations and confounders ............................. 15

4.2.3 The issue of reproducibility and the importance of

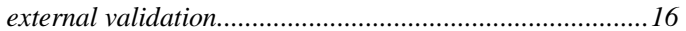

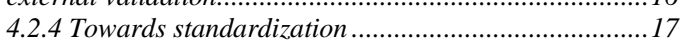

4.2.5 Quantification............................................................ 17

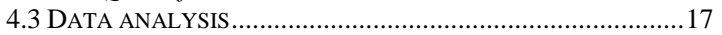

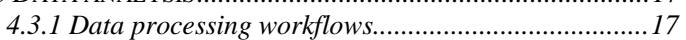

4.3.2 Statistical considerations............................................. 18

4.4 COMPOUND IDENTIFICATION AND METABOLIC PATHWAYS

.

4.5 MINIATURIZATION, PORTABILITY AND COSTS REDUCTION 20

5 CONCLUSIONS AND OUTLOOK.....................................20

6. ACKNOWLEDGMENTS.....................................................20

7. SUPPORTING INFORMATION AVAILABLE ................21

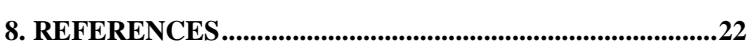

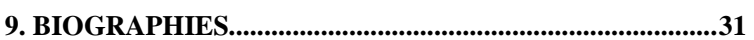




\section{Introduction}

On-line analysis of exhaled breath, sometimes called "real-time" or "direct" breath analysis, has gained a lot of momentum in recent years, and could in the future become the method of choice for some applications in medical diagnosis and personalized medicine. Getting insight into a person's metabolic status via on-line analysis of exhaled breath is significant and advantageous, because a wide range of compounds can be detected, because sampling is easy and completely non-invasive, and, as opposed to sending samples to a lab, the results are available on the spot. Breath is available in nearly unlimited quantities, its analysis presents no burden to the subject being measured, and its on-line analysis allows continuous monitoring of metabolic health, disease progression, and medication in short time intervals. Moreover, this approach might become indispensable in certain critical situations, e.g., in the emergency room, for correct treatment of a dangerous infection such as pneumonia, when consumption of potentially dangerous party drugs must be ascertained, in doping control, or in other situations where immediate action is necessary.

On-line analysis of exhaled breath could potentially reveal novel biomarkers for diseases, allow disease phenotyping, might become an inexpensive replacement for established tests, permit bedside or even home monitoring, and allow cost-effective and frequent checks of disease progression, effectiveness of a therapy, and adherence to medication. It could also open up completely new applications, e.g., diagnosis of asthma in young children or chronobiology where continuous sampling is required (e.g. sleep studies). There are, of course, challenges as well. Exhaled breath analysis if far from being established and standardized, and is currently only routinely used in clinical application for a select few applications (chapter 1.1). Biomarkers candidates that have been found in casecontrol studies await validation with statistically significant sample numbers, to avoid problems with overfitting due to limited sample sizes. The lack of standardization and reporting also extends to the level of data treatment, in particular for data processing and compound identification. There are several analytical methods that could be employed, but all of these still have issues with repeatability, batch effects, time drifts, and confounding factors. Requirements for low cost and portability of instrumentation are at odds with those for very high sensitivity, large dynamic range to detect metabolites with widely different concentrations, and chemical selectivity to analyze the complex mixture of compounds found in exhaled breath.

This review therefore focuses on sensitive, state-ofthe-art analytical methods that have the required chemical selectivity to differentiate molecules that occur in exhaled breath, and on their applications. We emphasize methods that allow to identify panels of molecules as opposed to established, singlecompound methods, e.g., an ethanol test (which will be mentioned in passing). The focus of the review is on on-line measurements, which automatically excludes off-line approaches that involve a twostep process of sample collection and storage, for example, using bags or Tenax tubes followed by sample analysis. In fact, due to complications with chemical stability (catalytic reactions on surfaces of collection devices; photochemical or thermal degradation; adsorption losses; etc.), on-line analysis is often the method of choice for analysis of exhaled breath. We use the term "on-line" breath analysis throughout the manuscript compared to "real-time" or "direct" breath analysis which are often used in the literature. The term "real-time" is not sufficiently defined without a given, debatable timescale. The term "direct" can be attributed to many things while "on-line" stands in clear contrast to "off-line" approaches. For certain applications only few on-line studies have been completed so far. Therefore, in these cases we included selected important off-line studies as a reference point (mostly GC-MS). These references have clearly been introduced as "off-line" studies throughout the manuscript. Some aspects of breath analysis have been recently covered in other reviews. Most notably, Wallace, and Pleil reviewed methods and instrumentation for analyzing exhaled gas-phase, condensate, and aerosols for use in clinical and environmental health applications. ${ }^{1}$ Direct, i.e., non-separative mass spectrometry methods for non-invasive diagnostics based on volatile organic compounds (VOCs) were reviewed by Casas-Ferreira et al. ${ }^{2}$, Giannoukos et al. summarized applications for security and biodiagnostics ${ }^{3}$ and Ahmed et al. covered the analysis of exhaled volatile organics that play a role in infection. ${ }^{4}$

\subsection{A brief historical perspective}

Breath analysis has been known since a long time. One of the first applications mentioned in writing was probably from Chinese medicine where diseases are characterized based on different smells. ${ }^{5}$ The famous physician Socrates mentioned characteristic smells for different types of imbalances/diseases such as a sweet smell (diabetes), a fishy smell (liver problems), a urine-like smell (kidney problems) or a putrid smell (lung abscess). ${ }^{6}$ One of the first instrumental approaches in a laboratory setting was developed by Lavoisier during the time of the French revolution when he described breath as a chemical reaction of respirable air $\left(\mathrm{O}_{2}\right)$ producing acid forming fixed air $\left(\mathrm{CO}_{2}\right){ }^{7}$ In 1971 Linus Pauling analyzed frozen breath with gas chromatography and could differentiate more than 250 volatile features. ${ }^{8}$ Today he is considered the father of modern breath analysis. Gas chromatography coupled to mass spectrometry in turn allowed much higher selectivity and compound identification. It is currently the most frequently used breath analysis method. Although it is limited because it is an off-line method, which can 


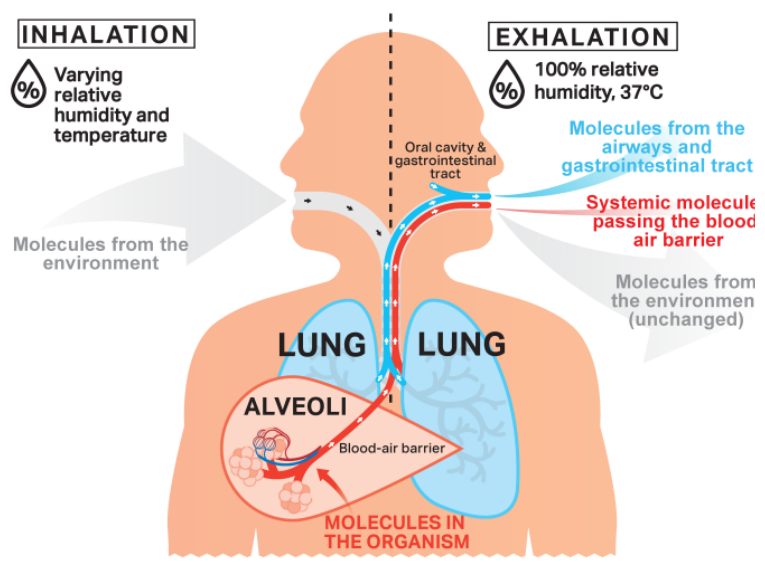

Figure 1: Pathway of exhaled molecules in the human body. A small proportion of exhaled molecules origins from the airways, gastrointestinal tract and the organism (i.e. systemic molecules passing the blood-air-barrier in the lungs). The majority of molecules in exhaled air is of environmental origin. Due to the maximal relative humidity and body temperature of $37^{\circ} \mathrm{C}$ the MSanalysis of exhaled breath may only be compared to a limited extend.

lead to sample loss and degradation during storage and transport. ${ }^{9}$

The three main mass spectrometry methods for online analysis of volatile organic compounds and later breath analysis are SIFT-MS and PTR-MS and more recently SESI-MS (chapter 2). The coupling with state-of-the-art high-resolution mass spectrometers opened up new possibilities with a much higher number of several thousand detected features and the capability for unknown identification. ${ }^{10}$

Portable breath analyzers are also considered important; this challenge has mainly been addressed by chemical sensors. However, sensors can currently only detect a very limited number of compounds in simple gas mixtures (chapter 4.5).

Only a limited number of breath analysis tests are currently used in patients and recognized by international guidelines. These include the ethanol breath test ${ }^{11}$, the nitric oxide breath test developed by Gustafsson et al. ${ }^{11}$ for the monitoring of asthma ${ }^{12}$, the hydrogen breath test to diagnose small intestinal bacterial overgrowth ${ }^{13}$ and the urea breath test for helicobacter pylori infection, ${ }^{14}$ At present, there is not a single exhaled breath test that is capable of diagnosing a disease as a stand-alone test.

\subsection{Volatile organic compounds and the respiratory system}

The human respiratory system emits a vast number of volatile organic compounds (VOCs) of different origin (see Figure 1). VOCs can either be endogenous, i.e., they arise from the respiratory tract or they are of systemic origin after passing the bloodair-barrier, or VOCs can be of exogenous origin, in which case they originate from the environment and are inhaled and exhaled without alteration. Whether VOCs from the gastrointestinal tract (e.g. limonene), which often origin from symbiotic bacteria, are considered exogeneous or endogenous are currently a matter of debate. The exogenous VOCs from the environment in exhaled breath outnumber the endogenous ones by far ${ }^{15}$, however, the small number of endogenous VOCs including the one from the gastrointestinal tract are of high interest in the field of medicine.

The pulmonary alveolus represents the smallest unit of the respiratory tract where molecules pass the blood-air barrier (35 to $200 \mathrm{~nm}$ thick) via diffusion. ${ }^{16}$ Estimations of the total area of alveoli range from $75-150 \mathrm{~m}^{2}$. Currently, on-line breath analysis is capable of detecting $>500$ VOCs in exhaled breath, which have different origins. ${ }^{15}$

The identification and quantification of airway and systemic biomarkers have been of particular interest recently, to gain insight into airway physiology and human metabolism in a non-invasive fashion ${ }^{17}$. Therefore, in most studies exclusively the end-tidal phase of an exhalation was analyzed, when the flow reaches a plateau with VOCs at high concentration and contaminants at their lowest concentration level. 9 While VOCs from the airways are constantly emitted, a wide range of factors contribute to the ability of a VOC to reach a phase equilibrium on both sides of the blood-air barrier: polarity, solubility in fat, Henry's partition constant and volatility, to list the important ones. It is therefore understandable that different classes of molecules in the blood (e.g. hydrophilic molecules) display a unique diffusion pattern when it comes to crossing the blood-air barrier. ${ }^{1819}$ Furthermore, the concentrations of exhaled VOCs must be diminished by an amount equal to their concentrations in the environment (i.e. ambient air). Under ideal conditions, the concentration of certain VOCs (e.g. acetone, acetonitrile or plasma free amino acids) is directly proportional to their respective concentration in blood or urine. ${ }^{202122}$ Smoking behavior, age, bodymass-index and biological sex can affect the concentration of certain exhaled breath components by a cumulative factor of up to $10 .^{23}{ }^{24}$ While there is a guideline from the American Thoracic Society/European Respiratory Society Task Force on methodological issues regarding exhaled breath condensate collection proposing to account for basic factors ${ }^{25}$, there are no recommendations or guidelines for exhaled breath analysis yet. However the standardisation focus group chaired by $\mathrm{J}$. Beauchamp and W. Miekisch of the International Association for Breath Research (IABR) is working on developing guidelines for this purpose.

\subsection{Reported volatile organic compounds by on-line breath analysis}

In 2014, de Lacy Costello et al. reviewed volatiles detected in exhaled breath. ${ }^{15}$ They reported 872 VOCs in breath, amongst them alkanes, alkenes, alkynes, benzyl and phenyl hydrocarbons, alcohols, ethers, aldehydes, acids, esters, ketones, nitrogen containing volatiles, sulfur-containing volatiles, and halogen-containing volatiles. However, only a small 
subgroup of them has also been monitored in on-line. Most of the reported compounds were detected offline, by GC-MS, and often sample pre-concentration steps like Tenax tubes or SPME were required. ${ }^{9}$ In this review, we focus on on-line monitoring of molecules in exhaled breath. We have compiled a table of the compounds and compound classes which have been reported in breath by on-line analysis of exhaled breath. The analytical methods, level of identification certainty, and literature references are given for each reported compound (Supplemental table 1). A wide range of compounds have been reported from 17 compound classes: small volatiles (e.g. NO), alcohols, aldehydes, alkanes/alkenes/alkynes, amino acids, benzenoids, benzothiazoles, carboxylic acids, esters, fatty acids, furans, indoles, ketones, sulphur containing compounds, terpenes, inorganic compounds and "others".

Up to now, the majority of the compound classes can only be detected by mass spectrometric methods (13 out of 17 compound classes); much fewer are accessible using the optical methods and chemical sensors discussed in this review. Until 2014, on-line measurements were mainly carried out by PTR-MS and SIFT-MS. Since then, the list of identified compounds got longer. Here, SESI coupled to high resolution mass spectrometry contributed. ${ }^{26-29}$ One has to keep in mind that volatiles detected in exhaled breath are not exclusively endogenous, there are also quite some exogenous compounds such as acetonitrile $^{30}$ and trimethyl-silyl acetonitrile ${ }^{31}$ in smoker's breath or methyl- tertiary butyl ether. ${ }^{32}$

\subsection{Workflow from breath sampling to molecular markers}

A typical workflow for on-line breath analysis in a clinical setting is shown in Figure 2. The workflow is conceptually related to the previously published workflow from Boots et al. ${ }^{33}$ However it includes now two additional steps, the selection of the appropriate analytical platform and the final goal to develop a quantitative assay for a clinical environment and has been re-designed. The starting point is a well- defined research question or set of questions addressing an unmet diagnostic need. The selection of an appropriate instrumental platform amongst the different options for on-line breath analysis is important. The main differences are compound coverage, sensitivity, selectivity, robustness, portability and costs (chapter 2.7).

In most cases the first step in a clinical context will be an exploratory study (e.g. a cross-sectional or longitudinal study) with an appropriate study design. During the phase of data acquisition continuous quality control is essential. Data processing converts, filters and normalizes the data, resulting in a breath feature matrix e.g. molecular weight and intensity per subject (chapter 4.3). This matrix is the basis for further statistical analysis, which allow to extract biomarker candidates (chapter 4.3). The molecular identity of the structure of the resulting features need to be carefully elucidated until unequivocal compound identification is achieved (chapter 4.4). Once the molecules are identified and depending on the research questions at hand, the next step is often a metabolomics analysis of the identified panel of molecules. An exploratory study, which has been carefully evaluated in such a manner resulting in a panel of putative biomarkers should be followed by a validation study to generate clinically meaningful biomarkers. If this step is passed successfully as well, a quantitative assay (chapter 4.2) can be developed for implementation into daily clinical practice.

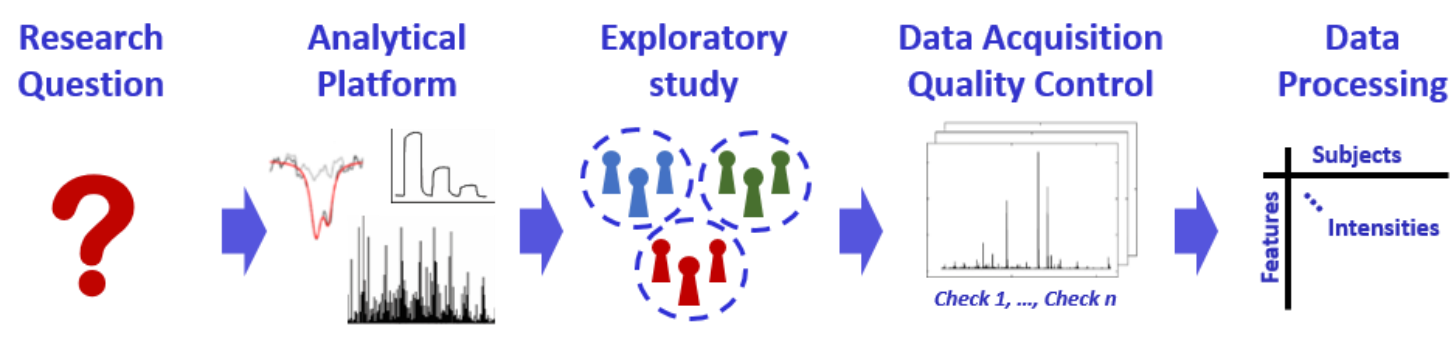

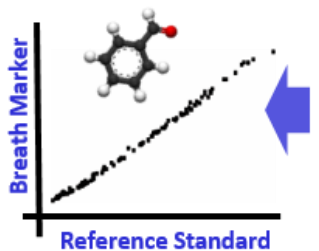
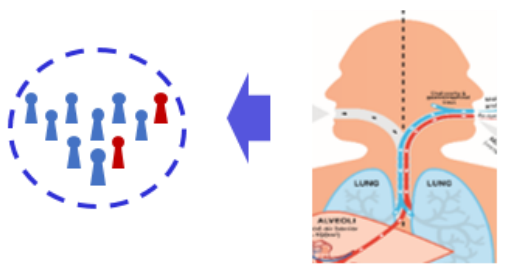

\section{Quantitative}

Assay

\begin{abstract}
Validation
\end{abstract} Study
Metabolomics Analysis
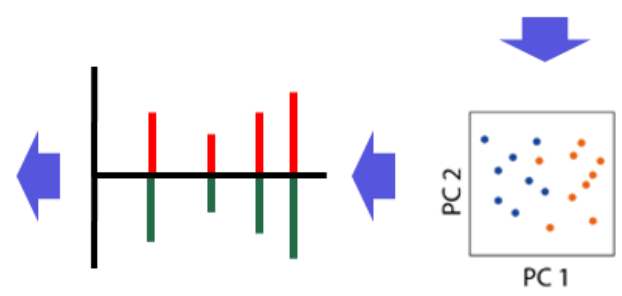

Figure 2: Workflow from breath sampling to molecular markers.

$$
\begin{aligned}
& \text { Compound } \\
& \text { Identification }
\end{aligned}
$$

Statistical

Analysis 


\section{Analytical methods}

\subsection{SIFT-MS}

SIFT-MS is a well-established method for breath analysis and there are several review and perspective articles available describing the technique and its applications in trace gas analysis. ${ }^{34-40}$ It has been applied extensively for studying the exhaled breath of healthy individuals ${ }^{21,41-61}$, but also in a number of clinical studies. ${ }^{52,61-69}$ Most studies make use of the strength of SIFT-MS for targeted analysis such as quantification of acetic acid in patients with gastroesophageal reflux disease ${ }^{68}$ or quantification of acetone in breath of type 2 diabetes patients. ${ }^{70}$

The principle of SIFT-MS is shown schematically in Figure 3A. It is based on chemical ionization. Briefly, reagent ions $\left(\mathrm{H}_{3} \mathrm{O}^{+}, \mathrm{NO}^{+}\right.$and $\left.\mathrm{O}_{2}^{+}\right)$are generated in a microwave plasma and the desired species is selected subsequently in a quadrupole filter. The analyte is then introduced into a heliumbuffered drift-tube, where it reacts with the reagent ions. The generated ions are detected by a quadrupole mass spectrometer. ${ }^{37,71}$

The ion chemistry in the drift tube is quite well understood and for many analytes kinetic constants are available. This knowledge allows for the direct translation of intensities into absolute analyte concentrations. However, for the analysis of exhaled breath, humidity has to be taken account. ${ }^{72}$ Hydrated reagent and analyte ions can occur as well as other adducts, making calculations more difficult. LOQs reported for SIFT-MS are in the range of low ppbv to high pptv ${ }^{73}$, the $m / z$ range up to $m / z=300^{37}$ and mass resolution is limited by the quadrupole mass analyzer. Hence, isomeric and isobaric compounds cannot be separated and untargeted metabolomics is difficult if not impossible. The only strategy of handling isobaric compounds is the use of several reagent ions in order to achieve selective ionization. ${ }^{74}$ Aldehydes and ketones, for example, can be separated due to the different chemistry of $\mathrm{H}_{3} \mathrm{O}^{+}$and $\mathrm{NO}^{+}$. ${ }^{75}$

In conclusion, SIFT-MS is mostly suited for targeted analysis. There, ion chemistry can be studied and accurate concentrations can be measured reliably. The most limiting factor remains the low mass resolution. However, recent developments include the implementation of time of flight mass analyzers $(\text { SIFT-TOF-MS })^{36}$ and the use of electric fields in the drift tube (SIFDT-MS) ${ }^{76}$ in order to combine the selectivity of SIFT and sensitivity of PTR .

\subsection{PTR-MS}

Originally, PTR-MS had been established in the field of environmental research and was mainly used with off-line sampling bags. ${ }^{77-79}$ However, the advantages of this fast method have also been recognized for on-
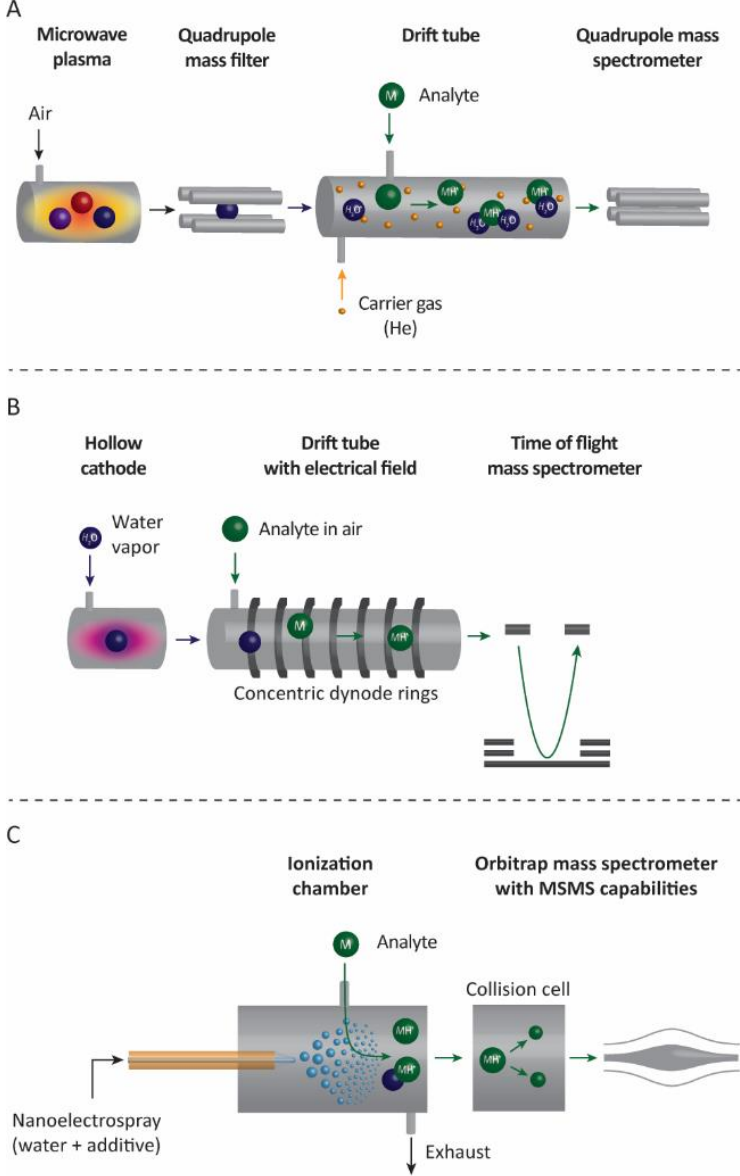

Figure 3: Schematic representation of different techniques used for breath analysis. a) SIFT-MS: In a microwave plasma reagent ions are generated from air and the desired species is selected subsequently in quadrupole mass filter. In a helium buffered drift tube, the analyte is ionized by reaction with the reagent ions. To date, ions are detected most commonly with a quadrupole mass spectrometer. b) PTR-MS: In a hollow cathode $\mathrm{H}_{3} \mathrm{O}^{+}$ions are generated from water vapor as reactive species. They are transferred into a drift tube with an electric field, where the analyte is introduced. PTR is mostly coupled to TOF-MS. c) SESI-MS: The analyte is introduced into a nanoelectrospray consisting of water and an additive. SESI can be coupled to high resolution mass spectrometers such as Orbitrap-MS or QTOF-MS.

line analysis of exhaled breath, ${ }^{80} 8182$ such that is increasingly used in this field.

Like SIFT-MS, PTR-MS relies on chemical ionization. Based on proton transfer from a reagent ion, the analyte sample is ionized and analyzed by mass spectrometry. However, in contrast to SIFTMS, mainly $\mathrm{H}_{3} \mathrm{O}^{+}$is used as proton source.

Early reviews have already described PTR-MS and its variations in detail, and have extensively covered the wide range of PTR-MS applications. ${ }^{83-85}$ As shown in a simplified schematic in Figure $3 \mathrm{~B}$, a plume of mostly $\mathrm{H}_{3} \mathrm{O}^{+}$ions is generated by $\mathrm{H}_{2} \mathrm{O}$ in a hollow cathode discharge. In a following source drift tube, the ion plume is further purified by reactive collisions with $\mathrm{H}_{2} \mathrm{O}$. Without an additional quadrupole mass filter as applied in SIFT-MS (see Figure $3 \mathrm{~A}$ ), the resulting pure reagent ions plume is directly introduced into a drift tube where the ionization of the gaseous analyte sample takes place. Subsequently, sample ions are analyzed by a mass 
spectrometer. Due to the fixed drift tube length, the reaction time for proton transfer from the reagent ions to the sample is fixed as well and hence allows for quantitative analysis. Within the drift tube, however, fragmentation of VOCs as for terpenes is known to occur that substantially complicates spectral analysis as well as absolute quantitation. ${ }^{86}$ Compared to SIFT-MS, superior sensitivity is obtained with PTR-MS since the sample air itself is acting as buffer gas in the drift tube and hence evades sample dilution.

The limit in detectability is set by the principle of PTR-MS itself. Only VOCs that feature a higher proton affinity (PA) than that of $\mathrm{H}_{2} \mathrm{O}$ can be ionized by proton transfer and hence be detected with this method. Recent research is bypassing this limitation by introducing further reagent ions into the hollow cathode such as $\mathrm{NO}^{+}$and $\mathrm{O}_{2}{ }^{+} \cdot{ }^{87}$ Thereby, the range of detectable VOCs is not only enlarged by compounds with lower PA than $\mathrm{H}_{2} \mathrm{O}$ but also isomeric compounds can be separated similarly to SIFT-MS. While ion pre-selection is not required for traditional PTR-MS using $\mathrm{H}_{3} \mathrm{O}^{+}$reagent ions, quadrupole mass filter became valuable again with this extension of reagent species allowing for fast switching between the species. This instrumental combination is realized in the selective, initially switchable, reagent ionization MS (SRI-MS). ${ }^{87}$ More recently, also a setup using $\mathrm{Kr}^{+}$or $\mathrm{Xe}^{+}$reagent ions has been described. $^{88}$

Mass accuracy of PTR-MS has greatly been improved by technical progress such as the introduction of TOF mass analyzers (PTR-TOF MS) and even more recently the hyphenation of a quadrupole to a TOF (PTR-QiTOF MS). ${ }^{79},{ }^{89},{ }^{90} \mathrm{New}$ PTR-TOF instruments are capable of mass resolving power higher than $\mathrm{m} / \Delta \mathrm{m} \quad 10,000{ }^{91}$ which is fundamental for compound identification as described in a subsequent chapter.

\subsection{SESI-MS}

In 1986 and 1994 the group of Fenn and the group of Hill discovered that electrosporay ionization (ESI) not only ionizes molecules dissolved in the spray solvent, but also compounds present in the environment of the ionization region. ${ }^{92} 9394$ They realized that the primary ions formed by ESI can induce a secondary ionization of gas-phase molecules that are brought into contact with the electrospray plume. This was eventually described as a separate technique by Hill and co-workers in 2000 and dubbed Secondary Electrospray Ionization-Mass Spectrometry (SESI-MS). ${ }^{95}$

A schematic drawing of SESI-MS is depicted in figure $3 \mathrm{c}$. A clean solvent containing an additive to increase conductivity is electrosprayed to generate primary ions. The analyte vapors are then guided to the electrospray plume, leading to ionization via proton-transfer reactions. This process is very soft, yielding predominantly the molecular ion for most chemical classes. ${ }^{96,97}$ However, the mechanism is not fully understood to date. While it was initially believed that gas-droplet interactions play a key role in the process, most data nowadays suggest ionmolecule reactions in the gas-phase, with charged water clusters as the primary ionizing species. ${ }^{398}$ Several factors can influence the ionization process, including sample humidity and the type of ESI solvent being used ${ }^{96,99-103}$. SESI-MS has been found to be most sensitive for polar compounds, with sensitivities reaching sub-ppqv values. ${ }^{104}$ Besides the outstanding efficiency and softness of the ionization process, the biggest strength of SESI is its modularity. Since it operates at ambient pressure it can be coupled to any pre-existing ambient inlet mass spectrometer, which allows for the usage of state-of-the-art high-resolution mass spectrometers with MS/MS capabilities. This resulted in the highest mass resolutions $(>140,000)$ and mass ranges $(\mathrm{m} / \mathrm{z}>$ 600 ) being reported for on-line breath analysis to date. ${ }^{31}$ Furthermore, the similarity to ESI makes it possible to perform unambiguous compound identification by using high-performance liquid chromatography-MS (HPLC-MS) measurements of exhaled breath condensate (EBC) as a complementary technique. ${ }^{29}$ The main drawback of SESI is that absolute quantification in the gas phase is not yet possible.

Despite SESI being a rather recent technique, a variety of applications have been published, including the detection of illicit drugs ${ }^{95}$, warfare agents ${ }^{105}$, and explosives ${ }^{102,106}$, as well as the analysis of food and skin ${ }^{100,107}$, bacterial cultures ${ }^{108}$, and most prominently exhaled breath. ${ }^{29,99,109-113}$

\subsection{Optical Methods}

There are several excellent reviews on optical spectroscopic methods for breath analysis, some of them quite recent ${ }^{114-117}$. Predominantly, absorption spectroscopy in the mid or near infrared is employed, while there are hardly any applications of Raman spectroscopy and UV/VIS spectroscopy related to exhaled breath analysis ${ }^{118,119}$. An established method that relies on optical detection, although chemiluminescence rather than spectroscopy, is the measurement of fractional exhaled nitric oxide (FeNO), a marker for bronchial inflammation that is determined in clinical practice when dealing with asthma. ${ }^{120}$

Normal, single pass absorption spectroscopy does not have sufficient sensitivity, and non-dispersive infrared (NDIR) spectroscopy can only be applied in special situations. However, narrow bandwidth IR / NIR laser spectroscopy (tunable diode lasers = TDLAS, quantum cascade lasers, optical frequency combs) perform quite well, due to their excellent spectral resolution characteristics. To increase sensitivity, multi-pass absorption cells are often employed, for example using cavity ring-down spectroscopy (CRDS), which increases the effective optical path length by 10,000 times or more. There are variants of CRDS, integrated cavity output 
spectroscopy (ICOS), cavity leak-out spectroscopy (CALOS), cavity-enhanced absorption spectroscopy (CEAS), off-axis cavity-enhanced absorption spectroscopy (OA-CEAS), which are essentially identical but are named differently in the literature. For example, NO, a well-established biomarker, was measured with a CALOS method using a $\mathrm{CO}$ sideband laser near $5 \mu \mathrm{m}$ wavelength and an optical cavity with two high-reflectivity mirrors $(\mathrm{R}=$ 99.98\%). This allowed simultaneous on-line detection of ${ }^{15} \mathrm{NO}$ and ${ }^{14} \mathrm{NO}$ in nasal air, with a record LOD of $7 \mathrm{ppt}$, using a 70-s integration time 121. An alternative is photoacoustic spectroscopy (PAS), a background-free method where a modulated laser generates sound waves in an absorption cell containing the gaseous (breath) sample, which are then detected with a microphone. LODs lie between $1 \mathrm{ppm}$ and $1 \mathrm{ppb}$, depending on laser power and cell design.

Generally, only small molecules (2-5 atoms) with well-defined, narrow absorption lines can be measured by optical methods, for example $\mathrm{CO}, \mathrm{NO}$, $\mathrm{NO}_{2}, \mathrm{H}_{2} \mathrm{O}, \mathrm{HCN}, \mathrm{NH}_{3}, \mathrm{CH}_{2} \mathrm{O}, \mathrm{CH}_{4}$, etc. Some characteristic absorption bands are shown in Figure 4 (middle panel). Larger molecules (> 6 atoms) have broader, more complicated absorption features, which often cannot be covered by scanning a single narrowband laser and/or show spectral congestion. The largest molecule detected by optical spectroscopy, specifically CRDS, was acetone, $(\mathrm{m} / \mathrm{z}$ $=58$ ) with a limit of detection (LOD) of $57 \mathrm{ppb}^{124}$, close to that of sensor systems, with LODs in the low ppb range ${ }^{125}$. These LODs are sufficient for practical applications, because acetone concentrations in breath are usually between $0.2-1.4 \mathrm{ppm}$. A general problem associated with optical spectroscopy is the strong absorption by water vapor in exhaled breath and by other small molecules that are present in air (Figure 4, top panel).

Ideally, spectroscopic measurements of trace compounds in air are thus made in the "infrared atmospheric windows" between 8 and $14 \mu \mathrm{m}$, and in the visible to mid-infrared region between 0.2 and $5.5 \mu \mathrm{m}$. Narrow bandwidth TDLAS can sometimes be used even outside of the IR atmospheric window (Figure 4, bottom panel). Another shortcoming of optical spectroscopy is compound identification. For example, in a study on laser-based spectroscopy, 24 different compounds were listed that distinguished children with asthma from healthy controls, and 12 different compounds for differentiating CF ${ }^{126}$. However, this "identification" was merely based on finding molecules in a database on IR spectroscopy that had significant absorption in wavelength areas of the vibrational spectra that differed between asthma patients and controls, the groups and had previously been reported to occur in human breath.

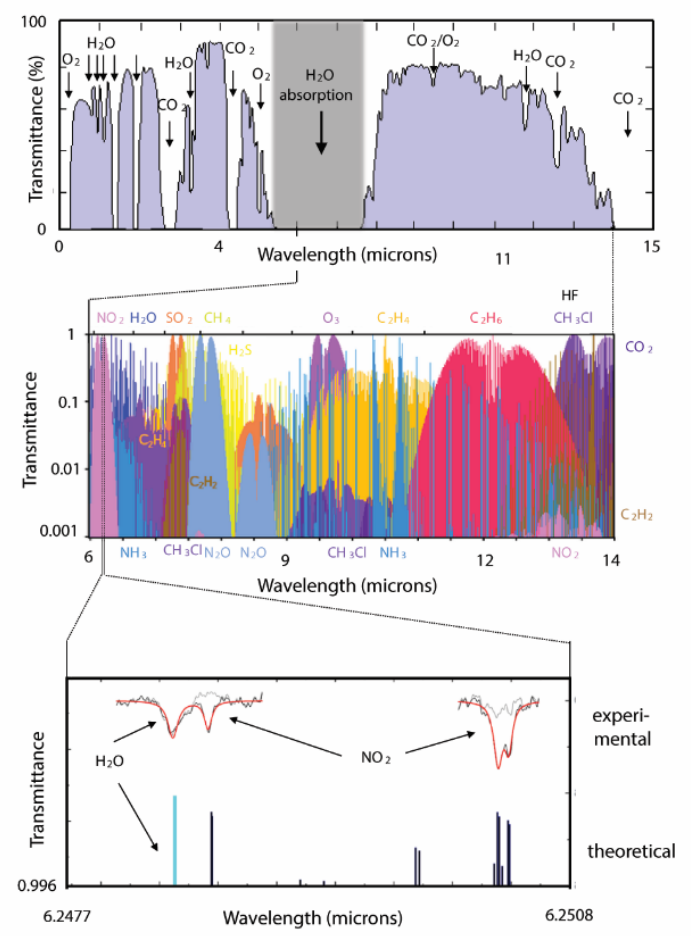

Figure 4: (Top panel) Transmission of the atmosphere. A significant fraction is obscured by strong absorption of water vapor (gray shaded area). Other compounds responsible for strong absorptions are marked also indicated (Adapted with permission from reference ${ }^{122}$ under CC PD 1.0 license). (Middle panel) Infrared absorption of common constituents and trace gases occurring in the atmosphere. Compounds and their spectral lines are color coded. From nanoplus.com. (Bottom panel) High-resolution quantum cascade laser infrared absorption measurement of $49 \mathrm{ppb}$ of $\mathrm{NO}_{2}$ in ambient air (red line) and $\mathrm{NO}_{2}$ scrubbed ambient air (grey line). The dark blue and light blue bars represent the theoretical line strengths of $\mathrm{NO}_{2}$ and $\mathrm{H}_{2} \mathrm{O}$ bands, which lie in a tiny fraction, $6.2477-6.2508 \mu \mathrm{m}$, of the IR spectrum. Despite being in the range of strong water absorption, the very high spectral resolution allows identification of NO (Adapted with permission from Reference ${ }^{123}$ under CC BY 3.0 license).

\subsection{Chemical sensors}

Chemical sensors hold the promise of small, portable devices at low costs for breath analysis. ${ }^{127}$ The strengths of chemical sensors are mainly their compact nature and low production costs per unit. There a three principal types of sensors: metal-oxide sensors $^{128}$, polymer-based sensors ${ }^{129}{ }^{130}$, and electrochemical sensors ${ }^{131}$. The use of carbon nanotube for chemical sensors has recently be reviewed. ${ }^{132}$. Figure 5 shows a size comparison from the nano-materials, to a single sensor element, up to the whole sampling device.

The most used chemical sensors for clinical applications are hydrogen sensors ${ }^{133}$ together with methane sensors ${ }^{134}$ to detect bacterial overgrowth and fructose malabsorption and nitric oxide sensors 135136 to detect fractional nitric oxide (FeNO) for asthma. ${ }^{137}$ Acetone sensors ${ }^{138,139}$ are used in the context of diabetes diagnosis. ${ }^{140}$ However, the claim of some acetone sensor developers of acetone sensors as a single biomarker assay for early diabetes diagnosis is pure speculation. ${ }^{141}$ The relationship between diabetes, type I or type II and acetone is 
more complex and additional markers will be needed to taken into consideration to develop a reliable diabetes breath assay. ${ }^{141}$ Hydrogen sulfide sensors are routinely used to assess halitosis (notably in Japan). 142,143 Sensors for carbon dioxide are established as a marker to determine end-tidal breathing. ${ }^{144}$

Current chemical sensors developments focus on ammonia $^{129,140}$ isoprene ${ }^{138,139}$, ammonia, formaldehyde $^{145}$ and even aldehydes ${ }^{146}$. Most of these sensors can detect concentrations in the lower $\mathrm{ppb}$ range. However only few of them have been tested with real breath samples. The detected compounds were mostly reported in well defined synthetic gas mixtures, e.g. mixtures of acetone, isoprene, ammonia and carbon dioxide often as a dry gas and not at breath relevant humidity levels which often affect sensor regeneration and stability. ${ }^{2,146}$, formaldehyde and even aldehydes. Most of these sensors can detect concentrations in the lower ppb range. However only few of them have been tested with real breath samples. The detected compounds were mostly reported in well-defined synthetic gas mixtures, e.g. mixtures of acetone, isoprene, ammonia and carbon dioxide often as a dry gas and not at breath relevant humidity levels which often affect sensor regeneration and stability. ${ }^{2,146}$, formaldehyde and even aldehydes. Most of these sensors can detect concentrations in the lower ppb range. However only few of them have been tested with real breath samples. The detected compounds were mostly reported in well defined synthetic gas mixtures, e.g. mixtures of acetone, isoprene, ammonia and carbon dioxide often as a dry gas and not at breath relevant humidity levels which often affect sensor regeneration and stability. ${ }^{2,146}$, formaldehyde and even aldehydes. Most of these sensors can detect concentrations in the lower ppb range. However only few of them have been tested with real breath samples. The detected compounds were mostly reported in well-defined synthetic gas mixtures, e.g. mixtures of acetone, isoprene, ammonia and carbon dioxide often as a dry gas and not at breath relevant humidity levels which often affect sensor regeneration and stability.

The main technical challenges are sufficient selectivity in complex breath mixtures, sensor regeneration and long-term stability. ${ }^{138,140,145}$ An important step for increased selectivity was the use of additional filter layers such as zeolite membranes 147 or activated alumina filters ${ }^{148}$ and the use of sensor arrays ${ }^{125}$. Furthermore, true sensor portability will only be achieved if the whole setup is miniaturized. Although individual sensors are compact in size, they can only work in combination with e.g. a heated sampling line for breath analysis. The development of a compact power supply for heating will pose a challenge.

It is also important to be aware of physiological constraints, e.g. for the ammonia sensor, that ammonia in breath is mainly produced orally from bacteria by urea and a such not in equilibrium with blood plasma which has been correctly stated by a recent sensor publication ${ }^{143}$ but often gets ignored in published works $^{129}$.
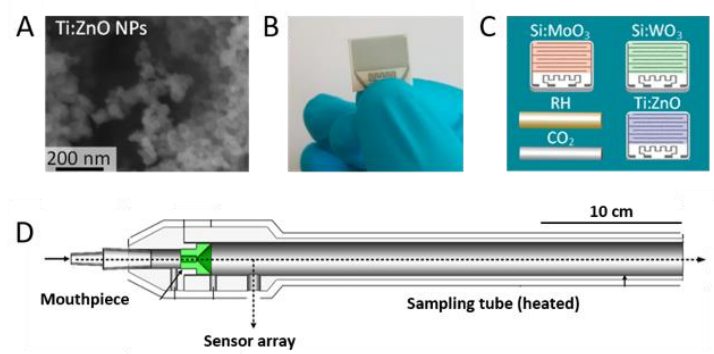

Figure 5: Schematic of a state-of-the art sensor array. A) Scanning electron microscopy picture of an example Ti-doped $\mathrm{ZnO}$ sensor, B) Photo of a single sensor, C) A sensor array example with three chemoresistive sensors for ammonia ( $\mathrm{Si}$ doped MoO3), acetone (Si-doped WO3), and isoprene (Ti-doped $\mathrm{ZnO}$ ) and a commercial humidity ( $\mathrm{RH}$ ) and $\mathrm{CO} 2$ sensor, D) Sampling setup with mouthpiece and heated sampling tube (power supply is not shown) (Figures A-C: Adapted with permission from reference ${ }^{129}$. Copyright 2018 American Chemical Society. Figure D: Adapated with permission from reference ${ }^{147}$. Copyright 2018 Elsevier).

\subsection{Synopsis of analytical methods and a comparison of their figures-of-merit}

Mass spectrometric methods are undoubtedly the most sensitive and information rich for on-line analysis of exhaled breath. For biomarker discovery and compound identification, high mass resolution is of key importance $(\mathrm{m} / \Delta \mathrm{m}>30,000$ is desirable). This performance is offered only by high-end, commercial mass analyzers, e.g. Orbitraps, which are normally large, expensive, lab-based instruments. They offer attractive options such as MS/MS, but require trained personnel for operation and are poorly suited for being deployed in a clinic or in the field. To take advantage of such an instrument, a modular, ambient pressure ionizer is required. Commercial options now exist, for example SESI (Super SESI, FIT, Spain; LF-SESI, SEADM, Spain) or plasma-based (SICRIT, Plasmion, Germany) ion sources. The classes of compounds that ionize well can differ depending on the ionization principle used, and quantitative measurements are not usually performed in campaigns where SESI or plasma ionization sources are utilized. Some of the analytical figures of merit (e.g. mass range) merely depend on the kind of mass spectrometer that is coupled to a particular ionization source. Traditionally, SIFT-MS and PTR-MS instruments have been operated with quadrupole mass analyzers that have limited mass range (typically $\leq 300$ ) and mass resolution (typically only unit mass resolution), although commercial PTR-MS instruments are now also available with higher mass resolution (Vocus PTR-TOF, TOFWERK, Switzerland; PTR-TOF 6000, Ionicon, Austria; see section 2.2). Especially PTR-MS instruments profit from rugged engineering, because of they are often designed for environmental applications that sometimes demand 
nearly unsupervised operation. They are therefore well suited for field applications. A common advantage of PTR-MS and SIFT-MS is also that quantitative results can be obtained, which is of great interest when comparing on-line MS measurements of exhaled metabolites with blood levels. Especially for SIFT-MS, quantitation based on known reaction kinetics is possible for selected compounds. In practice, calibration gas mixtures are required to obtain quantitative results from MS-based measurements, which is not trivial because of the very low concentrations of interesting metabolites in exhaled breath. The scan speed of mass spectrometers is typically about $1 \mathrm{scan} / \mathrm{sec}$., such that a very high time resolution is possible for breath analysis.

Instruments based on optical spectroscopy - offer more compact packaging and are less expensive than MS. However, the analytical performance is also compromised. LODs are in the low ppt range only for a few selected compounds ${ }^{124} 114$ normally ppb or even ppm detection limits are reached. The ability to identify compounds based on optical methods is also limited, as is the peak capacity, which is clearly lower than that of MS. The analysis of multicomponent mixtures or trace metabolites in a background of other compounds is difficult with optical spectroscopy. On the other hand, especially vibrational spectroscopy allows in principle the identification of small volatiles (supplemental table 1), even unknowns, because vibrational band structures are intimately linked to the molecular structure, and for small molecules can be fully understood and calculated. This also means that quantitation is in principle possibly based on first principles, although in practice, calibrants are required for quantitative measurements. The scan speed, dictated by the time required to average spectra, is typically in the range of a few seconds for optical spectroscopy, and sometimes even in the millisecond range $\mathrm{e}^{114}$, i.e. comparable to mass spectrometry.

Generally, the smaller and simpler the instrumentation, the lower the performance. The ultimate in simplicity and size are chemical sensors. However, the sensitivity of chemical sensors is only in the low ppb range ${ }^{145}$, some 3 orders of magnitude worse than that of mass spectrometry, and chemical identification is not possible. Also, there are issues with cross-sensitivity for other compounds than the ones a sensor is designed for. This problem can be partially alleviated by the use of filter layers, sensor arrays and smart, on-board algorithms that rapidly evaluate the response of such multi-sensor "chemical noses" or "electronic olfaction" 149-151. Typical response times of sensor-based detection systems are in the range of a few minutes, given by the recovery of the sensor surface. This time response is approx. two orders of magnitude slower than for MS and optical systems, but in many instances still fast enough for on-line monitoring of compounds in exhaled breath. The most attractive feature of chemical sensors is that they are are small, practical, and above all low cost. Thus, for selected compounds where the required LODs are not too low, sensors are presently the only option for mass production, for use as "wearables" or in combination with personal electronics such as smartphones.

\section{Applications}

\subsection{Physiology}

The understanding of physiologic breath composition is just as important as diagnostic applications for diseases. For example, modern on-line SESI-HRMS detectors can trace VOCs from the tricarboxylic acid cycle, which is one of the most important metabolic pathways for cellular respiration in aerobic organisms. ${ }^{152}$ With such systems, compounds like fumaric, succinic, malic, keto-glutaric, oxaloacetic, and aconitic acids can be conveniently monitored in the human exhaled breath.

But also, the understanding of individual variabilities contributes to the progress in this field. Important online population studies in healthy volunteers established distribution reference ranges of concentrations of specific breath metabolites. The first on-line population-based study defined the physiologic concentration of seven metabolites, which is an essential requirement for recognizing abnormally high levels that are associated with particular diseases. ${ }^{42}$ Later on-line population-based studies investigated the influence of age, sex, body mass index and dietary intake. ${ }^{48}$

However, the biggest advantage of on-line breath analysis comes into play when sudden changes occur in human physiology. On-line studies have identified changes in exhaled breath composition during exercise and are even capable to monitor changes during sleep. ${ }^{153} 154155$ All these studies contribute another layer to the understanding of human physiology and consequently the interpretation of abnormal patterns in exhaled breath.

\subsection{Respiratory diseases}

\subsubsection{Asthma}

Asthma is the most common chronic airway disease in children with a global estimated prevalence of asthma symptoms of $11.6 \%$ to $13.7 \% .^{156}$ Early diagnosis is associated with improved symptom control, function in everyday life and decrease of risk of exacerbations. ${ }^{157}$ International guidelines exist for asthma diagnosis, treatment and monitoring with lung function as standard for asthma diagnosis. ${ }^{158}$ However, lung function is largely impossible in children younger than four years old. Thus, diagnosis of asthma in this age group still is challenging depending mainly on patient history and assessment of risk factors.

The identification of disease-specific molecules in exhaled breath would greatly improve the 
management of asthma in young children and reduce long-term sequelae and health care cost. Additionally, such biomarkers might reveal more insight into the molecular pathophysiology of the different phenotypes of wheezing and other airway disorders.

So far, the only biomarker of breath that is currently used in clinical routine in Asthma is FeNO. It has been shown that NOS2 expression on airway epithelial cells is the most important contributor to FeNO. ${ }^{159}$ Increased levels of nitric oxide are linked to eosinophilic inflammation of the airways 12160 and therefore, associated with allergic asthma. FeNO is usually measured on-line using chemiluminescent analyser or electrochemical sensors. Several studies showed that increased FeNO concentration in preschool children is associated with persistence of wheeze in atopic children and predictive of the

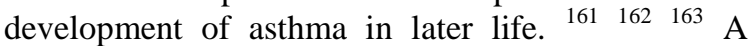
recent review including 175 studies evaluated the diagnostic value and the clinical utility of FeNO for the management of asthma in adults and children. The diagnostic value is influenced by atopy, asthmatherapy, age and smoking habits. Depending on the cutoffs used sensitivity ranges between 0.41 to 0.79 and specificities between 0.72 to 0.94 . Levels of FeNO are predictive for asthma worsening and acute exacerbations and show a weak association with asthma control. Randomized controlled studies showed that asthma therapy guided by FeNO resulted in fewer exacerbations but did not affect rates of asthma related quality of life or hospitalizations. ${ }^{137}$ The available literature was recently reviewed describing the results of 12 studies with off-line analysis, mostly using GC-MS. ${ }^{164}$ Compiling the data from the above-mentioned offline studies a total of 111 VOCs were so far reported to be altered in children with wheezing or asthma compared to controls. 165-167 However, these compounds were not found consistently in the different studies as only 13 of the VOCs were found in more than one study. ${ }^{164}$ This very limited overlap between studies even from the same research centers emphasizes the necessity for validation studies. The heterogeneous study populations (age ranges, phenotypes), the lack of standardization and different MS methodologies may explain the limited overlap to some extent.

Data from adult studies regarding Asthma is scarce with only three published off-line studies with either GC-MS or the pattern based electronic nose technology. ${ }^{168169170}$

\subsubsection{Chronic obstructive pulmonary disease}

Chronic obstructive pulmonary disease (COPD) is a chronic and progressive airway disease characterized by shortness of breath as well as cough, which is primarily caused by tobacco smoking. Long-term exposure to irritants causes chronic bronchitis, bronchiolitis, and irreversible emphysema in affected patients. In rare cases, nonsmokers who lack the alpha-1 antitrypsin protein can develop emphysema.
171. Therapy for COPD is symptomatic, as there is currently no cure for COPD. The highly prevalent lung-disease is the $4^{\text {th }}$ leading cause of death, and is expected to increase to the third leading cause of death in the world by 2030 .

The diagnostic "gold standard" for COPD is based on spirometry and subjective symptom assessments, similar to asthma with respective COPD guidelines. ${ }^{172}$ As it is primarily a disease of the lungs, a high number of breath-analysis studies are available in COPD, however only a minority of studies used online breath analysis techniques. ${ }^{173}$ Most studies use a case-control design with an untargeted approach in order to identify discriminative biomarkers for the disease or disease severity. Three on-line studies used MCC/IMS to identify COPD-specific biomarkers in a cohort of 119, 130 patients and 96 patients, respectively. ${ }^{174-176}$ While two studies reported a $70-79 \%$ accuracy in the diagnosis of COPD (based on 6-10 VOCs) the other study reported 10 biomarkers, most of which were unidentified. ${ }^{176}$ None of the identified substances overlapped between the two studies or could be validated in an external cohort.

Two studies reported a similar approach with the electronic nose technology. ${ }^{177,178}$ Finally, two on-line untargeted studies compared the breath print of COPD patients against healthy controls using SESIMS. Both studies yielded a receiver-operatingcharacteristic-curve with an area under the curve of 0.88 to 0.91 . However, in this case, with the aid of additional off-line methods some of the discriminate biomarkers could be identified and attributed to be metabolites of oxidative stress processes, such as fatty acids, aldehydes and amino acids. ${ }^{179,180}$

Most studies tried to control for the effects of smoking and there was evidence that lung capacity, body mass index, and certain inflammatory blood markers seem to play a role in the discrepancies observed between groups. ${ }^{174,181}$ In conclusion, studies on this topic are extremely heterogeneous and statistical methods for compound identification are not standardized. As a result, none of the reported biomarkers overlap between the studies and none of the biomarkers have been validated in an external cohort yet.

\subsubsection{Cystic fibrosis}

Cystic fibrosis $(\mathrm{CF})$ is the most common autosomal recessive disorder among Caucasians, caused by mutations (>2000 identified to date) in the CF transmembrane conductance regulator (CFTR) gene on chromosome $7 \mathrm{q} 31.2$ resulting in severely reduced mucus clearance. ${ }^{182}$ Cystic fibrosis diagnosis is confirmed by elevated sweat chloride and identified with newborn screening. ${ }^{183} 184$ Although normal at birth, the lungs of CF patients are rapidly colonized by pathogens including Staphylococcus aureus and/or Haemophilus influenzae followed by predomination of gram-negative bacilli such as Pseudomonas aeruginosa. ${ }^{185}$ Moreover, there is good evidence that in $\mathrm{CF}$ lungs there is an exaggerated 
inflammatory response to viral infections ${ }^{186}$, increased activity of neutrophils, and elevated levels of pro-inflammatory cytokines. ${ }^{187}$ The result is a vicious cycle of perpetuating infection, inflammation and airway destruction creating niches for bacterial growth and microbial treatment evasion alongside deteriorating lung function.

Early detection of airway infection is crucial not only to start antimicrobial therapy but also to reduce cross-infections. A recent systematic review summarized the known VOCs of infection. ${ }^{188}$ Another recent review summarized the VOCs released from bacteria and detected with mass spectrometric techniques. ${ }^{189}$

Despite the high potential of the analysis of exhaled volatile organic compounds and whole breath only a handful of studies applying different massspectrometry methods have been published so far. In all but one studies breath was collected off-line in tedlar or naphilone bags and then measured either by SIFT-MS or via up-concentration in sorption tubes, thermal desorption and analysis and with GC-MS.

Solid-phase microextraction, SPME and gas chromatography/mass spectrometric (GC/MS) analyses of VOC were used in two small pilot studies showing distinct patterns of a range of features in patients with CF. ${ }^{190,191}$ By using 22 VOCs out of 1099 VOCs assessed by GC-MS with a prevalence of at least $7 \%$ children with CF could be correctly distinguished from healthy controls with very good short time reproducibility over a $1 \mathrm{~h}$ interval. ${ }^{192}$ In addition, acetic acid vapour concentration measured by SIFT-MS was elevated in children with $\mathrm{CF}$ compared to healthy control. ${ }^{193}$ Due to different methodologies these off-line studies show nearly no agreement amongst each other regarding the detected molecules.

A first study with secondary electrospray ionizationhigh resolution mass spectrometry (SESI-HMS compared healthy controls and CF patients. A total of 49 compounds were found to be significantly different between the two groups and the two most discriminating compounds showed a specificity of $80.0 \%$ and a sensitivity of $63.3 \%$ with a prediction area under the receiver operating curve (AUROC) of $77.1 \%$ (95\% CI $62.2 \%-87.8 \%$ ). Significant features included markers of oxidative stress. ${ }^{194}$

\subsubsection{Lung cancer}

Lung cancer is the most common cause of cancerrelated death in men (second in women). The current "gold standard" tests to screen and diagnose lung cancer are computerized tomography (CT) scan. ${ }^{195}$ 196 While CT scans are a useful tool for detecting suspicious pulmonary nodules, they cannot be used alone to diagnose lung cancer. Rather, if a suspicious nodule is identified in a CT Scan, an invasive and costly biopsy must then be performed to determine whether the nodule is "malignant" (cancerous) or "benign" (non-cancerous). One central diagnostic dilemma in medicine is the lack of symptoms in the early stages of disease on the one hand, and the limited treatment options in the usually symptomatic end-stage on the other hand. Hence, there is clearly an unmet need for noninvasive screening test to detect asymptomatic patients at an early stage of disease.

Five untargeted on-line studies using PTR ${ }^{197-199}$ and $\mathrm{IMS}^{200,201}$ reported VOCs for diagnostic purposes in early stage lung cancer. One study reported lowered concentrations of isoprene acetone and methanol in patients with lung cancer. ${ }^{197}$ The biggest study (187 individuals) reported two unidentified peaks to be sufficiently discriminative for lung cancer. ${ }^{199}$ The two studies using IMS reported the VOC n-dodecane as a potentially useful biomarker or the combination of 23 different peak regions as $100 \%$ accurate in a cohort of 32 patients. ${ }^{200,201}$ Recently, one team using the Cyranose 320 (eNose) tried to identify patients with lung cancer in a cohort of 475 patients (sensitivity $95.8 \%$, specificity $92.3 \%$ in smokers) ${ }^{202}$. When results from off-line-methods are also taken into account, a total of 193 VOCs are reported in association with lung cancer, with a significant amount of VOCs overlapping between studies (e.g. nonanal etc.). ${ }^{203}$ On this basis, one group systematically investigated the 125 most reliable candidate-VOCs $^{204}$ out of a database ${ }^{15}$ as potential cancer-associated VOC markers. These VOCs were selected because they could be identified across studies and bodily fluids and they were also present in transformed human cells in vitro. ${ }^{204}$ The authors concluded that currently there is no single tumorspecific VOC for which clinical relevance could be proved. ${ }^{204}$ Currently only one clinical phase-II biomarker study for VOCs associated with lung cancer has been reported. ${ }^{205}$

\subsubsection{Pneumonia}

Community-acquired pneumonia is a common and potentially serious childhood infection and is still one of the most important causes of mortality in children. Almost 2 million children died from acute respiratory infections in the year 2000, most from pneumonia. ${ }^{206,207}$ Pneumonia is a clinical diagnosis, therefore no "gold standard" method applies. However, guidelines are available. ${ }^{208}$ Current diagnostic tests lack accuracy and are still discussed amongst clinicians.

A rapid, non-invasive test at bedside would be highly attractive. In particular to follow the strategy as proposed by the BreathDXConsortium ${ }^{209}$ : (1) to exclude pneumonia infection to avoid unnecessary treatment with antibiotics, (2) to identify present pathogen for specific treatment and (3) to monitor the response of the treatment. When investigating the detected volatiles, it is important to keep in mind their origin from either the microbial pathogen, a host immune response or from the host respective patient.

A recent systematic review summarized the current breath analysis approaches in the context of pneumonia ${ }^{209}$. Three GC-MS studies reported distinct volatile patterns related to pneumonia. ${ }^{210-212}$ 
However, they showed nearly no agreement amongst each other. Most metabolites were unique to one of the three studies and there was even disagreement with regard to change in concentration of heptane and ethanol. Here clearly an agreement upon the applied analytical workflow as well as subsequent validation studies are needed.

On-line measurements were so far only done with mice and SESI-MS analysis by Hill et al. ${ }^{213-216}$. Distinct panels of metabolites could distinguish infected from not infected mice. In addition, characteristic panels for a range of pathogens were reported. Furthermore, the origin of metabolites from either host or host response could be differentiated. ${ }^{216}$

The studies mentioned in the systematic review which investigated multiple compounds ${ }^{209}$ were all proof of concept studies and clearly validation studies are needed.

\subsubsection{Sleep apnea and related diseases}

Obstructive sleep apnea (OSA), a sleep-related breathing disorder, is caused by complete or partial obstruction of the upper airway, leading to obstructive apneas and hypopneas, which are associated with oxygen desaturations and arousals from sleep. This highly prevalent disease is an important public health issue, because patients suffer from excessive sleepiness, impaired quality of life, high blood pressure and an increased rate of accidents. The current "gold standard" for sleep apnea diagnosis are polysomnography and subjective symptom assessments with respective guidelines ${ }^{217}$. Currently, the diagnosis is established in a hospital sleep-study, which is expensive, time-consuming, and provides no information on the disease-specific metabolomics changes.

An on-line randomized-controlled-trial assessed the daytime metabolic consequences in patients with OSA. In an untargeted approach (SESI-MS), the application of on-line breath analysis allowed to compute a disease specific breath-print, by which OSA can be diagnosed within minutes and each individual's metabolic response to OSA can be tracked. ${ }^{111}$ Cross-sectional studies using an electronic nose describe the evening-morning patterns in patients with $\mathrm{OSA}^{218}$ and to which extent obesity might contribute to the breath-profile in patients with OSA. ${ }^{219}$ Three studies using a targeted approach (electronic nose) also assessed the performance of on-line breath analysis in children and adults. ${ }^{219-221}$ When attempting to diagnose OSA against the goldstandard (sleep laboratory test), AUROCs ranged from 0.84 (no $95 \%$ CI provided) ${ }^{220}, 0.85$ (95\% CI $0.75-0.96)^{221}$ and $0.87(95 \% \text { CI } 0.61-1.00)^{218}$, suggesting a potential role in future diagnostic applicability.

On-line breath analysis with PTR-MS has also proven to be applicable within the sleep laboratory environment. In an explorative study, researchers were able to track the exhaled breath for the whole sleep cycle and document metabolic changes, which corresponded to sleep events. ${ }^{153}$ For example, two biomarkers, acetone and isoprene, were highly specific for leg movements or the rapid eye movement sleep. ${ }^{153}$

In summary, on-line breath analysis by untargeted mass spectrometry and by chemical sensors have several important applications in the field and might one day facilitate diagnosis and therapy monitoring in patients. ${ }^{222}$ However, currently only few of the reported biomarkers overlap between the studies and statistical issues may have led to an overestimation of accuracy.

\subsection{Drug pharmacokinetics}

Besides diagnostic applications, a strong potential of on-line breath analysis has also been recognized for pharmacokinetics. To define optimal drug dosage and administration time, levels of administered drugs and its metabolized products in blood or plasma are commonly monitored over time. Despite wellestablished standard procedures, drug concentration in plasma can be strongly affected by variations such as in the Cytochrome P450 activity and/ or in the adsorption, distribution, metabolism and excretion (ADME) processes of the drug. Thereby, theoretically calculated drug concentrations might differ strongly from the actual concentration in blood. Further, commonly applied methods such as immunoassays or high-performance liquid chromatography (HPLC) for blood testing and exhaled breath condensate (EBC) for breath analysis are intense in sample preparation time and comprise off-line 223-226 $^{2}$

In this context, on-line breath analysis strongly increases the time resolution for drug monitoring. Instantaneously, metabolism and clearance of administered drugs can be followed non-invasively and at any given time point. ${ }^{227,228}$ This would allow to define the therapeutic window more precisely. Due to the low efforts, cost- as well as timewise, and based on the individual metabolic drug uptake that can be detected by on-line methods, individualized therapy with respect to personalized drug dosage and dosing time becomes even more realistic. ${ }^{227,228}$

Several studies have been performed on propofol using e.g. PTR-MS ${ }^{229}$ to evaluate the reliability of these on-line methods for pharmacokinetics in direct comparison with standard serum-based off-line methods. Similarly, feasibility studies on valproic acid using PTR-MS ${ }^{230}$ and SESI-MS ${ }^{231,232}$, eucalyptol using PTR-MS, ${ }^{233}$ salbutamol with SESI$\mathrm{MS}^{112}$ and more recently on ketamine and its metabolites using SESI-MS ${ }^{231,234}$ have been successfully performed. Moreover, on-line PTR-MS breath analysis in single mice and rats revealed that upon dietary intervention VOC levels were altered which are potentially linked to metabolic pathways. 235236237 170-172 Applying such on-line studies on mammalian model system for drug pharmacokinetic studies would greatly allow to reduce the number of animals used, thus also commonly observed inter- 
individual variations ${ }^{231,238}$ and to circumvent anaesthetics-induced bias (if no sedation is needed). ${ }^{237}$

While the before mentioned studies mirror quite well to what extend on-line breath analysis could improve and revolutionize pharmacokinetics, their implementation in this field of research is facing crucial issues. As outlined in detail by Beauchamp et al., the comparability of results from independent studies is not yet given for on-line breath analysis ${ }^{239}$. ${ }^{240}$. Besides standardizing breath sampling procedures, the classification of endogenous and exogenous breath compounds turned out to be nontrivial. Breath composition and compound concentrations can strongly be affected by external factors such as recent activities, dietary intake, medication as well as environmental exposure. These factors not only complicate indicating breath markers to a specific health state. They demonstrate additional necessary parameters for suitable matched control groups in clinical studies to avoid data misinterpretation.

\subsection{Breath and body fluids}

As already discussed, on-line breath analysis may be not just able to detect biomarkers of lung diseases (e.g. COPD, pneumonia, etc.) originating from the respiratory tract, but also identify VOCs that are systemic, i.e., reflecting concentrations in the blood stream. Therefore, the underlying mechanism for the application of on-line breath analysis in systemic and/or metabolic diseases is the assumption that a sound correlation between blood and breath levels of VOCs of interest exists. In some cases (e.g. ethanol), the analysis of exhaled breath has already proven to be equally reliable when compared to blood sampling. ${ }^{241}$ Other classes of biomarkers (e.g. long chain amino acids) have physical or chemical properties that simply does not allow them to pass the blood-air-barrier, or they are diluted in exhaled breath below the limit of detection. Reported studies of each bodily fluid report similarities between them, however every fluid seems to have some distinct properties and $<1 \%$ of VOCs are ubiquitous across all bodily fluids (incl. breath, saliva, blood, breast milk, skin, urine, faeces). ${ }^{15}$ Unfortunately, a major limitation in the field of breath-analysis is the currently unknown origin and metabolic context of VOCs of interest. ${ }^{204}$

In a direct comparison, off-line studies suggested that mainly hydrocarbons, ketones, terpenes, heterocyclic compounds and aromatic compounds are present in comparable concentrations (linear relationship) on both sides of the blood-air-barrier and are not just a byproduct of room-air. ${ }^{242}$ Additionally, there is robust evidence that VOCs like acetone $^{243}$, isoprene ${ }^{244-246}$ (originating from muscle cells $^{247}$ ) and pentane ${ }^{246}$ but also anesthetics like propofol $^{245}$ are equilibrated at the blood-air-barrier and their breath-concentration reflects sudden fluctuations in the blood compartment.
Only few on-line breath analysis studies assessed the correlation of VOCs in exhaled air and other bodily fluids. One exploratory study using SIFT-MS reported a moderate correlation $\left(\mathrm{r}^{2}=0.5\right)$ between blood, urine and exhaled breath for acetonitrile, a VOC which is distributed throughout the body fluids. ${ }^{21}$ Another exploratory study using SESI-MS reported a correlation of eight slightly volatile amino acids between exhaled breath and blood. Notably in this study the results could be confirmed in a prospective validation cohort study and the coefficients of variation were comparably low to the current gold-standard method in blood (highperformance liquid chromatography). ${ }^{27}$ The reported amino acids are of clinical interest, since they are routinely measured in hospital laboratories for metabolic disorders.

Current evidence suggests that although some VOCs are ubiquitous in the bodily fluids, there are some challenges to face when it comes to exhaled breath: first, only a fraction of VOCs reported in exhaled breath in the literature are also detectable in human blood (approx. 10:1), therefore their significance regarding the human metabolism is often limited. ${ }^{15}$ In the future, the use of cell lines and cultures of microorganisms will play an essential role in understanding the origin of VOCs. ${ }^{204}$ Second, breath concentrations of VOCs are diluted, by a factor of approximately 100-100'000 (depending on the VOC) ${ }^{245,248}$, which requires extremely sensitive instruments. Third, unlike blood, breath analysis is susceptible to minor changes in ventilation/perfusion ratios of the lung and requires a standardized setup, which is not yet implemented in all studies. ${ }^{24,245,248}$ Fourth, depending on chemical and physical properties of the VOCs (e.g. Henry constant, lipophilic behavior), the changes in the blood compartment might occur in exhaled breath with a significant delay. ${ }^{155}$ On the other hand, there is considerable evidence for a linear relationship between VOC breath/blood concentrations ${ }^{27,242,245}$ and there is evidence that for some extremely volatile VOCs (e.g. acetone or certain amino acids) on-line breath-analysis might provide a better and more reproducible signal when compared to blood. ${ }^{27,243}$

\subsection{Others}

On-line analysis of VOCs is also in demand for security applications, as has been illustrated in previous reviews. ${ }^{3,249}$ To name only one out of several ongoing studies, a recently started Europeanwide project, the TOXI-Triage project, funded by the European Union's Horizon 2020 research, focuses on secure risk management of chemical, biological, radiological and nuclear incidences. One study has evaluated the applicability of GC-IMS to study VOCs in breath for early detection of intoxication and exposure to toxic industrial chemicals. Although we did not include GC-IMS in this review, the method has potential based on its portability and 
robustness for field studies. However, the main challenges for this technology are compound coverage, selectivity and analysis time. With respect to these three aspects other MS based methods are superior. Therefore, it will need to be proven how to overcome these obstacles and detect intoxications at relevant trace levels for proper warning systems.

Another research field aims for detecting humanderived VOCs that create a human chemical signature and could serve as markers for the presence of human life. ${ }^{250}$ Such a VOC-based chemical signature for humans could be of tremendous help for scenting and rescuing humans after catastrophes such as earthquakes. For this purpose, potential VOCs for human biomarkers have already been summarized. ${ }^{251}$ In the context of fighting smuggling of humans, portable devices with high sensitivity and fast analysis time will become essential, since the most common procedure using search-and-rescue dogs is time-consuming. These projects illustrate well the variety of applications for breath analysis in the security sector.

\section{Perspectives}

\subsection{Promise and impact}

\subsubsection{Promise for the medical field}

Preliminary data from the field of on-line breath analysis suggest a broad range of applications beyond the task of predicting a diagnosis ${ }^{17}$ :

1) The ability of (theoretically) unlimited sequential sampling allows to closely monitor changes in the human metabolism down to a time resolution of $20-100 \mathrm{~ms}$ (e.g. to monitor sudden metabolic changes during exercise or the REMphase in sleep ${ }^{153,155}$ ).

2) Phenotyping of patients as a step towards individualized medicine, particularly for complex diseases (e.g. nitric oxide measurements in asthma patients $^{12}$.

3) The ability of breath analysis to track the circadian rhythm ${ }^{234,252}$ may play a major role in chronopharmacology - a field where treatment is delivered according to the circadian rhythm in order to maximize its effectiveness.

4) Drug identification and quantification in breath is a highly attractive tool to study pharmacokinetics, drug monitoring and therapeutic effects of biologicals. ${ }^{253}$ Besides the well-known breath alcohol test, this technology has potential to accurately track concentrations of administered drugs and monitor its pharmacokinetics. ${ }^{95,230,240,245}$.

5) A cornerstone in the management of chronic diseases is monitoring of disease activity. For example, sequential sampling via breath analysis may help patients with amino acid diseases (e.g. phenylketonuria) to get instantaneous feedback and adequately adjust their therapy (i.e. diet) ${ }^{27}$ or patients with diabetes to adjust their insulinregime.
6) Independent of other metabolomic sampling methods, on-line breath analysis has proven to advance our understanding of pathophysiology and disease-mechanisms (e.g. the tryptophanpathway). ${ }^{254}$

Currently, most of these domains constitute rather a proof-of-principle than a sound diagnostic application and we are far from fully exploiting the entire potential of on-line exhaled breath analysis. Once panels of promising biomarkers with sufficient selectivity have been identified and externally validated, the development of selective nanosensors as a point of care testing can be implemented in clinics. $^{255}$

\subsubsection{Promise for infants and children}

Many chronic diseases start early in life and progress over childhood potentially leading to irreversible pathologies. Often there is a window of opportunity for early treatment interventions aiming at the reduction of disease progression. In contrast to older children and adults, diseases in infants and young children may have very heterogeneous phenotypes and atypical symptoms. Unambiguous diagnosis may be very difficult or even impossible due to the difficulties in obtaining objective physiological measures. An example is the measurement of lung function, which is technically very complex and demanding in young children as they lack active cooperation. In particular, breath analysis is of interest for the following applications for infants and young children:

1) Disease detection when a diagnostic test is not available (e.g. asthma diagnosis which is currently not feasible below the ages 4-5).

2) Rapid phenotyping when clear diagnostic markers are lacking (e.g. for different types of infections).

3) Monitoring of disease progression for effective treatment (e.g. lower airway infections).

Many diseases in childhood not only present a heterogeneous clinical picture but also vary largely in severity over time. For example, viral lower airway infections are very frequent in young children $^{256}$ and typically lead to exacerbations of chronic airway diseases. Timely and proper disease monitoring is therefore a critical issue. However, with a lack of objective measures one has often to rely solely on reports by the respective caregivers.

The analysis of exhaled breath has clear advantages due to its non-invasive character, safety and speed, making it especially interesting and attractive in young children. However, the analysis of panels of breath molecules is still in its infancy and limited to discovery phase research. ${ }^{166,257-263}$

Beside the measurement of FeNO some work has been done in healthy children using sSIFT-MS. Several metabolites could be measured in a study of 200 healhy children. For some levels were lower than previously found in adults including aceton, ammonia, methanol and isoprene. Isoprene was 
found to increase with age in the paediatric population. ${ }^{52} 48$

\subsubsection{Promise for sports and doping control}

A simple, rapid, non-invasive test which allows repeated respective continuous measurements during exercise evaluation would be highly attractive. ${ }^{264}$ Currently very limited work has been done in this field with regard to breath analysis. Changes in isoprene and acetone ${ }^{265}$ as well as methane concentrations have been detected during exercise. Furthermore, a change in a panel of 44 VOCs has been reported ${ }^{266}$. Such studies need to be especially carefully with the development of appropriate sampling devices for stationary training (e.g. facemasks) or portables (e.g. body sensors).

On-line breath analysis is also attractive for doping control. In particular as on-site sampling device which less prone to cheating such as with urine sampling. Some dopant drugs have been reported in breath by off-line analysis such as fentanyl, propofol and tetrahydrocannabionol ${ }^{253}$. There has been some preliminary work for on-line identification with SESI-MS for cocaine and atenolol ${ }^{267}$ but these compounds have not yet been investigated in breath. Doping control with on-line breath analysis is promising but also challenging mainly with regard to obtain sufficient sensitivity and selectivity which might focus on panels of the more volatile drug induced metabolites.

\subsubsection{Promise for recreational drugs}

Breath analysis would allow direct on-site sampling and is as such attractive for identification and quantification of recreational drugs, e.g., as an alternative for blood and urine sampling to test for drugged driving. So far only the alcohol breath test is established $^{11}$. There have been several studies with focus on smoking. In particular the recent legalization of marihuana in Canada, several states of the US and Europe resulted in the development of mobile prototypes for tetrahydrocannabinol ( $\mathrm{THC}^{268-}$ $270{ }^{253}$ ). Most recreational drugs are larger and less volatile molecules and are as such often analyzed as EBCs. Several compounds could be detected by trapping on cartridges or in EBCs such as: amphetamine and metamphetamine ${ }^{271}$ or methadone. ${ }^{272}$ Their detection required a continuous 10 min sampling on a cartridge followed by solvent extraction and subsequent LC-MS/MS analysis. Morphine, codeine, cocaine, diazepam, oxazepam could be detected as EBCs with micro-particle sampling. ${ }^{273}$ Relatively recent on-line methods have shown the capability to detect semi-volatile compounds such as amino acids ${ }^{27}$ or larger chain fatty acids $^{29}$. They might be applicable as an alternative to EBC for the identification and quantification of recreational drugs.

\subsection{Challenges and pitfalls}

\subsubsection{Deploying on-line breath analysis in a clinical environment}

Because of its generality, we envision on-line breath analysis based on mass spectrometry as a potential first-tier diagnostic for a range of diseases in a hospital setting. This approach would allow triage of patients, followed by more specific analyses and, if deemded necessary, more invasive and costly methods. On-line methods are rapid and deliver near immediate results, which can be important in certain situations (e.g. emergency room situation or drug monitoring). However, they are not yet on par with off-line systems in clinical laboratories that operate high-throughput analyses, for example by running large batches of samples overnight.

One requirement for the current rather bulky mass spectrometers is a laboratory that is accessible for patients, preferably in a central location at a clinic or hospital. Furthermore, on-line breath analysis will require external validation studies to be accepted as a clinical quantitative assay. External validation will be more challenging than with off-line methods that allow samples collected at various sites and sample analysis in a central analytical laboratory. On-line studies will require the same instrumentation at each site with highly standardized procedures and trained operators. Although the efforts are considerable, such multi-center studies with on-line breath analysis methods have been launched at several research sites.

\subsubsection{Contaminations and confounders}

The speed of on-line analyses implies several drawbacks that need to be addressed. The lack of sample preparation leads to a highly complex matrix that can disturb the results via isobaric overlaps and matrix effects. ${ }^{274}$ Therefore, a high instrument specificity is required to obtain reliable signals. In sensor-based techniques, this is a major issue since developing appropriate materials is challenging. In mass spectrometric techniques, this can be addressed by using high-resolution instruments to separate isobaric compounds. ${ }^{31}$ However isomeric compounds cannot be resolved without an additional separation technique. Additionally, ion suppression effects by contaminants can drastically reduce sensitivity.

While on-line analysis overcomes most of the issues regarding sample preparation and storage, the sampling itself is still a crucial variable to control. Especially in cases where strong matrix effects are present, absolute concentrations can be difficult to obtain, as the proper addition of an internal standard is non-trivial. This becomes especially crucial in untargeted-research (biomarker discovery, etc.) where in some cases technical noise can exceed biological variability respectively it is difficult to differentiate one from the other. It is therefore of utmost importance to standardize the protocol to the highest extent. Several factors have been found to 
influence breath composition. This includes body position $^{24}$, daytime ${ }^{275}$, oral/nasal breathing ${ }^{276}$, exhalation strength $^{277}$, upper-airway restrictions ${ }^{278}$
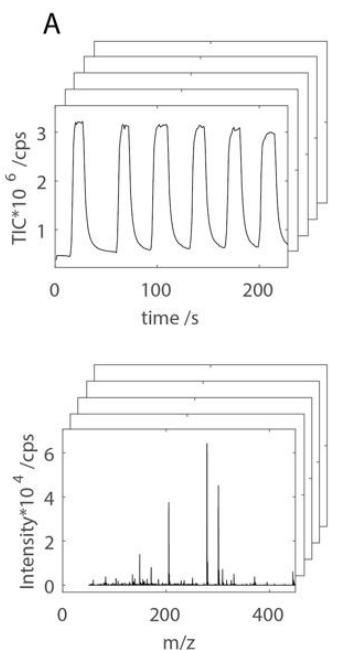

B

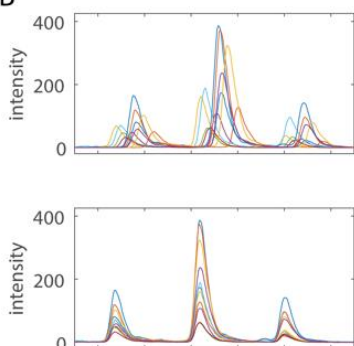

C

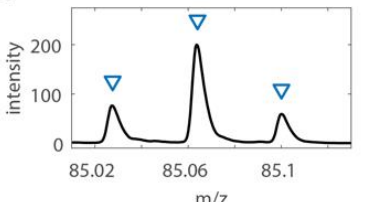

biases. $^{283}$ The medical field has established frameworks to minimize biases at all stages. However, blinding, a key measure to prevent biases,

D

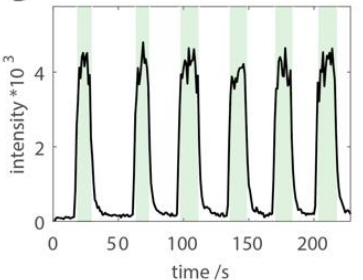

$\mathrm{E}$

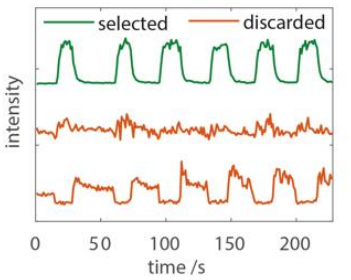

$\mathrm{F}$

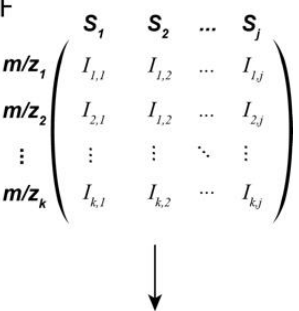

$\mathrm{G}$

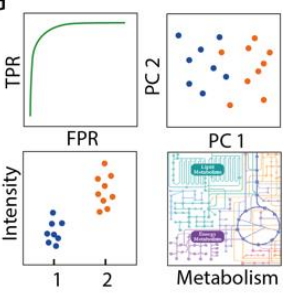

Figure 6: Scheme of a typical processing workflow for exhaled breath data obtained with mass spectrometry for on-line breath analysis. a) Conversion of the raw data into an open file format (e.g. mzXML or mzML). b) Alignment of the spectra using reference peaks. c) Peakpicking using the average spectrum of all measurements. d) Generation of time-traces and detection of timepoints of interest (green). e) Feature reduction to compounds of interest. f) Generation of the final intensity matrix by averaging the intensities during the exhalations. g) Subjection of the final matrix to the downstream statistical analysis

and even menstrual cycles $^{279}$. Even when the sampling protocol is perfectly uniform, technical variability over time can add noise to the data or lead to false discoveries when the measurements are not randomly assigned. This is usually addressed during post-processing of the data. A key-point point is data normalization, where a variety of approaches has been proposed in literature, including normalizing to an internal standard ${ }^{280}$, the total ion current (or a certain fraction of it), or peaks that are known to have very low variability in the measured system/matrix.

Batch effects can oftentimes not be corrected sufficiently using normalization only. Several supervised and unsupervised algorithms have been developed to correct for these during data analysis. ${ }^{281}$ However, when untargeted research is performed and the groups are not randomly distributed over batches, these effects can only be corrected accurately by implementing quality control samples. In the case of a lack thereof, the measurement time and/or batches should to be included as covariants. ${ }^{25}$ When using supervised methods to corrects batches while retaining group differences, careful validation approaches should be taken since these may lead to overoptimistic results in the downstream analysis. ${ }^{282}$

\subsubsection{The issue of reproducibility and the importance of external validation}

A literature search reveals a profound imbalance between the number of pilot and validation studies (see section 4.3). Overoptimistic results from discovery phases that cannot be validated externally can often be attributed to biases. The most common ones have been categorized into selection, performance, detection, attrition and reporting is not possible in discovery phase biomarker studies. Due to the vast choice of emerging data processing methods, researchers might be tempted to "try which one gives the best results". Oftentimes, this is even unavoidable if new experimental parameters have been applied and no unbiased measure of appropriate data-processing is available. However, simply comparing different model parameters or sets of biomarkers and consequently picking the best one while looking at the targeted output can yield strongly overoptimistic results, even when crossvalidations are performed. This issue has been dubbed "p-hacking" and affects all fields of highdimensional data analysis and is commonly underestimated by researchers. ${ }^{284}$ One should therefore ideally define the data analysis procedure beforehand. ${ }^{285}$ In cases where this is not possible, a certain subset of samples needs to be kept as a blind validation set until the very end,

and the results of the first un-blinded analysis thereof has to be reported.

Additionally, the lack of experimental standardization makes it difficult for researchers to compare their results across studies. Different ionization techniques result in distinct ionization efficiencies for different compound classes as well as varying influence of matrix effects. If a compound is sufficiently volatile to generate robust reference gasphase concentrations, reliable, validated, absolute concentrations could theoretically be obtained. However, most workflows to date rely on theoretical assumptions, especially in untargeted research. Therefore, future work should put strong focus on the standardization of experimental parameters as 
well as data processing workflows in order to enable large-scale multicenter validation studies.

Furthermore, cross-platform validation of on-line methods such as PTR-MS or SESI-MS with established off-line methods such as GC-MS should be pursued ${ }^{286}$ to shed more light from a method point of view on the often low reported overlap between compounds of interest for e.g. a specific disease $^{173}$.

\subsubsection{Towards standardization}

Standardization is particularly important for untargeted breath analysis studies. Recently there were recommendations proposed by Hanna et al. ${ }^{203}$. Based these recommendations, our own experience and the workflow presented in Figure 2 we recommend to take particular care of the following aspects. First, the instrument needs to be operating according to defined quality criteria (i.e. reference standards). Variability in the background due to contaminations or other environmental factors can impact sensitivity or confound specificity for certain methods (i.e. ion suppression for mass spectrometrybased methods) and should be monitored. Breath sampling at defined conditions (e.g. posture, breathing pattern) are important and need to be controlled. ${ }^{24}$

Most clinical breath analysis studies take more than a year and batch effects can occur. Regular reference gas measurements for the compounds respective compound classes of interest and in the case of untargeted studies regular breath control measurements of the operator is currently advised. This is mainly due to the lack of a suitable breath reference material capable to cover the potentially different behavior of the wide range of detected compound classes detected with some of the current on-line breath analysis methods.

If intensive testing has been performed with the goal to obtain the largest differences in a cross-sectional study then it should be followed by a validation study without changing the applied processing parameters. For this it is important that the exact parameters and thresholds used for data processing are fully described in published work. However, this is often not the case. Furthermore, the level of identification certainty for reported breath markers needs to be clearly specified to avoid data misinterpretation. Further recommendations can be found in the other parts of this section.

\subsubsection{Quantification}

Standardization and the capability of absolute quantification of metabolites in breath are crucial for robust results and therefore essential for the application of breath analysis as a diagnostic tool in clinical routine. The extent to which quantification is possible varies for different breath analysis techniques.

For SIFT-MS, the ion chemistry is understood and kinetic constants are tabulated Thus, absolute concentrations can be determined. If the ion chemistry is not fully understood, one can calibrate with standard mixtures. ${ }^{287-290}$ For accurate results, standard concentrations have to be in the same range as analyte concentrations. ${ }^{36}$ However, in complex mixtures like exhaled breath, isobaric or even isomeric interferences with other analytes are the rule rather than the exception. This can compromise accurate quantitative results.

Due to additional electric fields, ion chemistry of PTR-MS is more complicated than in SIFT-MS. However, if the ion chemistry is understood and rate constants are known, absolute concentrations can be calculated. In practice, mostly calibration is carried out with a reference gas mixture. ${ }^{81,291-295}$ However, this calibration does not account for different response factors for different species. In addition, fragmentation is an issue in PTR-MS and in order to obtain accurate quantitative results product ion distributions have to be considered for each analyte. 86123

SESI-MS, the youngest MS technique for breath analysis still lacks of possibility for absolute quantification. Since the ion chemistry is not yet understood, calibration is needed. However, many compounds that have been detected in breath using SESI-MS have very low vapor pressures (e.g. amino acids $)^{27}$. For them it is very challenging to get gaseous reference standards for calibration.

Optical methods allow for quantification since, corresponding to the Beer-Lambert law, the amount of absorbed light is proportional to the analyte concentration. However, optical methods are limited to very small molecules. ${ }^{117}$

For quantitative measurements in complex matrices such as breath, specificity is the major challenge for gas sensors. Sensors are often calibrated with pure gas standards and tested for cross-selectivity using gas mixtures, which contain the main components of exhaled breath. However, they have been rarely "calibrated" in real breath. Recently, Güntner et al. performed acetone measurements in breath using $\mathrm{Si}$ doped $\mathrm{WO}_{3}$-sensing nanoparticles and simultaneously with PTR-TOF-MS, which was calibrated beforehand with a pure acetone gas standard. Both measurements were in good agreement for this highly abundant molecule and sensor responses could be translated into concentrations using the results of PTR-TOF-MS. ${ }^{139}$

\subsection{Data analysis}

\subsubsection{Data processing workflows}

The starting point for any statistical analysis is usually an intensity matrix for all compounds of interest per subject. To obtain this from the raw data, several data-processing steps are necessary. A typical processing workflow for mass spectrometric breath data is illustrated in Figure 6. After raw data acquisition, the spectra need to be aligned. This is can be done using known reference peaks (i.e. 
omnipresent plasticizers $^{274}$ ) and recalibrating the $m / z$-axis accordingly. Afterwards, an initial peak-list needs to be generated, which is usually done by using a composite-spectrum from all measurements in untargeted studies or given externally for targeted approaches. Then, the centroided time-traces are calculated for all peaks and the target regions (i.e. exhalations, end-tidal fractions) are detected by using a known indicator for the timepoints of interest. This can be the output of an external $\mathrm{CO}_{2}$ sensor, the total ion chromatogram, or a compound that is known to be consistently present in breath, together with an appropriate algorithm. The last step is to filter only for those compounds that are higher in breath compared to background. Averaging of the selected signal intensities then yields the final intensity matrix, which can be further normalized and transformed, and subsequently subjected to statistical analysis. Different normalization approaches are available and must be chosen with caution by considering the validity of the underlying assumptions. While in PTR and SIFT, the signal intensities are often translated into absolute gasphase concentrations using theoretical models and information from different reagent ions, SESI usually corrects for varying ion source current by normalizing to the sum of signals, often after discarding the most intense ones (see also section 4.2).

The computational and instrumental developments during the last decades made it possible to analyze vast amounts of data simultaneously, leading to very high-dimensional data. ${ }^{296} \mathrm{~A}$ vast array of techniques to analyze -omics data has emerged during the last decade, which is beyond the scope of this review. The reader is therefore referred to existing literature on this topic. ${ }^{297-302}$

\subsubsection{Statistical considerations}

A number of important statistical problems hamper the progress of breath analysis in the medical field. While in some studies the lack of standardization and control for confounding factors primarily limit the reproducibility of data, the problem of false positive results (i.e. type-I-errors) has prominently emerged in the field. The focus in breath-analysis based research has shifted from a comparison of single biomarkers (e.g. nitric oxide in asthma) towards the analysis of panels of biomarkers because for most diseases the concentration of single molecules is only of limited significance. A panel of biomarkers mathematically improves predictive performance over individual markers and usually results in a more sophisticated understanding of the disease.

As a consequence, in untargeted exploratory studies the high-throughput data from the MS (approximately 1'000 - 4'000 peaks) is subject to massive statistical testing (i.e. hypothesis driven research) and the number of hypotheses tested usually outnumber the subjects included by a factor of $>100 .{ }^{111,179,194}$ This circumstance is paired with the experience that most of the peaks measured $(>99 \%)$ are usually not discriminatory for the disease of interest, i.e., the number of false hypotheses outnumber the true hypothesis in this setting by a factor of $>100 .{ }^{111,179,180,194}$ This low yield is primarily due to the fact that most signals or peaks do not correspond to a VOC or the VOCs identified are mostly of environmental origin. ${ }^{303}$ Due to the nature of the applied statistics, this mismatch produces by design a high number of type-I-errors. In other words, these are positive results which occurred just by chance (random error of data), however, they are not reproducible and possibly not generalizable to the condition tested.

One way to deal with this mismatch is to increase sample size and introduce replicate measurements (i.e. on different days) to allow estimation of the magnitude of the remaining random error and the use of formal statistical models to benchmark putative hits relative to what is expected by chance. ${ }^{304}$ However, these two approaches are often limited by available resources. Due to this, researchers often use statistical models with adjusted p-values. Simulations have shown that the false discovery rate controlling method, summarized in q-values, designed to produce the mathematically expected proportion of real biomarkers in a more balanced way compared to more conservative adjustments ${ }^{305}$, is the appropriate method. There are other methods dealing with the problem of multiple hypothesis testing (including an external validation of the initial findings), these are rarely applied in the field of breath analysis. For example, a systematic review in 2016 on exhaled-breath markers in COPD concluded that only one in 12 studies (off-line and on-line) adequately corrected for multiple hypothesis testing. ${ }^{173}$ Although all 12 studies from this review reported a successful prediction of the disease, less than $1 \%$ of the identified biomarkers candidates overlapped between the studies. ${ }^{173}$ However, the overlap might be somewhat higher when including the formation of different adducts and losses of the target molecules which can vary from instrument (e.g. commonly observed losses for PTR-MS, fragments for GC-MS or also adducts for SESI-MS). Another example is the striking imbalance between the results from all breath analysis studies on cancer $(n=63$, pooled area under the receiver operating characteristic curve 0.94, standard error 0.01) and missing successful external validation studies ${ }^{203}$ with only one recent phase-II biomarker trials. ${ }^{205}$

It has been argued that given the circumstances in which the field of breath analysis operates (i.e. small sample sizes, small effect sizes, high number of tested relationships (especially GC-MS and SESIMS), low ratio of "true" hypotheses, high inter-group flexibility in designs and processing of data) one can mathematically predict that most results in this field will be false positives. ${ }^{306}$ The problem might thus be solved by implementing the following suggestions in future studies: first, there is a need for standardization of data collection (e.g. adherence to the ISO-11843 guidelines ${ }^{204}$ ), data normalization and 
statistical analysis in order to compare and validate results from different research groups directly. Second, sample size should be adequate in relation to tested peaks (up to 10:1) and replicate measurements on different days should be done to account for random error effects. Third, besides pure untargeted hypothesis driven research, established VOCs from other bodily fluids (e.g. blood) could promote plausibility, help identifying biomarkers of interest and serve as a proof-of-principle.

Although some of the statistical issues in this field mimic the ones from genetic associations in the early $2000 \mathrm{~s}^{307}$, they can be managed reasonably and most new discoveries in this field will continue to arise from hypothesis generating research. ${ }^{306}$ The result of the statistical analysis is a set of features of interest. If these features are not carefully identified with the corresponding molecular structures a further source for reproducibility issues is created.

\subsection{Compound identification and metabolic pathways}

The identification of unknown compounds remains one of the main bottlenecks in the field of untargeted metabolomics. Out of the three types of on-line methods described in this review only mass spectrometry has the capability for identifying unknowns. High-resolution mass spectrometry methods with fragmentation capabilities (HRMS/MS) are particularly suited for this purpose $^{10}$. Other methodologies rely on a matching of the patterns of breath and standards or complementary methods (e.g., chemical sensors and PTR-MS ${ }^{129}$ ).

The steps for metabolite identification with HRMS/MS have been summarized by Schymanski et al. ${ }^{308}$. We have re-defined these five levels for online breath analysis with high-resolution mass spectrometry and complementary pre-separation mass spectrometric methods (see Figure 7).

The exact mass (level 5) of the mass feature of interest is the starting point for the identification workflow and the lowest level of identification certainty. The assignment of an unambiguous molecular formula (level 4) requires a mass spectrometer with sufficient resolving power for the mass range of interest and the selection of suitable elements and their numbers. It is particularly important to check for the formation of different adducts and losses, e.g., various adducts can be formed with SESI-MS and losses in PTR-MS can lead to data misinterpretation. The tentative structure (level 3) is based on interpretation of the fragment pattern or similarities with a database entry or the literature. The step from an unambigious formula (level 4) to a tentative structure (level 3) is the most challenging one. It is the "detective work" of figuring out the puzzle of the fragments and link them to probable molecular structures. The probable structure (level 2) based on either matching fragments or retention times with a standard, e.g. NIST matching scores higher than 800 can be considered a good spectral match ${ }^{309}$. Unequivocal compound identification respective the confirmed structure (level 1) requires a complementary preseparation method (e.g. gas chromatography or ion mobilitly for breath or liquid chromatography for breath condensates) with a sufficient high match in both dimensions. If different isomers are possible but standards not available, it is important to properly annotate them, e.g., n-pyrroline instead of 2pyrroline. This is of particular importance if the isomers are of biological relevance which will require a more thorough investigation. In some cases for molecules with very low masses $(<100 \mathrm{u})$, the use of multiple reagent ions can be used to distinguish certain compounds ${ }^{310}$. Today, structure elucidation remains to a large extent a careful manual investigation of the obtained patterns followed by confirmation measurements of pure reference standards.

A major bottleneck in the identification of breath markers is the lack of a dedicated database. Some of the most useful databases in day-to-day structure elucidation in breath analysis are the general-purpose human metabolome database (HMDB) ${ }^{311}{ }^{312}$, the largest metabolite mass spectral database Metlin ${ }^{313}$ 314 , the commercial NIST mass spectral libraries ${ }^{315}$ and mzCloud (HighChem LLC, Slovakia) ${ }^{316}$ for Orbitrap spectra. In the context of breath analysis, it is often worthwhile to also check the EPA's chemistry dashboard (Environmental Protection Agency, United States) and the microbial volatile database ${ }^{317}$. The two principal chemical databases Chemspider $^{318}$ and PubChem ${ }^{319}$ are very extensive but often result in too many possibilities to be of much use. A recent review has summarized the

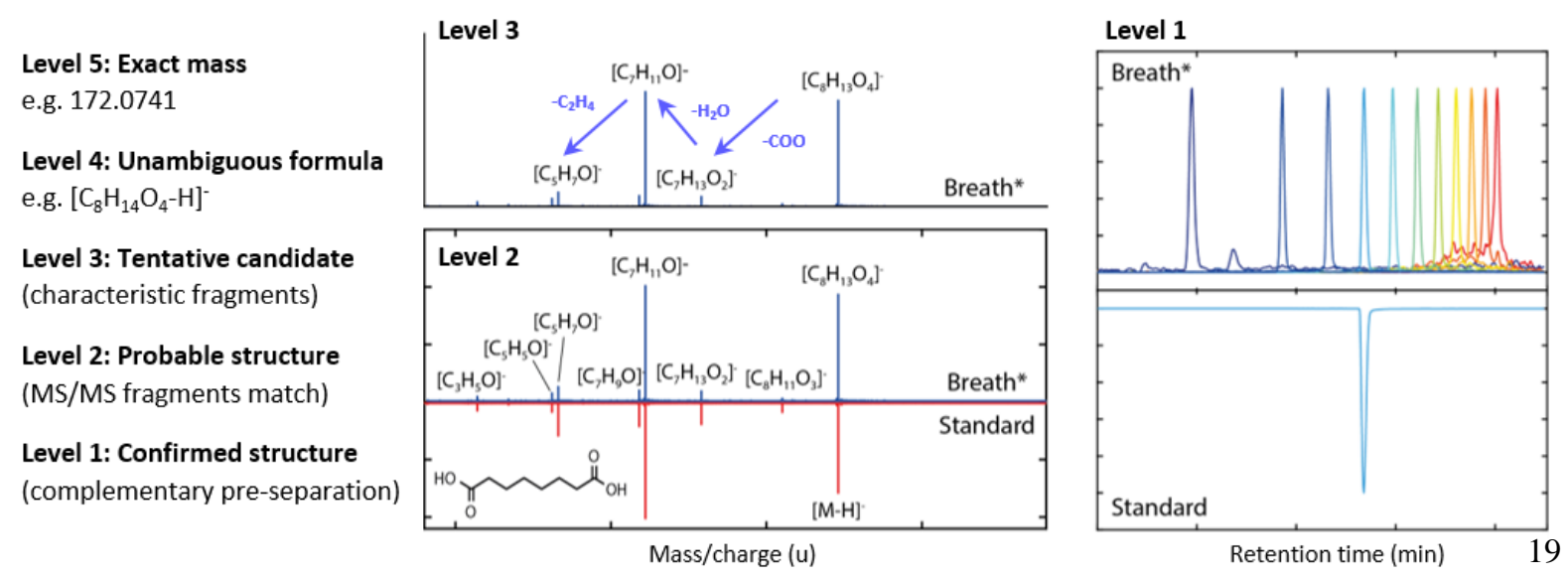

Figure 7: Levels of compound identification based on the approach by Schymanski et al. ${ }^{308}$ modified for breath analysis $(*$ the data shown here was from exhaled breath condensate analysis with UHPLC-HRMS/MS ${ }^{29}$. 
available mass spectral databases. ${ }^{320}$ Databases are useful in narrowing down likely compounds but it is important to always keep in mind the prior bias especially when working with a limited database such as HMDB compared to PubChem ${ }^{321}$. In-silica fragment MS/MS prediction can be of some use. However in many cases, there are obvious mismatches between the in-silica prediction and measured spectra, e.g., in the Metlin database. ${ }^{313} 322$ The search in the databases is useful to identify known compounds and at the same time to gain insight into metabolic pathways. A more thorough investigation of known metabolic pathways can be done by searches in the Kyoto Encyclopedia of Genes and Genomes (KEGG) database. ${ }^{323}$

\subsection{Miniaturization, portability and costs reduction}

For the application of breath analysis in clinical routine, challenges of miniaturization, portability and cost reduction need to be tackled. Chemical sensors are already miniaturized. However, as described in section 2.5 , the size of the whole sampling device is still not portable, i.e., it is often the sampling setup and the read-out electronics that dictate the overall size of the device (see Figure 5D). A setup where, for example, a sensor array is plugged into the port of a smart phone onto which a patient would breath, is thus prone to large sampling error and, at this point, science fiction.

On the other end, it is necessary that large and costly, laboratory-based instruments such as mass spectrometers are miniaturized and be made portable for breath analysis. Several portable mass spectrometers exist and have been used for field applications ${ }^{324}$, albeit very rarely for breath analysis 325. Neither the mass resolving power (usually only unit mass resolution up to several $100 \mathrm{Da}$ ) nor the sensitivity (far above $\mathrm{ppb}$ ) of miniature, fieldable mass spectrometers currently fulfill the needs of online exhaled breath analysis. The best way forward, in our opinion, is to reduce the size (and cost) of high-performance standard instruments to desktop size, and program the instrument to detect (i) accurate masses and (ii) specific MS/MS fragments of several known biomarkers for specific diseases. This "multiple reaction monitoring" (MRM) approach is well established in other fields, e.g., environmental analysis, and will provide the required chemical specificity and sensitivity of detection.

\section{Conclusions and Outlook}

Breath analysis is is attractive because it is available in nearly unlimited quantities, its analysis is noninvasive and presents no burden to the subject being measured. On-line breath analysis allows continuous monitoring of metabolic health, disease progression, and medication in short time intervals. Results are obtained immediatly which is of importance in situations were immediate action is necessary (chapter 1). There are now several research centers working in this area, dedicating a significant amount of personnel and resources, using a range of technologies, and increasingly applying them to clinical questions. Of particular promise are areas such as monitoring of disease and medications, disease phenotyping, diagnosis of inflammation and infections, novel applications, e.g. asthma diagnosis in young children, and chronobiology where continuous sampling is needed (e.g. sleep studies). Due to the wide range of detected metabolites and the sensitivity and selectivity to distinguish specific compounds in exhaled breath, the methods are expected to open a new window into the metabolism and provide additional diagnostic insight.

Technologies have developed tremendously in recent years. The most sensitive ones are mass spectrometry based, the simplest (but lower performance) ones based on chemical sensors or sensor arrays. In between in terms of cost and size are technologies such as laser spectroscopy. While technologies continue to be improved, they are still several years away of becoming mainstream for diagnosing diseases. Most applications today are proof-ofprinciple and case/control studies with a limited number of patients. There are a number of hurdles: (i) lack of standardization; (ii) determination of reliable data processing parameters, (iii) compound identification which is currently laborious and time consuming, (iv) many promising breath biomarkers that have been discovered in case-control studies still await clinical validation with large patient cohorts; and (v) the most sensitive and versatile method mass spectrometry - is not yet available as a rugged, affordable, and compact commercial instrument for clinical use. As a result of these hurdles, there is, for example, there are currently very few clinical phaseII biomarker studies for VOCs in the field of exhaled breath analysis.

In the short term we predict that exhaled breath analysis will become the method of choice for diagnosis in a small number of niche applications. An example would be diagnosing asthma in very young children and infants, who cannot perform lung function tests. It is also probable that breath analysis will play a significant role in longitudinal monitoring of disease progression, effectiveness of therapy, and adherence to medication. In the longer term, if validation studies with sufficient sample size are successful and major industries and the diagnostics sectors adopt breath-based technologies, it can easily be imagined that breath-based diagnostics will replace established tests relying on urine or blood samples, due to the ease and convenience of obtaining breath samples, and because results are available immediately.

\section{Acknowledgments}

This work is part of the Zurich Exhalomics flagship project under the umbrella of "University Medicine Zurich/Hochschulmedizin Zürich". We thank the Uniscientia Stiftung, the Schwyzer Stiftung, and the Lotte und Arnold Hotz-Sprenger Stiftung for partial 
financial support of this work.

\section{Supporting Information}

Available

The supporting information Table S1 contains the reported volatile organic compounds detected by online breath analysis methods based on a literature search. The table is grouped into 17 compound classes with the references for each compound and annotated by detection method and identification confidence. 


\section{References}

(1) Wallace, M. A. G.; Pleil, J. D. Evolution of Clinical and Environmental Health Applications of Exhaled Breath Research: Review of Methods and Instrumentation for GasPhase, Condensate, and Aerosols. Analytica Chimica Acta 2018, 1024, 18-38.

(2) Casas-Ferreira, A. M.; Nogal-Sánchez, M. d.; Pérez-Pavón, J. L.; Moreno-Cordero, B. Non-Separative Mass Spectrometry Methods for Non-Invasive Medical Diagnostics Based on Volatile Organic Compounds: A Review. Analytica Chimica Acta 2018, 1045, 10-22.

(3) Giannoukos, S.; Brkić, B.; Taylor, S.; Marshall, A.; Verbeck, G. F. Chemical Sniffing Instrumentation for Security Applications. Chemical Reviews 2016, 116, 81468172.

(4) Ahmed, W. M.; Lawal, O.; Nijsen, T. M.; Goodacre, R.; Fowler, S. J. Exhaled Volatile Organic Compounds of Infection: A Systematic Review. ACS Infectious Diseases 2017, 3, 695-710.

(5) Maciocia, G. Grundlagen Der Chinesischen Medizin; Urban \& Fischer/Elsevier 2016.

(6) Neuburger, M. Geschichte Der Medizin; F. Enke, 1906.

(7) Karamanou, M.; Androutsos, G. Antoine-Laurent De Lavoisier (1743-1794) and the Birth of Respiratory Physiology. Thorax 2013, 68, 978-979.

(8) Pauling, L.; Robinson, A. B.; Teranishi, R.; Cary, P. Proceedings of the National Academy of Sciences of the United States of America, 1971; p 2374-2376.

(9) Lawal, O.; Ahmed, W. M.; Nijsen, T. M. E.; Goodacre, R.; Fowler, S. J. Exhaled Breath Analysis: A Review of Breath-Taking Methods for Off-Line Analysis. Metabolomics 2017, 13, 1-16.

(10) Gaugg, M. T.; Bruderer, T.; Nowak, N.; Eiffert, L.; Sinues, P. M. L.; Kohler, M.; Zenobi, R. Mass-Spectrometric Detection of Omega-Oxidation Products of Aliphatic Fatty Acids in Exhaled Breath. Analytical Chemistry 2017, 89, 10329-10334.

(11) Gustafsson, L. E.; Leone, A. M.; Persson, M. G.; Wiklund, N. P.; Moncada, S. Endogenous Nitric Oxide Is Present in the Exhaled Air of Rabbits, Guinea Pigs and Humans. Biochemical and Biophysical Research Communications 1991, 181, 852-857.

(12) Dweik, R. A.; Boggs, P. B.; Erzurum, S. C.; Irvin, C. G.; Leigh, M. W.; Lundberg, J. O.; Olin, A. C.; Plummer, A. L.; Taylor, D. R.; American Thoracic Society Committee on Interpretation of Exhaled Nitric Oxide Levels for Clinical, A. An Official Ats Clinical Practice Guideline: Interpretation of Exhaled Nitric Oxide Levels (Feno) for Clinical Applications. Am J Respir Crit Care Med 2011, 184, 602-615.

(13) Simrén, M.; Stotzer, P. O. Use and Abuse of Hydrogen Breath Tests. Gut 2006, 55, 297-303.

(14) Romagnuolo, J.; Schiller, D.; Bailey, R. J. Using Breath Tests Wisely in a Gastroenterology Practice: An EvidenceBased Review of Indications and Pitfalls in Interpretation. Am J Gastroenterol 2002, 97, 1113-1126.

(15) de Lacy Costello, B.; Amann, A.; Al-Kateb, H.; Flynn, C.; Filipiak, W.; Khalid, T.; Osborne, D.; Ratcliffe, N. M. A Review of the Volatiles from the Healthy Human Body. Journal of Breath Research 2014, 8, 1-29.

(16) Simionescu, M. Cellular Components of the Air-Blood Barrier. J Cell Mol Med 2001, 5, 320-321.

(17) Martinez-Lozano Sinues, P.; Zenobi, R.; Kohler, M. Analysis of the Exhalome: A Diagnostic Tool of the Future. Chest 2013, 144, 746-749.

(18) Španěl, P.; Dryahina, K.; Smith, D. A Quantitative Study of the Influence of Inhaled Compounds on Their Concentrations in Exhaled Breath. Journal of Breath Research 2013, 7, 1-11.

(19) Pysanenko, A.; Wang, T.; Spanel, P.; Smith, D. Acetone, Butanone, Pentanone, Hexanone and Heptanone in the
Headspace of Aqueous Solution and Urine Studied by Selected Ion Flow Tube Mass Spectrometry. Rapid Communications in Mass Spectrometry 2009, 23, 10971104.

(20) Španěl, P.; Dryahina, K.; Rejšková, A.; Chippendale, T. W. E.; Smith, D. Breath Acetone Concentration; Biological Variability and the Influence of Diet. Physiological Measurement 2011, 32, N23-N31.

(21) Abbott, S. M.; Elder, J. B.; Spanel, P.; Smith, D. Quantification of Acetonitrile in Exhaled Breath and Urinary Headspace Using Selected Ion Flow Tube Mass Spectrometry. International Journal of Mass Spectrometry 2003, 228, 655-665.

(22) Garcia-Gomez, D.; Gaisl, T.; Bregy, L.; Cremonesi, A.; Sinues, P. M. L.; Kohler, M.; Zenobi, R. Real-Time Quantification of Amino Acids in the Exhalome by Secondary Electrospray Ionization-Mass Spectrometry: A Proof-of-Principle Study. Clinical Chemistry 2016, 62, 1230-1237.

(23) Blanchet, L.; Smolinska, A.; Baranska, A.; Tigchelaar, E.; Swertz, M.; Zhernakova, A.; Dallinga, J. W.; Wijmenga, C.; van Schooten, F. J. Factors That Influence the Volatile Organic Compound Content in Human Breath. Journal of Breath Research 2017, 11, 1-9.

(24) Sukul, P.; Trefz, P.; Kamysek, S.; Schubert, J. K.; Miekisch, W. Instant Effects of Changing Body Positions on Compositions of Exhaled Breath. Journal of Breath Research 2015, 9, 1-18.

(25) European Medicines Agency. Guideline on Adjustment for Baseline Covariates. https://www.ema.europa.eu/en/adjustment-baselinecovariates-clinical-trials. 01.03.2019.

(26) Garcia-Gomez, D.; Bregy, L.; Nussbaumer-Ochsner, Y.; Gaisl, T.; Kohler, M.; Zenobi, R. Detection and Quantification of Benzothiazoles in Exhaled Breath and Exhaled Breath Condensate by Real-Time Secondary Electrospray Ionization-High-Resolution Mass Spectrometry and Ultra-High Performance Liquid Chromatography. Environ Sci Technol 2015, 49, 1251912524.

(27) Garcia-Gomez, D.; Gaisl, T.; Bregy, L.; Cremonesi, A.; Sinues, P. M.; Kohler, M.; Zenobi, R. Real-Time Quantification of Amino Acids in the Exhalome by Secondary Electrospray Ionization-Mass Spectrometry: A Proof-of-Principle Study. Clin Chem 2016, 62, 1230-1237.

(28) Garcia-Gomez, D.; Martinez-Lozano Sinues, P.; BarriosCollado, C.; Vidal-de-Miguel, G.; Gaugg, M.; Zenobi, R. Identification of 2-Alkenals, 4-Hydroxy-2-Alkenals, and 4Hydroxy-2,6-Alkadienals in Exhaled Breath Condensate by Uhplc-HRMS and in Breath by Real-Time HRMS. Analytical Chemistry 2015, 87, 3087-3093.

(29) Gaugg, M. T.; Bruderer, T.; Nowak, N.; Eiffert, L.; Martinez-Lozano Sinues, P.; Kohler, M.; Zenobi, R. MassSpectrometric Detection of Omega-Oxidation Products of Aliphatic Fatty Acids in Exhaled Breath. Analytical Chemistry 2017, 89, 10329-10334.

(30) Jordan, A.; Hansel, A.; Holzinger, R.; Lindinger, W. Acetonitrile and Benzene in the Breath of Smokers and Non-Smokers Investigated by Proton Transfer Reaction Mass Spectrometry (PTR-MS). International Journal of Mass Spectrometry and Ion Processes 1995, 148, 68-70.

(31) Gaugg, M. T.; Gomez, D. G.; Barrios-Collado, C.; Vidalde-Miguel, G.; Kohler, M.; Zenobi, R.; Sinues, P. M. L. Expanding Metabolite Coverage of Real-Time Breath Analysis by Coupling a Universal Secondary Electrospray Ionization Source and High Resolution Mass Spectrometrya Pilot Study on Tobacco Smokers. Journal of Breath Research 2016, 10, 1-9.

(32) Pleil, J. D.; Kim, D.; Prah, J. D.; Rappaport, S. M. Exposure Reconstruction for Reducing Uncertainty in Risk Assessment: Example Using Mtbe Biomarkers and a Simple Pharmacokinetic Model. Biomarkers 2007, 12, 331348 
(33) Boots, A. W.; van Berkel, J. J. B. N.; Dallinga, J. W.; Smolinska, A.; Wouters, E. F.; van Schooten, F. J. The Versatile Use of Exhaled Volatile Organic Compounds in Human Health and Disease. Journal of Breath Research 2012, 6, 1-21.

(34) Smith, D.; Spanel, P. In Breath Analysis for Clinical Diagnosis and Therapeutic Monitoring; Amann, A.;Smith, D., Eds., 2005.

(35) Smith, D.; Spanel, P. Pitfalls in the Analysis of Volatile Breath Biomarkers: Suggested Solutions and SIFT-MS Quantification of Single Metabolites. Journal of Breath Research 2015, 9, 1-11.

(36) Smith, D.; Španěl, P. Status of Selected Ion Flow Tube Ms: Accomplishments and Challenges in Breath Analysis and Other Areas. Bioanalysis 2016, 8, 1183-1201.

(37) SMITH, D.; ŠPANĚL, P. In Breath Analysis for Clinical Diagnosis and Therapeutic Monitoring; WORLD SCIENTIFIC, 2005.

(38) Smith, D.; Španěl, P. Ambient Analysis of Trace Compounds in Gaseous Media by SIFT-MS. Analyst 2011, 136, 2009-2032.

(39) Španěl, P.; Smith, D. Selected Ion Flow Tube Mass Spectrometry for on-Line Trace Gas Analysis in Biology and Medicine. European Journal of Mass Spectrometry 2007, 13, 77-82.

(40) Španěl, P.; Smith, D. Progress in SIFT-MS: Breath Analysis and Other Applications. Mass Spectrometry Reviews 2011, 30, 236-267.

(41) Senthilmohan, S. T.; McEwan, M. J.; Wilson, P. F.; Milligan, D. B.; Freeman, C. G. Real Time Analysis of Breath Volatiles Using SIFT-MS in Cigarette Smoking. Redox Report 2001, 6, 185-187.

(42) Španěl, P.; Dryahina, K.; Smith, D. Acetone, Ammonia and Hydrogen Cyanide in Exhaled Breath of Several Volunteers Aged 4-83 Years. Journal of Breath Research 2007, 1, 1-4.

(43) Hryniuk, A.; Ross, B. M. Detection of Acetone and Isoprene in Human Breath Using a Combination of Thermal Desorption and Selected Ion Flow Tube Mass Spectrometry. International Journal of Mass Spectrometry 2009, 285, 26-30.

(44) Wondimu, T.; Wang, R.; Ross, B. Hydrogen Sulphide in Human Nasal Air Quantified Using Thermal Desorption and Selected Ion Flow Tube Mass Spectrometry. Journal of Breath Research 2014, 8, 1-8.

(45) Storer, M.; Salmond, J.; Dirks, K. N.; Kingham, S.; Epton, M. Mobile Selected Ion Flow Tube Mass Spectrometry (SIFT-MS) Devices and Their Use for Pollution Exposure Monitoring in Breath and Ambient Air-Pilot Study. Journal of Breath Research 2014, 8, 1-7.

(46) Španěl, P.; Dryahina, K.; Vicherková, P.; Smith, D. Increase of Methanol in Exhaled Breath Quantified by SIFT-MS Following Aspartame Ingestion. Journal of Breath Research 2015, 9, 1-7.

(47) Senthilmohan, S. T.; Milligan, D. B.; McEwan, M. J.; Freeman, C. G.; Wilson, P. F. Quantitative Analysis of Trace Gases of Breath During Exercise Using the New Sift-Ms Technique. Redox Report 2000, 5, 151-153.

(48) Smith, D.; Španěl, P.; Enderby, B.; Lenney, W.; Turner, C.; Davies, S. J. Isoprene Levels in the Exhaled Breath of 200 Healthy Pupils within the Age Range 7-18 Years Studied Using SIFT-MS. Journal of Breath Research 2010, 4, 1-7.

(49) Turner, C.; Španěl, P.; Smith, D. A Longitudinal Study of Methanol in the Exhaled Breath of 30 Healthy Volunteers Using Selected Ion Flow Tube Mass Spectrometry, SIFTMS. Physiological Measurement 2006, 27, 637-648.

(50) Turner, C.; Španěl, P.; Smith, D. A Longitudinal Study of Breath Isoprene in Healthy Volunteers Using Selected Ion Flow Tube Mass Spectrometry (SIFT-MS). Physiological Measurement 2006, 27, 13-22.

(51) Diskin, A. M.; pan 1, P.; Smith, D. Time Variation of Ammonia, Acetone, Isoprene and Ethanol in Breath: A
Quantitative SIFT-MS Study over 30 Days. Physiological Measurement 2003, 24, 107-119.

(52) Enderby, B.; Lenney, W.; Brady, M.; Emmett, C.; Španěl, P.; Smith, D. Concentrations of Some Metabolites in the Breath of Healthy Children Aged 7-18 Years Measured Using Selected Ion Flow Tube Mass Spectrometry (SIFTMS). Journal of Breath Research 2009, 3, 1-11.

(53) Španěl, P.; Davies, S.; Smith, D. Quantification of Breath Isoprene Using the Selected Ion Flow Tube Mass Spectrometric Analytical Method. Rapid Communications in Mass Spectrometry 1999, 13, 1733-1738.

(54) Španěl, P.; Wang, T.; Smith, D. Coordinated Fa-Ms and SIFT-MS Analyses of Breath Following Ingestion of D2o and Ethanol: Total Body Water, Dispersal Kinetics and Ethanol Metabolism. Physiological Measurement 2005, 26, 447-457.

(55) Španel, P.; Dryahina, K.; Smith, D. The Concentration Distributions of Some Metabolites in the Exhaled Breath of Young Adults. Journal of Breath Research 2007, 1, 1-8.

(56) Wang, T.; Pysanenko, A.; Dryahina, K.; Španěl, P.; Smith, D. Analysis of Breath, Exhaled Via the Mouth and Nose, and the Air in the Oral Cavity. Journal of Breath Research 2008, 2, 1-13.

(57) Španěl, P.; Dryahina, K.; Rejšková, A.; Chippendale, T. W. E.; Smith, D. Breath Acetone Concentration; Biological Variability and the Influence of Diet. Physiological Measurement 2011, 32, N23-N31.

(58) Turner, C.; Parekh, B.; Walton, C.; Španěl, P.; Smith, D.; Evans, M. An Exploratory Comparative Study of Volatile Compounds in Exhaled Breath and Emitted by Skin Using Selected Ion Flow Tube Mass Spectrometry. Rapid Communications in Mass Spectrometry 2008, 22, 526-532.

(59) Wilson, P. F.; Freeman, C. G.; McEwan, M. J.; Milligan, D. B.; Allardyce, R. A.; Shaw, G. M. In Situ Analysis of Solvents on Breath and Blood: A Selected Ion Flow Tube Mass Spectrometric Study. Rapid Communications in Mass Spectrometry 2002, 16, 427-432.

(60) Smith, D.; Wang, T.; Spanel, P. On-Line, Simultaneous Quantification of Ethanol, Some Metabolites and Water Vapour in Breath Following the Ingestion of Alcohol. Physiological Measurement 2002, 23, 477-489.

(61) Bloor, R. N.; Španěl, P.; Smith, D. Quantification of Breath Carbon Disulphide and Acetone Following a Single Dose of Disulfiram (Antabuse) Using Selected Ion Flow Tube Mass Spectrometry (SIFT-MS). Addiction Biology 2006, 11, 163-169.

(62) Davies, S.; Španel, P.; Smith, D. A New 'Online' Method to Measure Increased Exhaled Isoprene in End-Stage Renal Failure. Nephrology Dialysis Transplantation 2001, 16, 836-839.

(63) Boshier, P. R.; Cushnir, J. R.; Mistry, V.; Knaggs, A.; Španěl, P.; Smith, D.; Hanna, G. B. On-Line, Real Time Monitoring of Exhaled Trace Gases by SIFT-MS in the Perioperative Setting: A Feasibility Study. The Analyst 2011, 136, 3233-3237.

(64) Storer, M.; Dummer, J.; Lunt, H.; Scotter, J.; McCartin, F.; Cook, J.; Swanney, M.; Kendall, D.; Logan, F.; Epton, M. Measurement of Breath Acetone Concentrations by Selected Ion Flow Tube Mass Spectrometry in Type 2 Diabetes. Journal of Breath Research 2011, 5, 1-5.

(65) Endre, Z. H.; Pickering, J. W.; Storer, M. K.; Hu, W.-P.; Moorhead, K. T.; Allardyce, R.; McGregor, D. O.; Scotter, J. M. Breath Ammonia and Trimethylamine Allow RealTime Monitoring of Haemodialysis Efficacy. Physiological Measurement 2011, 32, 115-130.

(66) Shestivska, V.; Nemec, A.; Dřevínek, P.; Sovová, K.; Dryahina, K.; Španěl, P. Quantification of Methyl Thiocyanate in the Headspace of Pseudomonas Aeruginosa Cultures and in the Breath of Cystic Fibrosis Patients by Selected Ion Flow Tube Mass Spectrometry. Rapid Communications in Mass Spectrometry 2011, 25, 24592467. 
(67) Dryahina, K.; Španěl, P.; Pospíšilová, V.; Sovová, K.; Hrdlička, L.; Machková, N.; Lukáš, M.; Smith, D. Quantification of Pentane in Exhaled Breath, a Potential Biomarker of Bowel Disease, Using Selected Ion Flow Tube Mass Spectrometry. Rapid Communications in Mass Spectrometry 2013, 27, 1983-1992.

(68) Dryahina, K.; Pospíšilová, V.; Sovová, K.; Shestivska, V.; Kubišta, J.; Spesyvyi, A.; Pehal, F.; Turzíková, J.; Votruba, J.; Španěl, P. Exhaled Breath Concentrations of Acetic Acid Vapour in Gastro-Esophageal Reflux Disease. Journal of Breath Research 2014, 8, 1-6.

(69) Wang, M. H.; Chong, K. C.; Storer, M.; Pickering, J. W.; Endre, Z. H.; Lau, S. Y.; Kwok, C.; Lai, M.; Chung, H. Y.; Ying Zee, B. C. Use of a Least Absolute Shrinkage and Selection Operator (Lasso) Model to Selected Ion Flow Tube Mass Spectrometry (SIFT-MS) Analysis of Exhaled Breath to Predict the Efficacy of Dialysis: A Pilot Study. Journal of Breath Research 2016, 10, 1-7.

(70) Storer, M.; Dummer, J.; Lunt, H.; Scotter, J.; McCartin, F.; Cook, J.; Swanney, M.; Kendall, D.; Logan, F.; Epton, M. Measurement of Breath Acetone Concentrations by Selected Ion Flow Tube Mass Spectrometry in Type 2 Diabetes. Journal of Breath Research 2011, 5.

(71) Adams, N. G.; Smith, D. The Selected Ion Flow Tube (Sift); a Technique for Studying Ion-Neutral Reactions. International Journal of Mass Spectrometry and Ion Physics 1976, 21, 349-359.

(72) Spanel, P.; Smith, D. On-Line Measurement of the Absolute Humidity of Air, Breath and Liquid Headspace Samples by Selected Ion Flow Tube Mass Spectrometry. Rapid Communications in Mass Spectrometry 2001, 15, 563-569.

(73) Ross, B. M. Sub-Parts Per Billion Detection of Trace Volatile Chemicals in Human Breath Using Selected Ion Flow Tube Mass Spectrometry. BMC Res Notes 2008, 1, 15 .

(74) Pysanenko, A.; Španěl, P.; Smith, D. Analysis of the Isobaric Compounds Propanol, Acetic Acid and Methyl Formate in Humid Air and Breath by Selected Ion Flow Tube Mass Spectrometry, SIFT-MS. International Journal of Mass Spectrometry 2009, 285, 42-48.

(75) Španěl, P.; Ji, Y.; Smith, D. Sift Studies of the Reactions of $\mathrm{H} 3 \mathrm{O}+$, No+ and $\mathrm{O} 2+$ with a Series of Aldehydes and Ketones. International Journal of Mass Spectrometry and Ion Processes 1997, 165-166, 25-37.

(76) Spesyvyi, A.; Smith, D.; Spanel, P. Selected Ion Flow-Drift Tube Mass Spectrometry: Quantification of Volatile Compounds in Air and Breath. Analytical Chemistry 2015, 87, 12151-12160.

(77) Ferguson, E. E.; Fehsenfeld, F. C.; Schmeltekopf, A. L. In Advances in Atomic and Molecular Physics; Bates, D. R.;Estermann, I., Eds.; Academic Press, 1969; Vol. 5.

(78) Lagg, A.; Taucher, J.; Hansel, A.; Lindinger, W. Applications of Proton Transfer Reactions to Gas Analysis. International Journal of Mass Spectrometry and Ion Processes 1994, 134, 55-66.

(79) Hansel, A.; Jordan, A.; Holzinger, R.; Prazeller, P.; Vogel, W.; Lindinger, W. Proton Transfer Reaction Mass Spectrometry: On-Line Trace Gas Analysis at the Ppb Level. International Journal of Mass Spectrometry and Ion Processes 1995, 149-150, 609-619.

(80) Harrison, G. R.; Critchley, A. D. J.; Mayhew, C. A.; Thompson, J. M. Real-Time Breath Monitoring of Propofol and Its Volatile Metabolites During Surgery Using a Novel Mass Spectrometric Technique: A Feasibility Study. British Journal of Anaesthesia 2003, 91, 797-799.

(81) Herbig, J.; Muller, M.; Schallhart, S.; Titzmann, T.; Graus, M.; Hansel, A. On-Line Breath Analysis with Ptr-Tof. Journal of Breath Research 2009, 3, 1-10.

(82) Trefz, P.; Schmidt, M.; Oertel, P.; Obermeier, J.; Brock, B.; Kamysek, S.; Dunk1, J.; Zimmermann, R.; Schubert, J. K.; Miekisch, W. Continuous Real Time Breath Gas Monitoring in the Clinical Environment by Proton-
Transfer-Reaction-Time-of-Flight-Mass Spectrometry. Analytical Chemistry 2013, 85, 10321-10329.

(83) Lindinger, W.; Hansel, A.; Jordan, A. On-Line Monitoring of Volatile Organic Compounds at Pptv Levels by Means of Proton-Transfer-Reaction Mass Spectrometry (PTR-MS) Medical Applications, Food Control and Environmental Research. International Journal of Mass Spectrometry 1998, 173, 191-241.

(84) Lindinger, W.; Hansel, A.; Jordan, A. Proton-TransferReaction Mass Spectrometry (PTR-MS): On-Line Monitoring of Volatile Organic Compounds at Pptv Levels. Chemical Society Reviews 1998, 27, 347-354.

(85) Blake, R. S.; Monks, P. S.; Ellis, A. M. Proton-Transfer Reaction Mass Spectrometry. Chemical Reviews 2009, 109, 861-896.

(86) Kari, E.; Miettinen, P.; Yli-Pirilä, P.; Virtanen, A.; Faiola, C. L. Ptr-Tof-Ms Product Ion Distributions and HumidityDependence of Biogenic Volatile Organic Compounds. International Journal of Mass Spectrometry 2018, 430, 8797.

(87) Jordan, A.; Haidacher, S.; Hanel, G.; Hartungen, E.; Herbig, J.; Märk, L.; Schottkowsky, R.; Seehauser, H.; Sulzer, P.; Märk, T. D. An Online Ultra-High Sensitivity Proton-Transfer-Reaction Mass-Spectrometer Combined with Switchable Reagent Ion Capability (Ptr+Sri-Ms). International Journal of Mass Spectrometry 2009, 286, 3238.

(88) Sulzer, P.; Edtbauer, A.; Hartungen, E.; Jürschik, S.; Jordan, A.; Hanel, G.; Feil, S.; Jaksch, S.; Märk, L.; Märk, T. D. From Conventional Proton-Transfer-Reaction Mass Spectrometry (PTR-MS) to Universal Trace Gas Analysis. International Journal of Mass Spectrometry 2012, 321-322, 66-70.

(89) Prazeller, P.; Palmer, P. T.; Boscaini, E.; Jobson, T.; Alexander, M. Proton Transfer Reaction Ion Trap Mass Spectrometer. Rapid Communications in Mass Spectrometry 2003, 17, 1593-1599.

(90) O'Hara, M. E.; Fernández Del Río, R.; Holt, A.; Pemberton, P.; Shah, T.; Whitehouse, T.; Mayhew, C. A. Limonene in Exhaled Breath Is Elevated in Hepatic Encephalopathy. Journal of Breath Research 2016, 10, 1-11.

(91) Krechmer, J.; Lopez-Hilfiker, F.; Koss, A.; Hutterli, M.; Stoermer, C.; Deming, B.; Kimmel, J.; Warneke, C.; Holzinger, R.; Jayne, J. et al. Evaluation of a New ReagentIon Source and Focusing Ion-Molecule Reactor for Use in Proton-Transfer-Reaction Mass Spectrometry. Analytical Chemistry 2018, 90, 12011-12018.

(92) Chen, Y. H.; Hill, H. H.; Wittmer, D. P. Analytical Merit of Electrospray Ion Mobility Spectrometry as a Chromatographic Detector. Journal of Microcolumn Separations 1994, 6, 515-524.

(93) Fuerstenau S., K. P., Fenn J. B. In: Proceedings

of the 34th Asms Conference on Mass Spectrometry and Allied Topics. Proceedings of the 47th ASMS Conference on Mass Spectrometry, Dallas, TX 1999, 507-507.

(94) Whitehouse C. M. , L. F., Meng C. K. , Fenn J. B. American Society for Mass Spectrometry (ASMS) Conference, Ohio, United States, 1968; p 507-507.

(95) Wu, C.; Siems, W. F.; Hill, H. H. Secondary Electrospray Ionization Ion Mobility Spectrometry/Mass Spectrometry of Illicit Drugs. Analytical Chemistry 2000, 72, 396-403.

(96) Sinues, P. M. L.; Criado, E.; Vidal, G. Mechanistic Study on the Ionization of Trace Gases by an Electrospray Plume. International Journal of Mass Spectrometry 2012, 313, 2129.

(97) Vidal-de-Miguel, G.; Herrero, A. Secondary Electrospray Ionization of Complex Vapor Mixtures. Theoretical and Experimental Approach. J Am Soc Mass Spectrom 2012, 23, 1085-1096.

(98) Rioseras, A. T.; Gaugg, M. T.; Sinues, P. M. L. Secondary Electrospray Ionization Proceeds Via Gas-Phase Chemical Ionization. Analytical Methods 2017, 9, 5052-5057. 
(99) Martinez-Lozano, P.; Fernandez de la Mora, J. Direct Analysis of Fatty Acid Vapors in Breath by Electrospray Ionization and Atmospheric Pressure Ionization-Mass Spectrometry. Analytical Chemistry 2008, 80, 8210-8215.

(100) Chen, H. W.; Sun, Y. P.; Wortmann, A.; Gu, H. W.; Zenobi, R. Differentiation of Maturity and Quality of Fruit Using Noninvasive Extractive Electrospray Ionization Quadrupole Time-of-Flight Mass Spectrometry. Analytical Chemistry 2007, 79, 1447-1455.

(101) Tejero Rioseras, A.; Singh, K. D.; Nowak, N.; Gaugg, M. T.; Bruderer, T.; Zenobi, R.; Sinues, P. M. Real-Time Monitoring of Tricarboxylic Acid Metabolites in Exhaled Breath. Analytical Chemistry 2018, 90, 6453-6460.

(102) Tam, M.; Hill, H. H., Jr. Secondary Electrospray Ionization-Ion Mobility Spectrometry for Explosive Vapor Detection. Analytical Chemistry 2004, 76, 2741-2747.

(103) Chen, H.; Wortmann, A.; Zhang, W.; Zenobi, R. Rapid in Vivo Fingerprinting of Nonvolatile Compounds in Breath by Extractive Electrospray Ionization Quadrupole Time-ofFlight Mass Spectrometry. Angew Chem Int Ed Engl 2007, $46,580-583$.

(104) Zamora, D.; Amo-Gonzalez, M.; Lanza, M.; de la Mora, G. F.; de la Mora, J. F. Reaching a Vapor Sensitivity of 0.01 Parts Per Quadrillion in the Screening of Large Volume Freight. Analytical Chemistry 2018, 90, 2468-2474.

(105) Steiner, W. E.; Clowers, B. H.; Haigh, P. E.; Hill, H. H. Secondary Ionization of Chemical Warfare Agent Simulants: Atmospheric Pressure Ion Mobility Time-ofFlight Mass Spectrometry. Analytical Chemistry 2003, 75, 6068-6076.

(106) Martinez-Lozano, P.; Rus, J.; de la Mora, G. F.; Hernandez, M.; de la Mora, J. F. Secondary Electrospray Ionization (Sesi) of Ambient Vapors for Explosive Detection at Concentrations Below Parts Per Trillion. Journal of the American Society for Mass Spectrometry 2009, 20, 287294.

(107) Chen, H.; Yang, S.; Wortmann, A.; Zenobi, R. Neutral Desorption Sampling of Living Objects for Rapid Analysis by Extractive Electrospray Ionization Mass Spectrometry. Angew Chem Int Ed Engl 2007, 46, 7591-7594.

(108) Liang, J. C.; Hang, Y. P.; Chingin, K.; Hu, L. H.; Chen, H. W. Rapid Differentiation of Microbial Cultures Based on the Analysis of Headspace Volatiles by Atmospheric Pressure Chemical Ionization Mass Spectrometry. Rsc Advances 2014, 4, 25326-25329.

(109) Martinez-Lozano, P.; de la Mora, J. F. Electrospray Ionization of Volatiles in Breath. International Journal of Mass Spectrometry 2007, 265, 68-72.

(110) Sinues, P. M.; Kohler, M.; Zenobi, R. Monitoring Diurnal Changes in Exhaled Human Breath. Analytical Chemistry 2013, 85, 369-373.

(111) Schwarz, E. I.; Martinez-Lozano Sinues, P.; Bregy, L.; Gaisl, T.; Garcia Gomez, D.; Gaugg, M. T.; Suter, Y.; Stebler, N.; Nussbaumer-Ochsner, Y.; Bloch, K. E. et al. Effects of Cpap Therapy Withdrawal on Exhaled Breath Pattern in Obstructive Sleep Apnoea. Thorax 2016, 71, 110117.

(112) Gaugg, M. T.; Engler, A.; Nussbaumer-Ochsner, Y.; Bregy, L.; Stoberl, A. S.; Gaisl, T.; Bruderer, T.; Zenobi, R.; Kohler, M.; Martinez-Lozano Sinues, P. Metabolic Effects of Inhaled Salbutamol Determined by Exhaled Breath Analysis. Journal of Breath Research 2017, 11, 1-11.

(113) Singh, K. D.; Del Miguel, G. V.; Gaugg, M. T.; Ibanez, A. J.; Zenobi, R.; Kohler, M.; Frey, U.; Sinues, P. M. Translating Secondary Electrospray Ionization-HighResolution Mass Spectrometry to the Clinical Environment. Journal of Breath Research 2018, 12, 1-10.

(114) Wang, C.; Sahay, P. Breath Analysis Using Laser Spectroscopic Techniques: Breath Biomarkers, Spectral Fingerprints, and Detection Limits. Sensors 2009, 9, 82308262 .

(115) Bayrakli, I. Breath Analysis Using External Cavity Diode Lasers: A Review. J Biomed Opt 2017, 22, 1-15.
(116) Henderson, B.; Khodabakhsh, A.; Metsala, M.; Ventrillard, I.; Schmidt, F. M.; Romanini, D.; Ritchie, G. A. D.; Hekkert, S. T.; Briot, R.; Risby, T. et al. Laser Spectroscopy for Breath Analysis: Towards Clinical Implementation. Applied Physics B-Lasers and Optics 2018, 124, 1-21.

(117) Metsälä, M. Optical Techniques for Breath Analysis: From Single to Multi-Species Detection. Journal of Breath Research 2018, 12, 1-6.

(118) Chow, K.; Short, M.; Lam, S.; McWilliams, A.; Zeng, H. A Raman Cell Based on Hollow Core Photonic Crystal Fiber

for Human Breath Analysis. Med. Phys. 2014, 41, 1-10.

(119) Chen, Y.; Zhang, Y.; Pan, F.; Liu, J.; Wang, K.; Zhang, C.; Cheng, S.; Lu, L.; Zhang, W.; Zhang, Z. et al. Breath Analysis Based on Surface-Enhanced Raman Scattering Sensors Distinguishes Early and Advanced Gastric Cancer Patients from Healthy Persons. ACS Nano 2016, 10, 81698179.

(120) Gustafsson, L. E. In Breath Analysis for Clinical Diagnosis and Therapeutic Monitoring; WORLD SCIENTIFIC, 2005.

(121) Heinrich, K.; Fritsch, T.; Hering, P.; Muertz, M. Infrared Laser-Spectroscopic Analysis of (No)-N-14 and (No)-N-15 in Human Breath. Applied Physics B-Lasers and Optics 2009, 95, 281-286.

(122) Maksim. Atmosfaerisk_spredning. https://commons.wikimedia.org/wiki/File:Atmosfaerisk_spr edning.png. 01.01.2019.

(123) Hundt, P. M.; Muller, M.; Mangold, M.; Tuzson, B.; Scheidegger, P.; Looser, H.; Huglin, C.; Emmenegger, L. Mid-Ir Spectrometer for Mobile, Real-Time Urban No2 Measurements. Atmospheric Measurement Techniques 2018, 11, 2669-2681.

(124) Sun, M.; Jiang, C.; Gong, Z.; Zhao, X.; Chen, Z.; Wang, Z.; Kang, M.; Li, Y.; Wang, C. A Fully Integrated Standalone Portable Cavity Ringdown Breath Acetone Analyzer. Rev Sci Instrum 2015, 86, 1-9.

(125) Güntner, A. T.; Pineau, N. J.; Mochalski, P.; Wiesenhofer, H.; Agapiou, A.; Mayhew, C. A.; Pratsinis, S. E. Sniffing Entrapped Humans with Sensor Arrays. Analytical Chemistry 2018, 90, 4940-4945.

(126) Mastrigt, E.; Reyes-Reyes, A.; Brand, K.; Bhattacharya, N.; Urbach, H. P.; Stubbs, A. P.; de Jongste, J. C.; Pijnenburg, M. W. Exhaled Breath Profiling Using Broadband Quantum Cascade Laser-Based Spectroscopy in Healthy Children and Children with Asthma and Cystic Fibrosis. Journal of Breath Research 2016, 10, 1-8.

(127) Di Natale, C.; Paolesse, R.; Martinelli, E.; Capuano, R. Solid-State Gas Sensors for Breath Analysis: A Review. Analytica Chimica Acta 2014, 824, 1-17.

(128) Mädler, L.; Roessler, A.; Pratsinis, S. E.; Sahm, T.; Gurlo, A.; Barsan, N.; Weimar, U. Direct Formation of Highly Porous Gas-Sensing Films by in Situ Thermophoretic Deposition of Flame-Made Pt/Sno2 Nanoparticles. Sensors and Actuators B: Chemical 2006, 114, 283-295.

(129) Hibbard, T.; Crowley, K.; Kelly, F.; Ward, F.; Holian, J.; Watson, A.; Killard, A. J. Point of Care Monitoring of Hemodialysis Patients with a Breath Ammonia Measurement Device Based on Printed Polyaniline Nanoparticle Sensors. Analytical Chemistry 2013, 85, 12158-12165.

(130) McQuade, D. T.; Pullen, A. E.; Swager, T. M. Conjugated Polymer-Based Chemical Sensors. Chemical Reviews 2000, 100, 2537-2574.

(131) Shao, Y.; Wang, J.; Wu, H.; Liu, J.; Aksay, I. A.; Lin, Y. Graphene Based Electrochemical Sensors and Biosensors: A Review. Electroanalysis 2010, 22, 1027-1036.

(132) Schroeder, V.; Savagatrup, S.; He, M.; Lin, S.; Swager, T. M. Carbon Nanotube Chemical Sensors. Chemical Reviews 2019, 119, 599-663.

(133) Hübert, T.; Boon-Brett, L.; Black, G.; Banach, U. Hydrogen Sensors - a Review. Sensors and Actuators B: Chemical 2011, 157, 329-352. 
(134) de Lacy Costello, B. P. J.; Ledochowski, M.; Ratcliffe, N. M. The Importance of Methane Breath Testing: A Review. Journal of Breath Research 2013, 7, 1-8.

(135) Bedioui, F.; Villeneuve, N. Electrochemical Nitric Oxide Sensors for Biological Samples - Principle, Selected Examples and Applications. Electroanalysis 2003, 15, 5-18.

(136) Iverson, M. N.; Hofferber, M. E.; Stapleton, A. J. Nitric Oxide Sensors for Biological Applications. Chemosensors 2018, 6, 1-13.

(137) Neelamegan, R.; Saka, V.; Tamilarasu, K.; Rajaram, M.; Selvarajan, S.; Chandrasekaran, A. Clinical Utility of Fractional Exhaled Nitric Oxide (Feno) as a Biomarker to Predict Severity of Disease and Response to Inhaled Corticosteroid (Ics) in Asthma Patients. J Clin Diagn Res 2016, 10, FC01-FC06.

(138) Righettoni, M.; Tricoli, A.; Pratsinis, S. E. Si:Wo3 Sensors for Highly Selective Detection of Acetone for Easy Diagnosis of Diabetes by Breath Analysis. Analytical Chemistry 2010, 82, 3581-3587.

(139) Güntner, A. T.; Sievi, N. A.; Theodore, S. J.; Gulich, T.; Kohler, M.; Pratsinis, S. E. Noninvasive Body Fat Burn Monitoring from Exhaled Acetone with Si-Doped Wo3Sensing Nanoparticles. Analytical Chemistry 2017, 89, 10578-10584.

(140) Güntner, A. T.; Righettoni, M.; Pratsinis, S. E. Selective Sensing of Nh3 by Si-Doped A-Moo3 for Breath Analysis. Sensors and Actuators B: Chemical 2016, 223, 266-273.

(141) Das, S.; Pal, S.; Mitra, M. Significance of Exhaled Breath Test in Clinical Diagnosis: A Special Focus on the Detection of Diabetes Mellitus. Journal of medical and biological engineering 2016, 36, 605-624.

(142) Akamatsu, T.; Itoh, T.; Shin, W. Mixed-Potential Gas Sensors Using an Electrolyte Consisting of Zinc Phosphate Glass and Benzimidazole. Sensors 2017, 17, 1-8.

(143) Woo, H.-S.; Kwak, C.-H.; Kim, I.-D.; Lee, J.-H. Selective, Sensitive, and Reversible Detection of H2s Using MoDoped Zno Nanowire Network Sensors. Journal of Materials Chemistry A 2014, 2, 6412-6418.

(144) Donald, M. J.; Paterson, B. End Tidal Carbon Dioxide Monitoring in Prehospital and Retrieval Medicine: A Review. Emergency medicine journal : EMJ 2006, 23, 728730.

(145) Güntner, A. T.; Pineau, N. J.; Chie, D.; Krumeich, F.; Pratsinis, S. E. Selective Sensing of Isoprene by Ti-Doped Zno for Breath Diagnostics. Journal of Materials Chemistry B 2016, 4, 5358-5366.

(146) Obermeier, J.; Trefz, P.; Wex, K.; Sabel, B.; Schubert, J. K.; Miekisch, W. Electrochemical Sensor System for Breath Analysis of Aldehydes, Co and No. Journal of Breath Research 2015, 9, 1-10.

(147) Schon, S.; Theodore, S. J.; Güntner, A. T. Versatile Breath Sampler for Online Gas Sensor Analysis. Sensors and Actuators B: Chemical 2018, 273, 1780-1785.

(148) van den Broek, J.; Güntner, A. T.; Pratsinis, S. E. Highly Selective and Rapid Breath Isoprene Sensing Enabled by Activated Alumina Filter. ACS Sensors 2018, 3, 677-683.

(149) Gelperin, A.; Johnson, A. T. C. Nanotube-Based Sensor Arrays for Clinical Breath Analysis. Journal of Breath Research 2008, 2, 1-6.

(150) Konvalina, G.; Haick, H. Sensors for Breath Testing: From Nanomaterials to Comprehensive Disease Detection. Accounts of Chemical Research 2014, 47, 66-76.

(151) Li, W.; Liu, H. Y.; Xie, D. D.; He, Z. C.; Pi, X. T. Lung Cancer Screening Based on Type-Different Sensor Arrays. Scientific Reports 2017, 7, 1-12.

(152) Rioseras, A. T.; Singh, K. D.; Nowak, N.; Gaugg, M. T.; Bruderer, T.; Zenobi, R.; Sinues, P. M. L. Real-Time Monitoring of Tricarboxylic Acid Metabolites in Exhaled Breath. Analytical Chemistry 2018, 90, 6453-6460.

(153) King, J.; Kupferthaler, A.; Frauscher, B.; Hackner, H.; Unterkofler, K.; Teschl, G.; Hinterhuber, H.; Amann, A.; Hogl, B. Measurement of Endogenous Acetone and
Isoprene in Exhaled Breath During Sleep. Physiol Meas 2012, 33, 413-428.

(154) King, J.; Koc, H.; Unterkofler, K.; Mochalski, P.; Kupferthaler, A.; Teschl, G.; Teschl, S.; Hinterhuber, H.; Amann, A. Physiological Modeling of Isoprene Dynamics in Exhaled Breath. Journal of Theoretical Biology 2010, 267, 626-637.

(155) King, J.; Kupferthaler, A.; Unterkofler, K.; Koc, H.; Teschl, S.; Teschl, G.; Miekisch, W.; Schubert, J.; Hinterhuber, H.; Amann, A. Isoprene and Acetone Concentration Profiles During Exercise on an Ergometer. Journal of Breath Research 2009, 3, 1-16.

(156) Ferrante, G.; La Grutta, S. The Burden of Pediatric Asthma. Front Pediatr 2018, 6, 1-7.

(157) Guilbert, T. W.; Morgan, W. J.; Zeiger, R. S.; Mauger, D. T.; Boehmer, S. J.; Szefler, S. J.; Bacharier, L. B.; Lemanske, R. F., Jr.; Strunk, R. C.; Allen, D. B. et al. Long-Term Inhaled Corticosteroids in Preschool Children at High Risk for Asthma. N Engl J Med 2006, 354, 19851997.

(158) Pedersen, S. E.; Hurd, S. S.; Lemanske, R. F., Jr.; Becker, A.; Zar, H. J.; Sly, P. D.; Soto-Quiroz, M.; Wong, G.; Bateman, E. D.; Global Initiative for, A. Global Strategy for the Diagnosis and Management of Asthma in Children 5 Years and Younger. Pediatr Pulmonol 2011, 46, 1-17.

(159) Lane, C.; Knight, D.; Burgess, S.; Franklin, P.; Horak, F.; Legg, J.; Moeller, A.; Stick, S. Epithelial Inducible Nitric Oxide Synthase Activity Is the Major Determinant of Nitric Oxide Concentration in Exhaled Breath. Thorax 2004, 59, 757-760.

(160) Taylor, D. R.; Pijnenburg, M. W.; Smith, A. D.; De Jongste, J. C. Exhaled Nitric Oxide Measurements: Clinical Application and Interpretation. Thorax 2006, 61, 817-827.

(161) Moeller, A.; Diefenbacher, C.; Lehmann, A.; Rochat, M.; Brooks-Wildhaber, J.; Hall, G. L.; Wildhaber, J. H. Exhaled Nitric Oxide Distinguishes between Subgroups of Preschool Children with Respiratory Symptoms. Journal of Allergy and Clinical Immunology 2008, 121, 705-709.

(162) Singer, F.; Luchsinger, I.; Inci, D.; Knauer, N.; Latzin, P.; Wildhaber, J. H.; Moeller, A. Exhaled Nitric Oxide in Symptomatic Children at Preschool Age Predicts Later Asthma. Allergy 2013, 68, 531-538.

(163) Caudri, D.; Wijga, A. H.; Hoekstra, M. O.; Kerkhof, M.; Koppelman, G. H.; Brunekreef, B.; Smit, H. A.; de Jongste, J. C. Prediction of Asthma in Symptomatic Preschool Children Using Exhaled Nitric Oxide, Rint and Specific Ige. Thorax 2010, 65, 801-807.

(164) Neerincx, A. H.; Vijverberg, S. J. H.; Bos, L. D. J.; Brinkman, P.; van der Schee, M. P.; de Vries, R.; Sterk, P. J.; Maitland-van der Zee, A. H. Breathomics from Exhaled Volatile Organic Compounds in Pediatric Asthma. Pediatr Pulmonol 2017, 52, 1616-1627.

(165) Caldeira, M.; Perestrelo, R.; Barros, A. S.; Bilelo, M. J.; Morete, A.; Camara, J. S.; Rocha, S. M. Allergic Asthma Exhaled Breath Metabolome: A Challenge for Comprehensive Two-Dimensional Gas Chromatography. $J$ Chromatogr A 2012, 1254, 87-97.

(166) Dallinga, J. W.; Robroeks, C. M.; van Berkel, J. J.; Moonen, E. J.; Godschalk, R. W.; Jobsis, Q.; Dompeling, E.; Wouters, E. F.; van Schooten, F. J. Volatile Organic Compounds in Exhaled Breath as a Diagnostic Tool for Asthma in Children. Clin Exp Allergy 2010, 40, 68-76.

(167) Gahleitner, F.; Guallar-Hoyas, C.; Beardsmore, C. S.; Pandya, H. C.; Thomas, C. P. Metabolomics Pilot Study to Identify Volatile Organic Compound Markers of Childhood Asthma in Exhaled Breath. Bioanalysis 2013, 5, 2239-2247.

(168) Meyer, N.; Dallinga, J. W.; Nuss, S. J.; Moonen, E. J.; van Berkel, J. J.; Akdis, C.; van Schooten, F. J.; Menz, G. Defining Adult Asthma Endotypes by Clinical Features and Patterns of Volatile Organic Compounds in Exhaled Air. Respir Res 2014, 15, 1-8.

(169) Ibrahim, B.; Basanta, M.; Cadden, P.; Singh, D.; Douce, D.; Woodcock, A.; Fowler, S. J. Non-Invasive Phenotyping 
Using Exhaled Volatile Organic Compounds in Asthma. Thorax 2011, 66, 804-809.

(170) Brinkman, P.; van de Pol, M. A.; Gerritsen, M. G.; Bos, L. D.; Dekker, T.; Smids, B. S.; Sinha, A.; Majoor, C. J.; Sneeboer, M. M.; Knobel, H. H. et al. Exhaled Breath Profiles in the Monitoring of Loss of Control and Clinical Recovery in Asthma. Clin Exp Allergy 2017, 47, 11591169.

(171) Brode, S. K.; Ling, S. C.; Chapman, K. R. Alpha-1 Antitrypsin Deficiency: A Commonly Overlooked Cause of Lung Disease. CMAJ : Canadian Medical Association journal = journal de l'Association medicale canadienne 2012, 184, 1365-1371

(172) Mirza, S.; Clay, R. D.; Koslow, M. A.; Scanlon, P. D. Copd Guidelines: A Review of the 2018 Gold Report. Mayo Clinic Proceedings 2018, 93, 1488-1502.

(173) Christiansen, A.; Davidsen, J. R.; Titlestad, I.; Vestbo, J.; Baumbach, J. A Systematic Review of Breath Analysis and Detection of Volatile Organic Compounds in Copd. Journal of Breath Research 2016, 10, 1-14.

(174) Besa, V.; Teschler, H.; Kurth, I.; Khan, A. M.; Zarogoulidis, P.; Baumbach, J. I.; Sommerwerck, U.; Freitag, L.; Darwiche, K. Exhaled Volatile Organic Compounds Discriminate Patients with Chronic Obstructive Pulmonary Disease from Healthy Subjects. Int J Chron Obstruct Pulmon Dis 2015, 10, 399-406.

(175) Hauschild, A. C.; Baumbach, J. I.; Baumbach, J. Integrated Statistical Learning of Metabolic Ion Mobility Spectrometry Profiles for Pulmonary Disease Identification. Genet Mol Res 2012, 11, 2733-2744.

(176) Westhoff, M.; Litterst, P.; Maddula, S.; Bödeker, B.; Baumbach, J. I. Statistical and Bioinformatical Methods to Differentiate Chronic Obstructive Pulmonary Disease (Copd) Including Lung Cancer from Healthy Control by Breath Analysis Using Ion Mobility Spectrometry. International Journal for Ion Mobility Spectrometry 2011, 14, 139-149.

(177) Cazzola, M.; Segreti, A.; Capuano, R.; Bergamini, A.; Martinelli, E.; Calzetta, L.; Rogliani, P.; Ciaprini, C.; Ora, J.; Paolesse, R. et al. Analysis of Exhaled Breath Fingerprints and Volatile Organic Compounds in Copd. COPD Research and Practice 2015, 1, 1-8.

(178) Fens, N.; Roldaan, A. C.; van der Schee, M. P.; Boksem, R. J.; Zwinderman, A. H.; Bel, E. H.; Sterk, P. J. External Validation of Exhaled Breath Profiling Using an Electronic Nose in the Discrimination of Asthma with Fixed Airways Obstruction and Chronic Obstructive Pulmonary Disease. Clin Exp Allergy 2011, 41, 1371-1378.

(179) Bregy, L.; Nussbaumer-Ochsner, Y.; Martinez-Lozano Sinues, P.; García-Gómez, D.; Suter, Y.; Gaisl, T.; Stebler, N.; Gaugg, M.; Kohler, M.; Zenobi, R. Real-Time Mass Spectrometric Identification of Metabolites Characteristic of Chronic Obstructive Pulmonary Disease in Exhaled Breath. Clinical Mass Spectrometry 2018, 7, 29-35.

(180) Martinez-Lozano Sinues, P.; Meier, L.; Berchtold, C.; Ivanov, M.; Sievi, N.; Camen, G.; Kohler, M.; Zenobi, R. Breath Analysis in Real Time by Mass Spectrometry in Chronic Obstructive Pulmonary Disease. Respiration 2014, 87, 301-310.

(181) Incalzi, R. A.; Pennazza, G.; Scarlata, S.; Santonico, M.; Petriaggi, M.; Chiurco, D.; Pedone, C.; D'Amico, A. Reproducibility and Respiratory Function Correlates of Exhaled Breath Fingerprint in Chronic Obstructive Pulmonary Disease. PLoS One 2012, 7, 1-8.

(182) O'Sullivan, B. P.; Freedman, S. D. Cystic Fibrosis. Lancet 2009, 373, 1891-1904.

(183) Goetz, D.; Ren, C. L. Review of Cystic Fibrosis. Pediatric Annals 2019, 48, E154-E161.

(184) Castellani, C.; Duff, A. J. A.; Bell, S. C.; Heijerman, H. G. M.; Munck, A.; Ratjen, F.; Sermet-Gaudelus, I.; Southern, K. W.; Barben, J.; Flume, P. A. et al. Ecfs Best Practice Guidelines: The 2018 Revision. Journal of Cystic Fibrosis 2018, 17, 153-178.
(185) Rosenfeld, M.; Ramsey, B. W.; Gibson, R. L. Pseudomonas Acquisition in Young Patients with Cystic Fibrosis: Pathophysiology, Diagnosis, and Management. Curr Opin Pulm Med 2003, 9, 492-497.

(186) Armstrong, D. S.; Hook, S. M.; Jamsen, K. M.; Nixon, G. M.; Carzino, R.; Carlin, J. B.; Robertson, C. F.; Grimwood, $\mathrm{K}$. Lower Airway Inflammation in Infants with Cystic Fibrosis Detected by Newborn Screening. Pediatr Pulmonol 2005, 40, 500-510.

(187) Hartl, D.; Gaggar, A.; Bruscia, E.; Hector, A.; Marcos, V.; Jung, A.; Greene, C.; McElvaney, G.; Mall, M.; Doring, G. Innate Immunity in Cystic Fibrosis Lung Disease. J Cyst Fibros 2012, 11, 363-382.

(188) Ahmed, W. M.; Lawal, O.; Nijsen, T. M.; Goodacre, R.; Fowler, S. J. Exhaled Volatile Organic Compounds of Infection: A Systematic Review. ACS Infect Dis 2017, 3, 695-710.

(189) Ratiu, I.-A.; Ligor, T.; Bocos-Bintintan, V.; Buszewski, B. Mass Spectrometric Techniques for the Analysis of Volatile Organic Compounds Emitted from Bacteria. Bioanalysis 2017, 9, 1069-1092.

(190) Kramer, R.; Sauer-Heilborn, A.; Welte, T.; Guzman, C. A.; Hofle, M. G.; Abraham, W. R. A Rapid Method for Breath Analysis in Cystic Fibrosis Patients. Eur J Clin Microbiol Infect Dis 2015, 34, 745-751.

(191) Shestivska, V.; Nemec, A.; Drevinek, P.; Sovova, K.; Dryahina, K.; Spanel, P. Quantification of Methyl Thiocyanate in the Headspace of Pseudomonas Aeruginosa Cultures and in the Breath of Cystic Fibrosis Patients by Selected Ion Flow Tube Mass Spectrometry. Rapid Communications in Mass Spectrometry 2011, 25, 24592467.

(192) Robroeks, C. M.; van Berkel, J. J.; Dallinga, J. W.; Jobsis, Q.; Zimmermann, L. J.; Hendriks, H. J.; Wouters, M. F.; van der Grinten, C. P.; van de Kant, K. D.; van Schooten, F. J. et al. Metabolomics of Volatile Organic Compounds in Cystic Fibrosis Patients and Controls. Pediatr Res 2010, 68, 75-80.

(193) Smith, D.; Sovova, K.; Dryahina, K.; Dousova, T.; Drevinek, P.; Spanel, P. Breath Concentration of Acetic Acid Vapour Is Elevated in Patients with Cystic Fibrosis. Journal of Breath Research 2016, 10, 1-6.

(194) Gaisl, T.; Bregy, L.; Stebler, N.; Gaugg, M. T.; Bruderer, T.; Garcia-Gomez, D.; Moeller, A.; Singer, F.; Schwarz, E. I.; Benden, C. et al. Real-Time Exhaled Breath Analysis in Patients with Cystic Fibrosis and Controls. Journal of Breath Research 2018, 12, 1-8.

(195) Brenner, D. J.; Hall, E. J. Computed Tomography — an Increasing Source of Radiation Exposure. New England Journal of Medicine 2007, 357, 2277-2284.

(196) Bach, P. B.; Mirkin, J. N.; Oliver, T. K.; Azzoli, C. G.; Berry, D. A.; Brawley, O. W.; Byers, T.; Colditz, G. A.; Gould, M. K.; Jett, J. R. et al. Benefits and Harms of Ct Screening for Lung Cancer: A Systematic Reviewbenefits and Harms of $\mathrm{Ct}$ Screening for Lung Cancer. JAMA 2012, 307, 2418-2429.

(197) Bajtarevic, A.; Ager, C.; Pienz, M.; Klieber, M.; Schwarz, K.; Ligor, M.; Ligor, T.; Filipiak, W.; Denz, H.; Fiegl, M. et al. Noninvasive Detection of Lung Cancer by Analysis of Exhaled Breath. BMC Cancer 2009, 9, 1-16.

(198) Feinberg, T.; Alkoby-Meshulam, L.; Herbig, J.; Cancilla, J. C.; Torrecilla, J. S.; Gai Mor, N.; Bar, J.; Ilouze, M.; Haick, H.; Peled, N. Cancerous Glucose Metabolism in Lung Cancer-Evidence from Exhaled Breath Analysis. Journal of Breath Research 2016, 10, 1-8.

(199) Wehinger, A.; Schmid, A.; Mechtcheriakov, S.; Ledochowski, M.; Grabmer, C.; Gastl, G. A.; Amann, A. Lung Cancer Detection by Proton Transfer Reaction MassSpectrometric Analysis of Human Breath Gas. International Journal of Mass Spectrometry 2007, 265, 4959.

(200) Handa, H.; Usuba, A.; Maddula, S.; Baumbach, J. I.; Mineshita, M.; Miyazawa, T. Exhaled Breath Analysis for 
Lung Cancer Detection Using Ion Mobility Spectrometry. PLoS One 2014, 9, 1-13.

(201) Westhoff, M.; Litterst, P.; Freitag, L.; Urfer, W.; Bader, S.; Baumbach, J. I. Ion Mobility Spectrometry for the Detection of Volatile Organic Compounds in Exhaled Breath of Patients with Lung Cancer: Results of a Pilot Study. Thorax 2009, 64, 744-748.

(202) Tirzite, M.; Bukovskis, M.; Strazda, G.; Jurka, N.; Taivans, I. Detection of Lung Cancer with Electronic Nose and Logistic Regression Analysis. Journal of Breath Research 2018, 13, 1-9.

(203) Hanna, G. B.; Boshier, P. R.; Markar, S. R.; Romano, A. Accuracy and Methodologic Challenges of Volatile Organic Compound-Based Exhaled Breath Tests for Cancer Diagnosis: A Systematic Review and Meta-Analysis. JAMA Oncol 2018, 5.

(204) Filipiak, W.; Mochalski, P.; Filipiak, A.; Ager, C.; Cumeras, R.; Davis, C. E.; Agapiou, A.; Unterkofler, K.; Troppmair, J. A Compendium of Volatile Organic Compounds (Vocs) Released by Human Cell Lines. Curr Med Chem 2016, 23, 2112-2131.

(205) Phillips, M.; Bauer, T. L.; Cataneo, R. N.; Lebauer, C.; Mundada, M.; Pass, H. I.; Ramakrishna, N.; Rom, W. N.; Vallières, E. Blinded Validation of Breath Biomarkers of Lung Cancer, a Potential Ancillary to Chest Ct Screening. PLOS ONE 2015, 10, 1-17.

(206) Klevens, R. M.; Edwards, J. R.; Richards, C. L.; Horan, T. C.; Gaynes, R. P.; Pollock, D. A.; Cardo, D. M. Estimating Health Care-Associated Infections and Deaths in U.S. Hospitals, 2002. Public Health Reports 2007, 122, 160-166.

(207) Restrepo, M. I.; Faverio, P.; Anzueto, A. Long-Term Prognosis in Community-Acquired Pneumonia. Current opinion in infectious diseases 2013, 26, 151-158.

(208) (NICE), N. I. f. H. a. C. E. "Pneumonia in Adults: Diagnosis and Management ", 2014.

(209) Pouline, M. v. O.; Pedro, P.; Ronny, S.; Paul, D.; Antonio, A.; Dennis, C. J. J. B.; Timothy, F.; Luis, C.; Marcus, J. S.; Stephen, J. F. et al. The Potential Role of Exhaled Breath Analysis in the Diagnostic Process of Pneumonia-a Systematic Review. Journal of Breath Research 2018, 12, $1-10$.

(210) Schnabel, R.; Fijten, R.; Smolinska, A.; Dallinga, J.; Boumans, M.-L.; Stobberingh, E.; Boots, A.; Roekaerts, P.; Bergmans, D.; van Schooten, F. J. Analysis of Volatile Organic Compounds in Exhaled Breath to Diagnose Ventilator-Associated Pneumonia. Scientific Reports 2015, 5, 1-10.

(211) Fowler, S. J.; Basanta-Sanchez, M.; Xu, Y.; Goodacre, R.; Dark, P. M. Surveillance for Lower Airway Pathogens in Mechanically Ventilated Patients by Metabolomic Analysis of Exhaled Breath: A Case-Control Study. Thorax 2015, 70, 320-325.

(212) van Oort, M. P.; de Bruin, S.; Weda, H.; Knobel, H. H.; Schultz, J. M.; Bos, D. L.; On Behalf of the, M. C. Exhaled Breath Metabolomics for the Diagnosis of Pneumonia in Intubated and Mechanically-Ventilated Intensive Care Unit (Icu)-Patients. International Journal of Molecular Sciences 2017, 18, 1-14.

(213) Zhu, J.; Bean, H. D.; Jiménez-Díaz, J.; Hill, J. E. Secondary Electrospray Ionization-Mass Spectrometry (SESI-MS) Breathprinting of Multiple Bacterial Lung Pathogens, a Mouse Model Study. Journal of Applied Physiology 2013, 114, 1544-1549.

(214) Jiangjiang, Z.; Heather, D. B.; Matthew, J. W.; Laurie, W. L.; Jane, E. H. Detecting Bacterial Lung Infections: In Vivo Evaluation of in Vitro Volatile Fingerprints. Journal of Breath Research 2013, 7, 1-12.

(215) Heather, D. B.; Jiangjiang, Z.; Jackson, C. S.; Jane, E. H. Identifying Methicillin-Resistant Staphylococcus Aureus (Mrsa) Lung Infections in Mice Via Breath Analysis Using Secondary Electrospray Ionization-Mass Spectrometry (SESI-MS). Journal of Breath Research 2014, 8, 1-6.
(216) Bean, H. D.; Jiménez-Díaz, J.; Zhu, J.; Hill, J. E. Breathprints of Model Murine Bacterial Lung Infections Are Linked with Immune Response. European Respiratory Journal 2015, 45, 181-190.

(217) Kapur, V. K.; Auckley, D. H.; Chowdhuri, S.; Kuhlmann, D. C.; Mehra, R.; Ramar, K.; Harrod, C. G. Clinical Practice Guideline for Diagnostic Testing for Adult Obstructive Sleep Apnea: An American Academy of Sleep Medicine Clinical Practice Guideline. Journal of clinical sleep medicine : JCSM : official publication of the American Academy of Sleep Medicine 2017, 13, 479-504.

(218) Kunos, L.; Bikov, A.; Lazar, Z.; Korosi, B. Z.; Benedek, P.; Losonczy, G.; Horvath, I. Evening and Morning Exhaled Volatile Compound Patterns Are Different in Obstructive Sleep Apnoea Assessed with Electronic Nose. Sleep Breath 2015, 19, 247-253.

(219) Dragonieri, S.; Porcelli, F.; Longobardi, F.; Carratu, P.; Aliani, M.; Ventura, V. A.; Tutino, M.; Quaranta, V. N.; Resta, O.; de Gennaro, G. An Electronic Nose in the Discrimination of Obese Patients with and without Obstructive Sleep Apnoea. Journal of Breath Research 2015, 9, 1-9.

(220) Benedek, P.; Lazar, Z.; Bikov, A.; Kunos, L.; Katona, G.; Horvath, I. Exhaled Biomarker Pattern Is Altered in Children with Obstructive Sleep Apnoea Syndrome. Int J Pediatr Otorhinolaryngol 2013, 77, 1244-1247.

(221) Greulich, T.; Hattesohl, A.; Grabisch, A.; Koepke, J.; Schmid, S.; Noeske, S.; Nell, C.; Wencker, M.; Jorres, R. A.; Vogelmeier, C. F. et al. Detection of Obstructive Sleep Apnoea by an Electronic Nose. Eur Respir J 2013, 42, 145155.

(222) Schwarz, E. I.; Engler, A.; Kohler, M. Exhaled Breath Analysis in Obstructive Sleep Apnea. Expert Rev Respir Med 2017, 11, 631-639.

(223) Tszyrsznic, W.; Borowiec, A.; Pawlowska, E.; Jazwiec, R.; Zochowska, D.; Bartlomiejczyk, I.; Zegarska, J.; Paczek, L.; Dadlez, M. Two Rapid Ultra Performance Liquid Chromatography/Tandem Mass Spectrometry (Uplc/Ms/Ms) Methods with Common Sample Pretreatment for Therapeutic Drug Monitoring of Immunosuppressants Compared to Immunoassay. Journal of Chromatography $B$ 2013, 928, 9-15.

(224) Esther, C. R.; Boucher, R. C.; Johnson, M. R.; Ansede, J. H.; Donn, K. H.; O’Riordan, T. G.; Ghio, A. J.; Hirsh, A. J. Airway Drug Pharmacokinetics Via Analysis of Exhaled Breath Condensate. Pulmonary pharmacology \& therapeutics 2014, 27, 76-82.

(225) Beck, O.; Sandqvist, S.; Eriksen, P.; Franck, J.; Palmskog, G. Determination of Methadone in Exhaled Breath Condensate by Liquid Chromatography-Tandem Mass Spectrometry. Journal of Analytical Toxicology 2011, 35, 129-133.

(226) Esther, C. R.; Boucher, R. C.; Johnson, M. R.; Ansede, J. H.; Donn, K. H.; O’Riordan, T. G.; Ghio, A. J.; Hirsh, A. J. Airway Drug Pharmacokinetics Via Analysis of Exhaled Breath Condensate. Pulmonary pharmacology \& therapeutics 2014, 27, 76-82.

(227) Beauchamp, J. Inhaled Today, Not Gone Tomorrow: Pharmacokinetics and Environmental Exposure of Volatiles in Exhaled Breath. Journal of Breath Research 2011, 5, 115.

(228) Berchtold, C.; Bosilkovska, M.; Daali, Y.; Walder, B.; Zenobi, R. Real-Time Monitoring of Exhaled Drugs by Mass Spectrometry. Mass Spectrometry Reviews 2013, 33, 394-413.

(229) Harrison, G. R.; Critchley, A. D. J.; Mayhew, C. A.; Thompson, J. M. Real-Time Breath Monitoring of Propofol and Its Volatile Metabolites During Surgery Using a Novel Mass Spectrometric Technique: A Feasibility Study. Br. J. Anaesth. 2003, 91, 797-799.

(230) Erhart, S.; Amann, A.; Haberlandt, E.; Edlinger, G.; Schmid, A.; Filipiak, W.; Schwarz, K.; Mochalski, P.; Rostasy, K.; Karall, D. et al. 3-Heptanone as a Potential 
New Marker for Valproic Acid Therapy. Journal of Breath Research 2009, 3, 1-6.

(231) Li, X.; Martinez-Lozano Sinues, P.; Dallmann, R.; Bregy, L.; Hollmén, M.; Proulx, S.; Brown, S. A.; Detmar, M.; Kohler, M.; Zenobi, R. Drug Pharmacokinetics Determined by Real-Time Analysis of Mouse Breath. Angewandte Chemie - International Edition 2015, 54, 7815-7818.

(232) Gamez, G.; Zhu, L.; Disko, A.; Chen, H.; Azov, V.; Chingin, K.; Krämer, G.; Zenobi, R. Real-Time, in Vivo Monitoring and Pharmacokinetics of Valproic Acid Via a Novel Biomarker in Exhaled Breath. Chemical Communications 2011, 47, 4884-4886.

(233) Beauchamp, J.; Kirsch, F.; Buettner, A. Real-Time Breath Gas Analysis for Pharmacokinetics: Monitoring Exhaled Breath by on-Line Proton-Transfer-Reaction Mass Spectrometry after Ingestion of Eucalyptol-Containing Capsules. Journal of Breath Research 2010, 4, 1-12.

(234) Martinez-Lozano Sinues, P.; Kohler, M.; Brown, S. A.; Zenobi, R.; Dallmann, R. Gauging Circadian Variation in Ketamine Metabolism by Real-Time Breath Analysis. Chem Commun 2017, 53, 2264-2267.

(235) Szymczak, W.; Rozman, J.; Hoellriegl, V.; Kistler, M.; Keller, S.; Peters, D. D.; Kneipp, M.; Schulz, H.; Hoeschen, C.; Klingenspor, M. et al. Online Breath Gas Analysis in Unrestrained Mice by Hs-PTR-MS. Mammalian genome : official journal of the International Mammalian Genome Society 2013, 25, 129-140.

(236) Kistler, M.; Muntean, A.; Höllriegl, V.; Matuschek, G.; Zimmermann, R.; Hoeschen, C.; de Angelis, M. H.; Rozman, J. A Systemic View on the Distribution of DietDerived Methanol and Hepatic Acetone in Mice. Journal of Breath Research 2017, 12, 1-10.

(237) Aprea, E.; Morisco, F.; Biasioli, F.; Vitaglione, P.; Cappellin, L.; Soukoulis, C.; Lembo, V.; Gasperi, F.; D'Argenio, G.; Fogliano, V. et al. Analysis of Breath by Proton Transfer Reaction Time of Flight Mass Spectrometry in Rats with Steatohepatitis Induced by HighFat Diet. Journal of Mass Spectrometry 2012, 47, 10981103.

(238) Martinez-Lozano Sinues, P.; Kohler, M.; Brown, S. A.; Zenobi, R.; Dallmann, R. Gauging Circadian Variation in Ketamine Metabolism by Real-Time Breath Analysis. Chemical Communications 2017, 53, 2264-2267.

(239) Beauchamp, J. Inhaled Today, Not Gone Tomorrow: Pharmacokinetics and Environmental Exposure of Volatiles in Exhaled Breath. J. Breath Res. 2011, 5, 037103.

(240) Beauchamp, J.; Kirsch, F.; Buettner, A. Real-Time Breath Gas Analysis for Pharmacokinetics: Monitoring Exhaled Breath by on-Line Proton-Transfer-Reaction Mass Spectrometry after Ingestion of Eucalyptol-Containing Capsules. Journal of Breath Research 2010, 4, 026006.

(241) Juric, A.; Fijacko, A.; Bakulic, L.; Oresic, T.; Gmajnicki, I. Evaluation of Breath Alcohol Analysers by Comparison of Breath and Blood Alcohol Concentrations. Arh Hig Rada Toksikol 2018, 69, 69-76.

(242) Mochalski, P.; King, J.; Klieber, M.; Unterkofler, K.; Hinterhuber, H.; Baumann, M.; Amann, A. Blood and Breath Levels of Selected Volatile Organic Compounds in Healthy Volunteers. Analyst 2013, 138, 2134-2145.

(243) O'Hara, M. E.; Clutton-Brock, T. H.; Green, S.; Mayhew, C. A. Endogenous Volatile Organic Compounds in Breath and Blood of Healthy Volunteers: Examining Breath Analysis as a Surrogate for Blood Measurements. Journal of Breath Research 2009, 3, 1-10.

(244) King, J.; Koc, H.; Unterkofler, K.; Mochalski, P.; Kupferthaler, A.; Teschl, G.; Teschl, S.; Hinterhuber, H.; Amann, A. Physiological Modeling of Isoprene Dynamics in Exhaled Breath. $J$ Theor Biol 2010, 267, 626-637.

(245) Miekisch, W.; Fuchs, P.; Kamysek, S.; Neumann, C.; Schubert, J. K. Assessment of Propofol Concentrations in Human Breath and Blood by Means of Hs-Spme-Gc-Ms. Clin Chim Acta 2008, 395, 32-37.
(246) Miekisch, W.; Schubert, J. K.; Vagts, D. A.; Geiger, K. Analysis of Volatile Disease Markers in Blood. Clin Chem 2001, 47, 1053-1060.

(247) Mochalski, P.; Al-Zoairy, R.; Niederwanger, A.; Unterkofler, K.; Amann, A. Quantitative Analysis of Volatile Organic Compounds Released and Consumed by Rat L6 Skeletal Muscle Cells in Vitro. Journal of Breath Research 2014, 8, 1-7.

(248) Cailleux, A.; Cogny, M.; Allain, P. Blood Isoprene Concentrations in Humans and in Some Animal Species. Biochem Med Metab Biol 1992, 47, 157-160.

(249) Taylor, S. G. a. A. A. a. S. Advances in Chemical Sensing Technologies for Vocs in Breath for Security/Threat Assessment, Illicit

Drug Detection, and Human Trafficking Activity. Journal of Breath Research 2018, 12, 1-18.

(250) Huo, R.; Agapiou, A.; Bocos-Bintintan, V.; Brown, L. J.; Burns, C.; Creaser, C. S.; Devenport, N. A.; Gao-Lau, B.; Guallar-Hoyas, C.; Hildebrand, L. et al. The Trapped Human Experiment. Journal of Breath Research 2011, 5, 112.

(251) Mochalski, P.; Unterkofler, K.; Teschl, G.; Amann, A. Potential of Volatile Organic Compounds as Markers of Entrapped Humans for Use in Urban Search-and-Rescue Operations. TrAC Trends in Analytical Chemistry 2015, 68, 88-106.

(252) Martinez-Lozano Sinues, P.; Tarokh, L.; Li, X.; Kohler, M.; Brown, S. A.; Zenobi, R.; Dallmann, R. Circadian Variation of the Human Metabolome Captured by Real-Time Breath Analysis. PLoS One 2014, 9, 1-16.

(253) Trefz, P.; Kamysek, S.; Fuchs, P.; Sukul, P.; Schubert, J. K.; Miekisch, W. Drug Detection in Breath: Non-Invasive Assessment of Illicit or Pharmaceutical Drugs. Journal of Breath Research 2017, 11, 1-14.

(254) Garcia-Gomez, D.; Gaisl, T.; Bregy, L.; Martinez-Lozano Sinues, P.; Kohler, M.; Zenobi, R. Secondary Electrospray Ionization Coupled to High-Resolution Mass Spectrometry Reveals Tryptophan Pathway Metabolites in Exhaled Human Breath. Chem Commun 2016, 52, 8526-8528.

(255) Vishinkin, R.; Haick, H. Nanoscale Sensor Technologies for Disease Detection Via Volatolomics. Small 2015, 11, 6142-6164.

(256) Zheng, X. Y.; Xu, Y. J.; Guan, W. J.; Lin, L. F. Regional, Age and Respiratory-Secretion-Specific Prevalence of Respiratory Viruses Associated with Asthma Exacerbation: A Literature Review. Arch Virol 2018, 163, 845-853.

(257) Bannier, M.; van de Kant, K. D. G.; Jobsis, Q.; Dompeling, E. Feasibility and Diagnostic Accuracy of an Electronic Nose in Children with Asthma and Cystic Fibrosis. Journal of Breath Research 2018, 13, 1-7.

(258) Klaassen, E. M.; van de Kant, K. D.; Jobsis, Q.; van Schayck, O. C.; Smolinska, A.; Dallinga, J. W.; van Schooten, F. J.; den Hartog, G. J.; de Jongste, J. C.; Rijkers, G. T. et al. Exhaled Biomarkers and Gene Expression at Preschool Age Improve Asthma Prediction at 6 Years of Age. Am J Respir Crit Care Med 2015, 191, 201-207.

(259) Moschino, L.; Zanconato, S.; Bozzetto, S.; Baraldi, E.; Carraro, S. Childhood Asthma Biomarkers: Present Knowledge and Future Steps. Paediatr Respir Rev 2015, 16, 205-212.

(260) Smolinska, A.; Klaassen, E. M.; Dallinga, J. W.; van de Kant, K. D.; Jobsis, Q.; Moonen, E. J.; van Schayck, O. C.; Dompeling, E.; van Schooten, F. J. Profiling of Volatile Organic Compounds in Exhaled Breath as a Strategy to Find Early Predictive Signatures of Asthma in Children. PLoS One 2014, 9, 1-10.

(261) van de Kant, K. D.; van Berkel, J. J.; Jobsis, Q.; Lima Passos, V.; Klaassen, E. M.; van der Sande, L.; van Schayck, O. C.; de Jongste, J. C.; van Schooten, F. J.; Derks, E. et al. Exhaled Breath Profiling in Diagnosing Wheezy Preschool Children. Eur Respir J 2013, 41, 183188. 
(262) van Vliet, D.; Smolinska, A.; Jobsis, Q.; Rosias, P.; Muris, J.; Dallinga, J.; Dompeling, E.; van Schooten, F. J. Can Exhaled Volatile Organic Compounds Predict Asthma Exacerbations in Children? Journal of Breath Research 2017, 11, 1-8.

(263) Van Vliet, D.; Smolinska, A.; Jobsis, Q.; Rosias, P. P.; Muris, J. W.; Dallinga, J. W.; van Schooten, F. J.; Dompeling, E. Association between Exhaled Inflammatory Markers and Asthma Control in Children. Journal of Breath Research 2016, 10, 1-9.

(264) Heaney, L. M.; Lindley, M. R. Translation of Exhaled Breath Volatile Analyses to Sport and Exercise Applications. Metabolomics 2017, 13, 1-9.

(265) King, J.; Kupferthaler, A.; Unterkofler, K.; Koc, H.; Teschl, S.; Teschl, G.; Miekisch, W.; Schubert, J.; Hinterhuber, H.; Amann, A. Isoprene and Acetone Concentration Profiles During Exercise on an Ergometer. Journal of Breath Research 2009, 3, 027006.

(266) Décombaz, J.; Grathwohl, D.; Pollien, P.; Schmitt, J. A. J.; Borrani, F.; Lecoultre, V. Effect of Short-Duration Lipid Supplementation on Fat Oxidation During Exercise and Cycling Performance. Applied Physiology, Nutrition, and Metabolism 2013, 38, 766-772.

(267) Meier, L.; Berchtold, C.; Schmid, S.; Zenobi, R. High Mass Resolution Breath Analysis Using Secondary Electrospray Ionization Mass Spectrometry Assisted by an Ion Funnel. Journal of Mass Spectrometry 2012, 47, 1571-1575.

(268) Costanzo, M. T.; Boock, J. J.; Kemperman, R. H. J.; Wei, M. S.; Beekman, C. R.; Yost, R. A. Portable Faims: Applications and Future Perspectives. International journal of mass spectrometry 2017, 422, 188-196.

(269) Cannabix Technologies Inc., B., BC, US. Cannabix Technologies. http://www.cannabixtechnologies.com/. 02.01.2019.

(270) Hound labs Inc. CA, U. Hound Labs. https://houndlabs.com/. 02.01.2019.

(271) Beck, O.; Leine, K.; Palmskog, G.; Franck, J. Amphetamines Detected in Exhaled Breath from Drug Addicts: A New Possible Method for Drugs-of-Abuse Testing. Journal of Analytical Toxicology 2010, 34, 233237.

(272) Beck, O.; Sandqvist, S.; Eriksen, P.; Franck, J.; Palmskog, G. Method for Determination of Methadone in Exhaled Breath Collected from Subjects Undergoing Methadone Maintenance Treatment. Journal of Chromatography B 2010, 878, 2255-2259.

(273) Olof, B.; Niclas, S.; Sören, S.; Johan, F. Detection of Drugs of Abuse in Exhaled Breath Using a Device for Rapid Collection: Comparison with Plasma, Urine and SelfReporting in 47 Drug Users. Journal of Breath Research 2013, 7, 1-10.

(274) Keller, B. O.; Suj, J.; Young, A. B.; Whittal, R. M. Interferences and Contaminants Encountered in Modern Mass Spectrometry. Analytica Chimica Acta 2008, 627, 7181.

(275) Sinues, P. M. L.; Kohler, M.; Zenobi, R. Monitoring Diurnal Changes in Exhaled Human Breath. Analytical Chemistry 2013, 85, 369-373.

(276) Sukul, P.; Oertel, P.; Kamysek, S.; Trefz, P. Oral or Nasal Breathing? Real-Time Effects of Switching Sampling Route onto Exhaled Voc Concentrations. Journal of Breath Research 2017, 11, 1-18.

(277) Sukul, P.; Schubert, J. K.; Oertel, P.; Kamysek, S.; Taunk, K.; Trefz, P.; Miekisch, W. Fev Manoeuvre Induced Changes in Breath Voc Compositions: An Unconventional View on Lung Function Tests. Scientific Reports 2016, 6, $1-12$.

(278) Sukul, P.; Schubert, J. K.; Kamysek, S.; Trefz, P.; Miekisch, W. Applied Upper-Airway Resistance Instantly Affects Breath Components: A Unique Insight into Pulmonary Medicine. Journal of Breath Research 2017, 11, $1-12$.
(279) Sukul, P.; Schubert, J. K.; Trefz, P.; Miekisch, W. Natural Menstrual Rhythm and Oral Contraception Diversely Affect Exhaled Breath Compositions. Scientific Reports 2018, 8, $1-10$.

(280) Smolinska, A.; Tedjo, D. I.; Blanchet, L.; Bodelier, A.; Pierik, M. J.; Masclee, A. A. M.; Dallinga, J.; Savelkoul, P. H. M.; Jonkers, D.; Penders, J. et al. Volatile Metabolites in Breath Strongly Correlate with Gut Microbiome in $\mathrm{Cd}$ Patients. Analytica Chimica Acta 2018, 1025, 1-11.

(281) Luo, J.; Schumacher, M.; Scherer, A.; Sanoudou, D.; Megherbi, D.; Davison, T.; Shi, T.; Tong, W.; Shi, L.; Hong, H. et al. A Comparison of Batch Effect Removal Methods for Enhancement of Prediction Performance Using Maqc-Ii Microarray Gene Expression Data. Pharmacogenomics Journal 2010, 10, 278-291.

(282) Nygaard, V.; Rodland, E. A.; Hovig, E. Methods That Remove Batch Effects While Retaining Group Differences May Lead to Exaggerated Confidence in Downstream Analyses. Biostatistics 2016, 17, 29-39.

(283) Higgins, J. P. T.; Green, S.; Cochrane Collaboration. Cochrane Handbook for Systematic Reviews of Interventions; Wiley-Blackwell: Chichester, England ; Hoboken, NJ, 2008.

(284) Head, M. L.; Holman, L.; Lanfear, R.; Kahn, A. T.; Jennions, M. D. The Extent and Consequences of PHacking in Science. Plos Biology 2015, 13, 1-15.

(285) Baxter, M. G.; Burwell, R. D. Promoting Transparency and Reproducibility in Behavioral Neuroscience: Publishing Replications, Registered Reports, and Null Results. Behav Neurosci 2017, 131, 275-276.

(286) Majchrzak, T.; Wojnowski, W.; Lubinska-Szczygeł, M.; Różańska, A.; Namieśnik, J.; Dymerski, T. PTR-MS and Gc-Ms as Complementary Techniques for Analysis of Volatiles: A Tutorial Review. Analytica Chimica Acta 2018, 1035, 1-13.

(287) Španěl, P.; Dryahina, K.; Smith, D. A General Method for the Calculation of Absolute Trace Gas Concentrations in Air and Breath from Selected Ion Flow Tube Mass Spectrometry Data. International Journal of Mass Spectrometry 2006, 249-250, 230-239.

(288) Španěl, P.; Smith, D. Quantitative Selected Ion Flow Tube Mass Spectrometry: The Influence of Ionic Diffusion and Mass Discrimination. Journal of the American Society for Mass Spectrometry 2001, 12, 863-872.

(289) Daniel B. Milligan, †; Gregory J. Francis, †; Barry J. Prince, $\ddagger$ and; Murray J. McEwan*, $\$$. Demonstration of Selected Ion Flow Tube Ms Detection in the Parts Per Trillion Range. Analytical Chemistry 2007, 79, 2537-2540.

(290) Dummer, J. F.; Storer, M. K.; Hu, W.-P.; Swanney, M. P.; Milne, G. J.; Frampton, C. M.; Scotter, J. M.; Prisk, G. K.; Epton, M. J. Accurate, Reproducible Measurement of Acetone Concentration in Breath Using Selected Ion Flow Tube-Mass Spectrometry. Journal of breath research 2010 , 4, 1-8.

(291) Graus, M.; Müller, M.; Hansel, A. High Resolution Ptr-Tof: Quantification and Formula Confirmation of Voc in Real Time. Journal of the American Society for Mass Spectrometry 2010, 21, 1037-1044.

(292) Cappellin, L.; Karl, T.; Probst, M.; Ismailova, O.; Winkler, P. M.; Soukoulis, C.; Aprea, E.; Märk, T. D.; Gasperi, F.; Biasioli, F. On Quantitative Determination of Volatile Organic Compound Concentrations Using Proton Transfer Reaction Time-of-Flight Mass Spectrometry. Environmental Science \& Technology 2012, 46, 2283-2290.

(293) Hansel, A.; Jordan, A.; Warneke, C.; Holzinger, R.; Wisthaler, A.; Lindinger, W. Proton-Transfer-Reaction Mass Spectrometry (PTR-MS): On-Line Monitoring of Volatile Organic Compounds at Volume Mixing Ratios of a Few Pptv. Plasma Sources Science and Technology 1999, 8, 332-336.

(294) Lindinger, W.; Hansel, A. Analysis of Trace Gases at Ppb Levels by Proton Transfer Reaction Mass Spectrometry 
(PTR-MS). Plasma Sources Science and Technology 1997, 6, 111-117.

(295) Lindinger, W.; Hansel, A.; Jordan, A. On-Line Monitoring of Volatile Organic Compounds at Pptv Levels by Means of Proton-Transfer-Reaction Mass Spectrometry (PTR-MS) Medical Applications, Food Control and Environmental Research. International Journal of Mass Spectrometry and Ion Processes 1998, 173, 191-241.

(296) Uppal, K.; Walker, D. I.; Liu, K.; Li, S. Z.; Go, Y. M.; Jones, D. P. Computational Metabolomics: A Framework for the Million Metabolome. Chemical Research in Toxicology 2016, 29, 1956-1975.

(297) Madsen, R.; Lundstedt, T.; Trygg, J. Chemometrics in Metabolomics-a Review in Human Disease Diagnosis. Analytica Chimica Acta 2010, 659, 23-33.

(298) Yamamoto, H.; Yamaji, H.; Abe, Y.; Harada, K.; Waluyo, D.; Fukusaki, E.; Kondo, A.; Ohno, H.; Fukuda, H. Dimensionality Reduction for Metabolome Data Using Pca, Pls, Opls, and Rfda with Differential Penalties to Latent Variables. Chemometrics and Intelligent Laboratory Systems 2009, 98, 136-142.

(299) Johnstone, I. M.; Titterington, D. M. Statistical Challenges of High-Dimensional Data. Philosophical Transactions of the Royal Society a-Mathematical Physical and Engineering Sciences 2009, 367, 4237-4253.

(300) Li, F.; Wang, J. X.; Nie, L.; Zhang, W. W. In Metabolomics, 2012.

(301) Kusonmano, K.; Vongsangnak, W.; Chumnanpuen, P. In Translational Biomedical Informatics: A Precision Medicine Perspective; Shen, B.;Tang, H.;Jiang, X., Eds.; Springer Singapore: Singapore, 2016.

(302) Bartel, J.; Krumsiek, J.; Theis, F. J. Statistical Methods for the Analysis of High-Throughput Metabolomics Data. Computational and Structural Biotechnology Journal 2013, 4, 1-9.

(303) Bödeker, B.; Davies, A. N.; Maddula, S.; Baumbach, J. I. Biomarker Validation-Room Air Variation During Human Breath Investigations. International Journal for Ion Mobility Spectrometry 2010, 13, 177-184.

(304) Malo, N.; Hanley, J. A.; Carlile, G.; Liu, J.; Pelletier, J.; Thomas, D.; Nadon, R. Experimental Design and Statistical Methods for Improved Hit Detection in High-Throughput Screening. Journal of Biomolecular Screening 2010, 15, 990-1000.

(305) Nakagawa, S. A Farewell to Bonferroni: The Problems of Low Statistical Power and Publication Bias. Behavioral Ecology 2004, 15, 1044-1045.

(306) Ioannidis, J. P. Why Most Published Research Findings Are False. PLoS Med 2005, 2, 0696-0701.

(307) Colhoun, H. M.; McKeigue, P. M.; Davey Smith, G. Problems of Reporting Genetic Associations with Complex Outcomes. Lancet 2003, 361, 865-872.

(308) Schymanski, E. L.; Jeon, J.; Gulde, R.; Fenner, K.; Ruff, M.; Singer, H. P.; Hollender, J. Identifying Small Molecules Via High Resolution Mass Spectrometry: Communicating Confidence. Environmental Science \& Technology 2014, 48, 2097-2098.

(309) Stein, S. E. Estimating Probabilities of Correct Identification from Results of Mass Spectral Library Searches. Journal of the American Society for Mass Spectrometry 1994, 5, 316-323.

(310) Španěl, P.; Spesyvyi, A.; Smith, D. Electrostatic Switching and Selection of $\mathrm{H}_{30}+, \mathrm{No}^{+}$, and $\mathrm{O} 2+\bullet$ Reagent Ions for Selected Ion Flow-Drift Tube Mass Spectrometric Analyses of Air and Breath. Analytical Chemistry 2019, 91, 53805388.

(311) Wishart, D. S.; Tzur, D.; Knox, C.; Eisner, R.; Guo, A. C.; Young, N.; Cheng, D.; Jewell, K.; Arndt, D.; Sawhney, S. et al. Hmdb: The Human Metabolome Database. Nucleic acids research 2007, 35, D521-D526.

(312) Wishart, D. S.; Feunang, Y. D.; Marcu, A.; Guo, A. C.; Liang, K.; Vázquez-Fresno, R.; Sajed, T.; Johnson, D.; Li,
C.; Karu, N. et al. Hmdb 4.0: The Human Metabolome Database for 2018. Nucleic acids research 2018, 46, D608D617.

(313) Smith, C. A.; Maille, G. O.; Want, E. J.; Qin, C.; Trauger, S. A.; Brandon, T. R.; Custodio, D. E.; Abagyan, R.; Siuzdak, G. Metlin: A Metabolite Mass Spectral Database. Therapeutic Drug Monitoring 2005, 27, 747-751.

(314) Guijas, C.; Montenegro-Burke, J. R.; Domingo-Almenara, X.; Palermo, A.; Warth, B.; Hermann, G.; Koellensperger, G.; Huan, T.; Uritboonthai, W.; Aisporna, A. E. et al. Metlin: A Technology Platform for Identifying Knowns and Unknowns. Analytical Chemistry 2018, 90, 3156-3164.

(315) Technology, N. I. o. S. a. Nist Chemistry Webbook. http://webbook.nist.gov. 01.05.2019.

(316) Ltd, H. Mzcloud Database. www.mzcloud.org. 01.05.2019.

(317) Lemfack, M. C.; Gohlke, B.-O.; Toguem, S. M. T.; Preissner, S.; Piechulla, B.; Preissner, R. Mvoc 2.0: A Database of Microbial Volatiles. Nucleic acids research 2018, 46, D1261-D1265.

(318) Chemspider. Chemspider Chemical Database. http://www.chemspider.com/. 01.01.2019.

(319) Kim, S.; Thiessen, P. A.; Bolton, E. E.; Chen, J.; Fu, G.; Gindulyte, A.; Han, L.; He, J.; He, S.; Shoemaker, B. A. et al. Pubchem Substance and Compound Databases. Nucleic acids research 2016, 44, D1202-D1213.

(320) Vinaixa, M.; Schymanski, E. L.; Neumann, S.; Navarro, M.; Salek, R. M.; Yanes, O. Mass Spectral Databases for Lc/Ms- and Gc/Ms-Based Metabolomics: State of the Field and Future Prospects. TrAC Trends in Analytical Chemistry 2016, 78, 23-35.

(321) Böcker, S. Searching Molecular Structure Databases Using Tandem Ms Data: Are We There Yet? Current Opinion in Chemical Biology 2017, 36, 1-6.

(322) Siuzdak, G. Metlin: A Metabolite Mass Spectral Database. https://metlin.scripps.edu/. 01.05.2019.

(323) Kanehisa, M.; Goto, S. Kegg: Kyoto Encyclopedia of Genes and Genomes. Nucleic Acids Research 2000, 28, $27-$ 30.

(324) Snyder, D. T.; Pulliam, C. J.; Ouyang, Z.; Cooks, R. G. Miniature and Fieldable Mass Spectrometers: Recent Advances. Analytical Chemistry 2016, 88, 2-29.

(325) Riter, L. S.; Laughlin, B. C.; Nikolaev, E.; Cooks, R. G. Direct Analysis of Volatile Organic Compounds in Human Breath Using a Miniaturized Cylindrical Ion Trap Mass Spectrometer with a Membrane Inlet. Rapid Communications in Mass Spectrometry 2002, 16, 23702373.

\section{Biographies}

Tobias Bruderer is a postdoctoral fellow at the ETH Zurich and the University Children's Hospital Zurich. He obtained his Ph.D. in Analytical Chemistry with focus on high-resolution mass spectrometry, bioanalysis and metabolomics at the University of Geneva in 2016. His main research interests are the analysis of volatile organic compounds in medicine and environmental toxicology.

Thomas Gaisl obtained his M.D. at the Medical University of Innsbruck, Austria and then moved to Zurich where he obtained his doctorate in 2014 under the supervision of Prof. Kohler at the University of Zurich, Switzerland. He is currently a postdoctoral researcher at the University of Zurich 
with his research focus on breath-analysis, respiratory diseases and clinical trials.

Martin T. Gaugg is a Ph.D. candidate in the group of Prof. Zenobi at ETH Zurich. He was awarded his B.Sc. in Chemistry at Leopold-Franzens-University Innsbruck in 2013 as best of the graduating class and continued his education at ETH Zürich, where he completed his M.Sc. in Chemistry under the Excellence Scholarship and Opportunity Programme in 2015. Already during his studies, Gaugg joined the laboratory of Renato Zenobi, where he developed automated data-processing algorithms for on-line breath analysis. He is currently completing his Ph.D. studies at ETH in collaboration with the University Hospital Zurich, investigating new biomarkers for respiratory diseases using breath analysis.

Nora Nowak is a $\mathrm{Ph}$. D. candidate in the group of Professor Renato Zenobi at the Swiss Federal Institute of Technology (ETH) Zurich. Her research interests include real-time metabolomics in exhaled breath using secondary electrospray ionization. She received her B.Sc. and M.Sc. in chemistry from Friedrich-Schiller University in Jena in Germany. During her Master's she moved to France for a semester at Ecole Nationale Supérieure de Chimie de Montpellier and she completed her master thesis at Plateforme de Protéomique Clinique Montpellier in the group of Prof. S. Lehmann.

Bettina Streckenbach is a Ph.D. candidate in the laboratory of Prof. Zenobi at ETH Zurich. After her Bachelor studies in Biosciences at the University of Potsdam, she received her M.Sc. in Biochemistry from the University of Konstanz. During her master thesis in a joint project of Professor M. Morbidelli and R. Zenobi (both ETH Zurich) she approached biotechnological questions in monoclonal antibody production using mass spectrometry. She currently works on ambient ionization mass spectrometry for breath analysis focusing on its clinical applicability.

Simona Müller received her BSc (2015) and MSc (2017) degree in chemistry from the University of Zurich. She then joined Prof. Dr. Renato Zenobi's research group as a graduate student in the field of breath analysis with SESI-MS.

Alexander Moeller is Professor for paediatric pulmonology at the University Children's Hospital Zurich, Switzerland. He graduated at the University of Zurich 1994 and obtained the title of doctor in medicine in the year 1996. He specialized in paediatrics (2001), paediatric pulmonology (2003) and paediatric sleep science (2008). Since 2007 he is head of the Division of respiratory medicine at the University Children's Hospital Zurich. His specific research interest is the non-invasive assessment of airway inflammation in children, specifically the clinical value of exhaled nitric oxide in young children and breath and exhaled breath condensate analysis by means of mass spectrometry.

Malcolm Kohler is Professor and Chair of Respiratory Medicine at the University of Zurich and Clinical Director of the Department of Pulmonology of the University Hospital of Zurich, Switzerland. He initiated and directs a $\mathrm{PhD}$ program in clinical science at the University of Zurich. His research focuses on the pathophysiology and treatment of chronic respiratory disorders such as obstructive sleep apnea and chronic obstructive pulmonary disease. Together with Professor Zenobi he leads the Zurich Exhalomics flagship project, a research consortium which explores the metabolome of breath with diverse real-time technologies. Professor Kohler has contributed $>250$ scientific articles to the field of respiratory medicine and some of this work has been acknowledged with national and international awards.

Renato Zenobi is Professor of Analytical Chemistry at the Swiss Federal Institute of Technology (ETH) Zurich. After a Ph.D. at Stanford University and postdoctoral appointments at the University of Pittsburgh and at the University of Michigan, he started his independent career in 1992 as a Werner Fellow at EPF Lausanne. He joined ETH as an assistant professor in 1995 and was promoted to full professor in 2000. Zenobi has made important contributions to the understanding of the ion formation mechanism in matrix-assisted laser desorption/ionization (MALDI) mass spectrometry, to the development of analytical tools for the nanoscale, in particular tip-enhanced Raman spectroscopy (TERS), and to exhaled breath analysis using ambient ionization methods. 